Anna Dall'Acqua*, Chun-Chi Lin, and Paola Pozzi

\title{
Elastic flow of networks: long-time existence result
}

https://doi.org/10.1515/geofl-2019-0005

Received January 12, 2019; accepted November 28, 2019

Abstract: We provide a long-time existence and sub-convergence result for the elastic flow of a three-network in $\mathbb{R}^{n}$ under some mild topological assumptions. The evolution is such that the sum of the elastic energies of the three curves plus their weighted lengths decrease in time. Natural boundary conditions are considered at the boundary of the curves and at the triple junction.

Keywords: elastic flow, networks, junctions, long-time existence

MSC: primary 35K52, 53C44; secondary 35K61, 35K41

\section{Introduction}

We consider the long-time evolution for the elastic flow of a three-network in $\mathbb{R}^{n}(n \geq 2)$ as depicted in Figure 1, that is with three fixed boundary points $P_{1}, P_{2}, P_{3}$ and one moving triple junction. That is, a threepointed curved star with a star-center that may move in time.

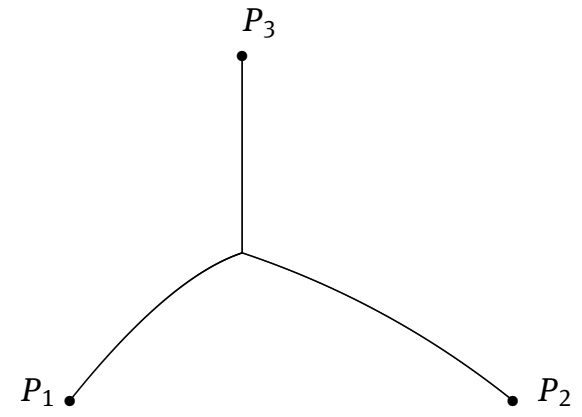

Figure 1: The model

We study and give a long time existence result for elastic motion with a penalization term on the length and some extra topological conditions that prevents the appearance of some pathological cases: for instance, the triple junction should not be allowed to collapse on one of the boundary points $P_{i}$.

As far as we know, research on elastic flow of networks is still at its beginning stage in mathematics. A lot of literature is focused on the case where the motion occurs by mean curvature flow (also called curve shortening flow, a second order flow that decreases length of the curves), see for instance the survey paper [20]. A

^Corresponding Author: Anna Dall’Acqua: Institut für Analysis, Universität Ulm, Germany,

E-mail: anna.dallacqua@uni-ulm.de

Chun-Chi Lin: Department of Mathematics, National Taiwan Normal University, Taipei, 116 Taiwan,

E-mail: chunlin@math.ntnu.edu.tw

Paola Pozzi: Fakultät für Mathematik, Universität Duisburg-Essen, Germany, E-mail: paola.pozzi@uni-due.de 
short time existence result for the elastic flow of a network of planar curves has been given recently in [13] while the stationary case (in the special case of so-called 'Theta' networks) has been considered in [7]. Elastic flow with junctions is considered numerically in [1]: in particular an appropriate variational formulation and two types of junction conditions, the so called $C^{0}$ respectively $C^{1}$ boundary conditions, are discussed. In that work the authors concentrated in the derivation of the Euler-Lagrange equations and did many numerical simulations.

Networks and flow of networks arise naturally in the study of multiphase systems and of the dynamics of their interfaces, see for instance [14, 18]. Elastic networks appear in some investigation in mechanical engineering or material sciences related to polymer gels, fiber or protein networks, e.g., [3], [15]. In these physical systems, junctions between elastic beams play an important role in determining mechanical properties, e.g., rigidity or deformability.

Before stating our main result, we introduce briefly the set up of our work and recall some well known facts. The elastic energy of a smooth regular curve (an immersion) $f: \bar{I} \rightarrow \mathbb{R}^{n}, n \geq 2, I=(0,1)$ is given by

$$
\mathcal{E}(f)=\frac{1}{2} \int_{I}|\vec{\kappa}|^{2} d s
$$

where $d s=\left|\partial_{x} f\right| d x$ is the arc-length element and $\vec{\kappa}$ is the curvature vector of the curve. Defining $\partial_{s}=$ $\left|\partial_{x} f\right|^{-1} \partial_{x} f$, then $\vec{\kappa}=\partial_{S}^{2} f$. The length of a curve $f$ is given by

$$
\mathcal{L}(f)=\int_{I} d s .
$$

For $\lambda \geq 0$ let

$$
\varepsilon_{\lambda}(f)=\mathcal{E}(f)+\lambda \mathcal{L}(f) .
$$

This is the energy that we consider: the length of the curve is allowed to change in time but its growth is penalized according to the weight $\lambda$.

Now consider three smooth regular curves $f_{i}: \bar{I} \rightarrow \mathbb{R}^{n}, I=(0,1), i=1,2,3$, such that

1. The end-points are fixed:

$$
f_{1}(1)=P_{1}, f_{2}(1)=P_{2}, f_{3}(1)=P_{3},
$$

with given distinct points $P_{i}, i=1,2,3$, in $\mathbb{R}^{n}$ (recall Figure ??). Of course, there is a plane that contains these three points.

2. The curves start at the same point

$$
f_{1}(0)=f_{2}(0)=f_{3}(0) \quad \text { (concurrency condition). }
$$

In the following we call $\Gamma=\left\{f_{1}, f_{2}, f_{3}\right\}$ a three-pointed star network or simply network.

For $\lambda=\left(\lambda_{1}, \lambda_{2}, \lambda_{3}\right) \in \mathbb{R}_{+}^{3}$, the energy of the network $\Gamma=\left\{f_{1}, f_{2}, f_{3}\right\}$ is given by

$$
\varepsilon_{\lambda}(\Gamma)=\sum_{i=1}^{3} \varepsilon_{\lambda_{i}}\left(f_{i}\right) .
$$

Here and in the following we agree that $\varepsilon(\Gamma):=\varepsilon_{0}(\Gamma)$.

We let the network $\Gamma$ evolve in time according to an $L^{2}$-gradient flow for the energy $\varepsilon_{\lambda}$. Natural boundary conditions are imposed on the three curves (more details are given in Section 2). Our main result is the following:

Theorem 1.1. Let $\Gamma_{0}=\left\{f_{1,0}, f_{2,0}, f_{3,0}\right\}$ be a network of regular smooth curves in $\mathbb{R}^{n}$ parametrized by constant speed such that:

$$
\begin{aligned}
f_{1,0}(1) & =P_{1}, f_{2,0}(1)=P_{2}, f_{3,0}(1)=P_{3}, \\
f_{1,0}(0) & =f_{2,0}(0)=f_{3,0}(0), \\
\vec{\kappa}_{i, 0}(x) & =0 \text { for } x=0,1 \text { and } i=1,2,3
\end{aligned}
$$


as well as

$$
\left.\sum_{i=1}^{3}\left(\nabla_{s} \vec{\kappa}_{i, 0}-\lambda_{i} \partial_{s} f_{i, 0}\right)\right|_{x=0}=0 .
$$

Moreover let $\Gamma_{0}$ satisfy appropriate compatibility conditions (specified in Remark C.3), and be such that at the triple junction at least two curves form a strictly positive angle. Then the following holds:

(i) Long-time existence result: the equations

$$
\partial_{t} f_{i}-\left\langle\partial_{t} f_{i}, \partial_{s} f_{i}\right\rangle \partial_{s} f_{i}=-\nabla_{s}^{2} \vec{\kappa}_{i}-\frac{1}{2}\left|\vec{\kappa}_{i}\right|^{2} \vec{\kappa}_{i}+\lambda_{i} \vec{k}_{i} \text { on }(0, T) \times I \text { for } i=1,2,3,
$$

with boundary conditions

$$
\left\{\begin{array}{lr}
f_{i}(t, 1)=P_{i}, & \text { for all } t \in[0, T), i=1,2,3, \\
\vec{\kappa}_{i}(t, 1)=0=\vec{\kappa}_{i}(t, 0) & \text { for all } t \in[0, T), i=1,2,3, \\
f_{1}(t, 0)=f_{2}(t, 0)=f_{3}(t, 0) & \text { for all } t \in[0, T), \\
\text { and } \sum_{i=1}^{3}\left(\nabla_{s} \vec{\kappa}_{i}(t, 0)-\lambda_{i} \partial_{s} f_{i}(t, 0)\right)=0 & \text { for all } t \in[0, T),
\end{array}\right.
$$

and initial datum

$$
\Gamma(t=0)=\left\{f_{1}(0, \cdot), f_{2}(0, \cdot), f_{3}(0, \cdot)\right\}=\Gamma_{0}, \text { in } I
$$

admit a smooth global solution in time, provided that, along the flow, the lengths $\mathcal{L}\left(f_{i}\right)$ of the three curves are uniformly bounded from below and that the dimension of the space spanned by the unit tangents $\partial_{s} f_{i}, i=1,2,3$, at the triple junction is bigger than or equal to two (see Assumption 6.1).

(ii) Sub-convergence result: under the mentioned hypothesis, and provided none of the lengths of the curves goes to zero as $t \rightarrow \infty$, it is possible to find a sequence of time $t_{i} \rightarrow \infty$ such that the networks $\Gamma\left(t_{i}\right)$ smoothly sub-converge, after an appropriate reparametrization, to a critical point for the energy $\mathcal{E}_{\lambda}(\Gamma)$ and subject to the boundary conditions given in (1.6) and (1.7).

Remark 1.2. In Remarks 6.3, 6.4 and 6.6 below several generalizations of this result are discussed.

Smooth solution means that the three parametrization of the three curves are smooth functions in the time and space variable. The extension of the long-time existence result to the case $\lambda_{i} \geq 0$ is discussed in Remark 6.3 below.

Note that the above theorem must be understood in a geometrical sense: that is the existence of a global parametrization of the flow is meant up to reparametrization. So our result states that we are able to find a global in time smooth motion of the network, provided two topological constraints are fulfilled during the flow: namely that the lengths of the curves are uniformly bounded from below and that the the curves never entirely "collapse" to a configuration where all tangents vectors are parallel at the triple junctions. In our proof the topological constraints occurs naturally as follows: the bound from below on the lengths of the curves is needed to apply interpolation inequalities (cf. for instance Lemma 3.10 and Lemma 3.11 below); that the dimension of the space spanned by the unit tangents at the triple junction should always be bigger or equal to two arise when we express the tangential components at the boundary in terms of geometric quantities (cf. Remark 5.1 and Remark 5.2 below.) Not surprisingly it arises also in the proof of short-time existence of the flow provided in [6] and [13]. At the moment we have no means to control these topological constraints: whether and how this could be done is subject to future studies (see also Remark 6.5 below).

To achieve our goal, we will consider in place of (1.8) equations of type

$$
\partial_{t} f_{i}=-\nabla_{s}^{2} \vec{\kappa}_{i}-\frac{1}{2}\left|\vec{\kappa}_{i}\right|^{2} \vec{\kappa}_{i}+\lambda_{i} \vec{\kappa}_{i}+\varphi_{i} \partial_{s} f_{i} \text { on }(0, T) \times I \text { for } i=1,2,3,
$$


where $\varphi_{i}$ are smooth functions. Note that the presence of the tangential components is necessary in order for the flow to fulfill the topological constraint that the curves stay "glued" at the triple junction (concurrency condition), with the latter being able to move freely in time. A proper choice of tangential component is necessary and is discussed in details in Section 2.3 and in Section C.

Our strategy can be summarized as follows: starting from a short-time existence result (see Section 2.3) we reparametrize the flow in such a way that for each curve the maps $\varphi_{i}$ linearly interpolate their values between the boundary points. For this choice of parametrizations we consider the long-time behavior of the network, and show that if the flow does not exist globally then we obtain a contradiction. This is achieved by obtaining uniform bounds for the curvature and its derivatives, together with a control on the arc-length, up to the maximal time of existence $0<T<\infty$. With these estimates we are able to extend the flow smoothly up to $T$ and then restart the flow, contradicting the maximality of $T$.

In its essence the strategy of our proof is not different from our previous works on long-time existence for open elastic curves in $\mathbb{R}^{n}$ (see [4], [5], [8], [9], which in turn rely on ideas of [10] and [22]): we use inequalities of Gagliardo-Nirenberg type, exploit the boundary conditions to reduce the order of some boundary terms, and rely heavily on interpolation estimates presented in [5]. However, the treatment of the tangential components is completely new and far from trivial. In particular the "algebra" for the maps $\varphi_{i}$ (that is how their derivatives in time and space behave with respect to the order of the studied PDEs, see Remark 4.3 for more details) must be thoroughly understood. Furthermore, an accurate choice of the "right" vector field (specifically $\vec{\phi}$ in Lemma 3.2) for which uniform bounds are derived is absolutely crucial for any of the presented arguments to work. Finally, because of the interaction of the three curves proofs become increasingly technical and lengthy, and several new lemmas are derived in order to make our arguments more concise and more transparent.

The paper is organized as follows: after introducing the notation and motivating the definition of the flow in Section 2, we collect several preliminary estimates and interpolation estimates in Section 3. The treatment of the boundary term is given in Section 4, whereas the influence of the tangential components at the boundary is discussed in Section 5. Finally, in Section 6 we prove the main Theorem 1.1. The proof of the latter is divided in several steps: we have an initial step, where first bounds on the curvature vectors are derived. In the second step, we show the starting procedure of an induction argument: it is at this point that a proper choice of $\varphi_{i}$ starts playing an important role. After the somewhat cumbersome induction step, where uniform estimates for the derivative of the curvature vectors are derived, we are finally able to conclude long-time existence by the contradiction procedure mentioned above. To ease the presentation many technical proofs are collected in the Appendix where we also cite the results on short-time existence that are needed for our present work.

During the time between the submission of this work and its revision an interesting preprint [12] appeared where long-time existence for planar networks moving by elastic energy (as in this work) is studied but with the difference that weaker function spaces are used. Moreover, in [21] the authors provide a long-time existence result for a different flow of the elastic energy in the case of planar networks with curves of fixed lengths. The relation between the setting and the results presented here and those depicted in [21] are discussed there.

Acknowledgements: This project has been funded by the Deutsche Forschungsgemeinschaft (DFG, German Research Foundation)- Projektnummer: 404870139, and Ministry of Science and Technology, Taiwan (MoST 107-2923-M-003 -001 -MY3). Moreover, we would like to thank the anonymous referee for his/her precious feedback. 


\section{Set up and notation}

\subsection{First variation and natural boundary conditions}

First of all recall, that for sufficiently smooth $f, \phi: \bar{I} \rightarrow \mathbb{R}^{n}$, with $f$ a regular curve, the first variation of the length is given by

$$
\left.\frac{d}{d \varepsilon} \mathcal{L}(f+\varepsilon \phi)\right|_{\varepsilon=0}=\left.\frac{d}{d \varepsilon} \int_{I}\left|\partial_{x}(f+\varepsilon \phi)\right| d x\right|_{\varepsilon=0}=\left.\left\langle\partial_{S} f, \phi\right\rangle\right|_{\partial I}-\int_{I}\langle\vec{\kappa}, \phi\rangle d s,
$$

while the first variation of elastic energy (1.1) (see [8, Proof of Lemma A1]) is

$$
\begin{aligned}
\left.\frac{d}{d \varepsilon} \varepsilon(f+\varepsilon \phi)\right|_{\varepsilon=0} & =\left.\frac{d}{d \varepsilon} \int_{I}\left|\vec{\kappa}_{f+\varepsilon \phi}\right|^{2}\left|\partial_{x}(f+\varepsilon \phi)\right| d x\right|_{\varepsilon=0} \\
& =\left.\left\langle\partial_{S} \phi, \vec{\kappa}\right\rangle\right|_{\partial I}-\left.\left\langle\phi, \nabla_{s} \vec{\kappa}+\frac{1}{2}|\vec{\kappa}|^{2} \partial_{S} f\right\rangle\right|_{\partial I}+\int_{I}\left\langle\nabla_{S}^{2} \vec{\kappa}+\frac{1}{2}|\vec{\kappa}|^{2} \vec{\kappa}, \phi\right\rangle d s .
\end{aligned}
$$

Here $\nabla_{s}$ is an operator that on a smooth vector field $\phi$ acts as follows

$$
\nabla_{s} \phi=\partial_{s} \phi-\left\langle\partial_{s} \phi, \partial_{s} f\right\rangle \partial_{s} f,
$$

i.e. it is the normal projection of $\partial_{s} \phi$.

Let consider a variation $\Gamma_{t \eta}$ of the network $\Gamma$ given by $t \in(-\delta, \delta)$ and sufficiently smooth vector fields $\left\{\eta_{1}, \eta_{2}, \eta_{3}\right\}, \eta_{i}: I \rightarrow \mathbb{R}^{n}$, such that

$$
\eta_{i}(1)=0 \text { for } i=1,2,3 \text { and } \eta_{i}(0)=\eta_{j}(0) \text { for } i, j=1,2,3,
$$

so that $\Gamma_{t \eta}=\left\{f_{1}+t \eta_{1}, f_{2}+t \eta_{2}, f_{3}+t \eta_{3}\right\}$ is still a three-pointed star network.

Then by (2.1) and (2.2) we find

$$
\begin{gathered}
\left.\frac{d}{d t}\right|_{t=0} \varepsilon_{\lambda}\left(\Gamma_{t \eta}\right)=\sum_{i=1}^{3}\left(\left.\left\langle\partial_{s} \eta_{i}, \vec{\kappa}_{i}\right\rangle\right|_{\partial I}-\left.\left\langle\eta_{i}, \nabla_{s} \vec{\kappa}_{i}+\frac{1}{2}\left|\vec{\kappa}_{i}\right|^{2} \partial_{s} f_{i}\right\rangle\right|_{\partial I}+\left.\lambda_{i}\left\langle\partial_{s} f_{i}, \eta_{i}\right\rangle\right|_{\partial I}\right. \\
\left.+\int_{I}\left\langle\nabla_{s}^{2} \vec{\kappa}_{i}+\frac{1}{2}\left|\vec{\kappa}_{i}\right|^{2} \vec{\kappa}_{i}, \eta_{i}\right\rangle d s-\lambda_{i} \int_{I}\left\langle\vec{\kappa}_{i}, \eta_{i}\right\rangle d s\right) .
\end{gathered}
$$

Notice that here and in the rest of the work for simplicity of notation we simply write $d s$ instead of $d s^{i}$ and also in the derivatives we simply write $\nabla_{s}$ instead of the correct $\nabla_{s^{i}}$.

Choosing first test functions with compact support we see that each critical point has to satisfy

$$
\nabla_{s}^{2} \vec{\kappa}_{i}+\frac{1}{2}\left|\vec{\kappa}_{i}\right|^{2} \vec{\kappa}_{i}-\lambda_{i} \vec{\kappa}_{i}=0 \text {, on }(0,1),
$$

$i=1,2,3$. Moreover, at $x=1$

$$
\left.\sum_{i=1}^{3}\left\langle\partial_{s} \eta_{i}, \vec{\kappa}_{i}\right\rangle\right|_{x=1}=0
$$

for any test function $\eta_{i}$ and hence that

$$
\vec{\kappa}_{i}(1)=0 \text { for } i=1,2,3 \text {. }
$$

Instead, at $x=0$ we find

$$
\left.\sum_{i=1}^{3}\left(\left\langle\partial_{s} \eta_{i}, \vec{\kappa}_{i}\right\rangle-\left\langle\eta_{i}, \nabla_{s} \vec{\kappa}_{i}+\left(\frac{1}{2}\left|\vec{\kappa}_{i}\right|^{2}-\lambda_{i}\right) \partial_{s} f_{i}\right\rangle\right)\right|_{x=0}=0 .
$$


This implies

$$
\vec{\kappa}_{i}(0)=0 \text { for } i=1,2,3,
$$

and together with $\eta_{1}(0)=\eta_{2}(0)=\eta_{3}(0)$ that

$$
\left.\sum_{i=1}^{3}\left(\nabla_{s} \vec{\kappa}_{i}-\lambda_{i} \partial_{s} f_{i}\right)\right|_{x=0}=0 .
$$

\subsection{The flow}

As mentioned in the introduction we take an $L^{2}$-flow for the energy $\varepsilon_{\lambda}$ with the condition that the network keeps its topological properties along the flow. For this one needs a tangential component. The problem we consider is then (1.10) with $\varphi_{i}, i=1,2,3$, smooth functions (whose role and definition is discussed below), with boundary conditions given in (1.9) and initial value

$$
\Gamma(t=0)=\left\{f_{1}(0, \cdot), f_{2}(0, \cdot), f_{3}(0, \cdot)\right\}=\Gamma_{0} \text {, in } I .
$$

For the latter we ask that $\Gamma_{0}=\left\{f_{1,0}, f_{2,0}, f_{3,0}\right\}$ be a network of regular smooth curves parametrized by constant speed, satisfying (1.6), (1.7) and being such that at the triple junction at least two curves form a strictly positive angle (the latter condition is necessary for our short-time existence result given in Section C). Moreover the initial network needs to satisfy a set of compatibility conditions. These are required to ensure that the solution of the parabolic problem is smooth up to the initial time $t=0$. We ask therefore that $f_{i, 0}$, $i=1,2,3$ satisfy compatibility condition of any order according to Remark C.3: indeed, with this choice and together with a very specific choice of tangential components $\varphi_{i}:=\varphi_{i}^{\star}$ as defined in (C1) we can start the flow smoothly (see Appendix $\mathrm{C}$ for the short-time existence result).

\subsection{Short time existence and reparametrization}

Our starting point is the short time existence result Theorem C.1, stating that given an initial network $\Gamma_{0}$ of smooth curves satisfying (1.6), (1.7) and suitable compatibility conditions (cf. Appendix C), then there exists an interval of time where our problem (1.10) (with $\varphi_{i}:=\varphi_{i}^{\star}$ as defined in (C1)) admits a smooth regular solution, meaning that $f_{i} \in C^{\infty}([0, T) \times[0,1]), i=1,2,3$, are regular parametrizations. A proof of this result is provided in [6]. A short-time existence for planar curves, in appropriate Hölder spaces can be found in [13].

Before we proceed some comments are in order, in particular more information must be given on the role/choice of the tangential components $\varphi_{i}$ in (1.10). First of all, notice that to construct a short-time existence solution for (1.8) (together with the chosen initial and boundary conditions), one typically proceeds by 1) introducing a suitable choice of tangential components $\varphi_{i}$ (in order to factor out the degeneracies due to the geometric invariances; in our case we take $\varphi_{i}:=\varphi_{i}^{\star}$ as defined in (C1)), 2) applying a linearization procedure and Solonnikov-theory (see [23]), 3) employing a fixed point argument to show short-time existence for the systems of non-linear equations under consideration (see [2]). In particular we see that at a first sight any tangential component $\varphi_{i}$ "destroys the geometric nature" of the equations (1.8). This is, however, not entirely true. It is well known, that tangential components can be modified by a reparametrization and that indeed all geometrical quantities (tangents, curvature vectors, length, etc.) are invariant under reparametrization. That, for the geometric motion, $\varphi_{i}$ plays no role in the interior of each curves becomes evident also during the computations performed in this paper. The role of the tangential components $\varphi_{i}$ becomes tangible only at the boundary of each curve, when enforcing the concurrency condition at the triple junction (see (1.4)) and influencing variation of the length of each curve (cf. also Remark 3.3 below). On the other hand even at the boundary the tangential components are determined by geometric quantities (see Remark 5.1, in particular (5.3) below). So the "freedom of choice" in the tangential components is in principle only allowed in the interior of the curve, where, as we have already stated, the geometric quantities do not "register" it. Needles to 
say, we want to avoid tangential components, hence parametrizations, that destroy the regularity properties of the flow.

For the long-time existence proof it is important to have a good control of the tangential components also in the interior of the curves. To that end we reparametrize our short-time solution $f=\left(f_{1}, f_{2}, f_{3}\right)$ from Theorem C.1 in such a way that the new tangential component $\varphi_{i}^{G}$ interpolates linearly between the boundary data of $\varphi_{i}^{\star}$ at $x=0$ and $x=1$ (where $\varphi_{i}^{\star}(t, 1)=0$, since at $x=1$ the velocities vanish due to the boundary conditions). This transformation employes the fact that the tangential components are geometrical at the boundary points and hence independent of the parametrization (see Remark 5.1). To this end, we choose a family of smooth diffeomorphisms $\phi_{i}(t, \cdot): I \rightarrow I, i=1,2,3$, such that $\phi_{i}(t, x)=x$ for $(t, x) \in\left[0, T_{1}\right) \times \partial I$, for some $0<T_{1} \leq T$, and such that for

$$
\tilde{f}_{i}(t, x):=f_{i}\left(t, \phi_{i}(t, x)\right)
$$

and

$$
\begin{aligned}
\varphi_{i}^{G}(t, x): & =\varphi_{i}^{\star}(t, 0)\left(1-\frac{1}{\mathcal{L}\left(f_{i}(t)\right)} \int_{0}^{x}\left|\partial_{x} f_{i}\left(t, \phi_{i}(t, x)\right)\right| \partial_{x} \phi_{i}(t, x) d x\right) \\
& =\varphi_{i}^{\star}(t, 0)\left(1-\frac{1}{\mathcal{L}\left(\tilde{f}_{i}(t)\right)} \int_{0}^{x}\left|\partial_{x} \tilde{f}_{i}(t, \xi)\right| d \xi\right) .
\end{aligned}
$$

there holds

$$
\begin{aligned}
\partial_{t} \phi_{i}(t, x) & =\left(\varphi_{i}^{G}(t, x)-\varphi_{i}^{\star}\left(t, \phi_{i}(t, x)\right)\right) /\left|\partial_{x} f_{i}\left(t, \phi_{i}(t, x)\right)\right|, \quad \text { for } x \in I, \\
\phi_{i}(0, x) & =x .
\end{aligned}
$$

Existence and uniqueness follow from Lemma C.4. A straightforward computation gives then that

$$
\begin{aligned}
\partial_{t} \tilde{f}_{i}(t, x) & =\partial_{t} f_{i}\left(t, \phi_{i}(t, x)\right)+\partial_{x} f_{i}\left(t, \phi_{i}(t, x)\right) \partial_{t} \phi_{i}(t, x) \\
& =\left[\partial_{t} f_{i}-\left\langle\partial_{t} f_{i}, \partial_{s} f_{i}\right\rangle \partial_{s} f_{i}\right]\left(t, \phi_{i}(t, x)\right)+\varphi_{i}^{G}(t, x) \partial_{s} f_{i}\left(t, \phi_{i}(t, x)\right) \\
& =\left[-\nabla_{s}^{2} \overrightarrow{\tilde{\kappa}}_{i}-\frac{1}{2}\left|\overrightarrow{\tilde{\kappa}}_{i}\right|^{2} \overrightarrow{\tilde{\kappa}}_{i}+\lambda_{i} \overrightarrow{\tilde{\kappa}}_{i}+\varphi_{i}^{G} \partial_{s} \tilde{f}_{i}\right](t, x) .
\end{aligned}
$$

In other words we can reparametrize the flow in such a way that the tangential component interpolates linearly its boundary values. At the same time problem (1.8) is satisfied on $\left[0, T_{1}\right)$. As we will see in the proof of the long time existence, this will be of great help in many estimates. Also note that

$$
\varphi_{i}^{\star}(t, x)=\varphi_{i}^{G}(t, x) \text {, for }(t, x) \in[0, T) \times \partial I,
$$

as well as

$$
\partial_{t}^{m} \varphi_{i}^{\star}(t, x)=\partial_{t}^{m} \varphi_{i}^{G}(t, x), \text { for }(t, x) \in[0, T) \times \partial I, \text { and } m \in \mathbb{N}
$$

so that $\tilde{f}_{i}$ fulfills all the same boundary conditions as $f_{i}, i=1,2,3$. Again, let us stress the fact that by Remark 5.1, in particular (5.3) below, the tangential components $\varphi_{i}^{G}=\varphi_{i}^{\star}$ at the boundary are terms that can be given in terms of geometric quantities (curvature vectors, tangents, etc.), which of course do not change under reparametrizations of the flow. In particular $\varphi_{i}^{G}$ can be written in terms of geometric quantities for the network $\tilde{f}$ (cf. (5.3) and Remark 5.1) and we can write

$$
\varphi_{i}^{G}(t, x)=\varphi_{i}^{G}(t, 0)\left(1-\frac{1}{\mathcal{L}\left(\tilde{f}_{i}(t)\right)} \int_{0}^{x}\left|\partial_{x} \tilde{f}_{i}(t, \xi)\right| d \xi\right) .
$$

This is to say that we have achieved an expression that is independent of the diffeomorphisms $\phi_{i}$ used above in the derivation.

We refer to (2.5), (2.6), with boundary conditions (1.9) (with $f$ replaced by $\tilde{f}$ ) as the geometric problem. The 
observations made so far yield that given an initial data satisfying the assumptions of Theorem 1.1, then there exists a short-time solution to the geometric problem. For the sake of simplicity we drop the notation $\tilde{f}$.

Summarizing, from now on we consider the geometric problem (1.10), (1.9), whereby $\varphi_{i}\left(:=\varphi_{i}^{G}\right)$ linearly interpolates between its boundary values, i.e it satisfies (2.6) (see also (6.6)). It is for this problem that we want to obtain a long-time existence result.

\section{Preliminaries}

First of all we state a simple fact that will be used repeatedly in the computations that follow: for $\vec{\phi}$ any smooth normal field along $f$ and $h$ a scalar map we have that for any $m \in \mathbb{N}$ (using (2.3))

$$
\begin{array}{ll}
\nabla_{s}(h \vec{\phi})=\left(\partial_{s} h\right) \vec{\phi}+h \nabla_{s} \vec{\phi}, & \nabla_{s}^{m}(h \vec{\phi})=\sum_{r=0}^{m}\left(\begin{array}{c}
m \\
r
\end{array}\right) \partial_{s}^{m-r} h \nabla_{s}^{r} \vec{\phi} \\
\nabla_{t}(h \vec{\phi})=\left(\partial_{t} h\right) \vec{\phi}+h \nabla_{t} \vec{\phi}, & \nabla_{t}^{m}(h \vec{\phi})=\sum_{r=0}^{m}\left(\begin{array}{c}
m \\
r
\end{array}\right) \partial_{t}^{m-r} h \nabla_{t}^{r} \vec{\phi} \\
\nabla_{t}\left(h \partial_{s} f\right)=h \nabla_{t}\left(\partial_{s} f\right), &
\end{array}
$$

where $\nabla_{t} \phi=\partial_{t} \phi-\left\langle\partial_{t} \phi, \partial_{s} f\right\rangle \partial_{s} f$.

Lemma 3.1 (Evolution of geometric quantities). Let $f:[0, T) \times I \rightarrow \mathbb{R}^{n}$ be a smooth solution of $\partial_{t} f=\vec{V}+\varphi \tau$ for $t \in(0, T)$ with $\vec{V}$ the normal velocity. Given $\vec{\phi}$ any smooth normal field along $f$, the following formulas hold.

$$
\begin{aligned}
\partial_{t}(d s) & =\left(\partial_{s} \varphi-\langle\vec{\kappa}, \vec{V}\rangle\right) d s \\
\partial_{t} \partial_{s}-\partial_{s} \partial_{t} & =\left(\langle\vec{\kappa}, \vec{V}\rangle-\partial_{s} \varphi\right) \partial_{s} \\
\partial_{t} \tau & =\nabla_{s} \vec{V}+\varphi \vec{\kappa} \\
\partial_{t} \vec{\phi} & =\nabla_{t} \vec{\phi}-\left\langle\nabla_{s} \vec{V}+\varphi \vec{\kappa}, \vec{\phi}\right\rangle \tau \\
\partial_{t} \vec{\kappa} & =\partial_{s} \nabla_{s} \vec{V}+\langle\vec{\kappa}, \vec{V}\rangle \vec{\kappa}+\varphi \partial_{s} \vec{\kappa} \\
\nabla_{t} \vec{\kappa} & =\nabla_{s}^{2} \vec{V}+\langle\vec{\kappa}, \vec{V}\rangle \vec{\kappa}+\varphi \nabla_{s} \vec{\kappa} \\
\left(\nabla_{t} \nabla_{s}-\nabla_{s} \nabla_{t}\right) \vec{\phi} & =\left(\langle\vec{\kappa}, \vec{V}\rangle-\partial_{s} \varphi\right) \nabla_{s} \vec{\phi}+\left[\langle\vec{\kappa}, \vec{\phi}\rangle \nabla_{s} \vec{V}-\left\langle\nabla_{s} \vec{V}, \vec{\phi}\right\rangle \vec{\kappa}\right] .
\end{aligned}
$$

Proof. The proof follows by straightforward computation: see for instance [8, Lemma 2.1] and [10].

\subsubsection{Decrease of the energy along the flow.}

As a first application of the above lemma we show that the energy decreases along the flow. Let $\Gamma(t)=$ $\left\{f_{1}(t, \cdot), f_{2}(t, \cdot), f_{3}(t, \cdot)\right\}$ be a three-pointed star network moving according the elastic flow as considered in Section 2.2. Then by (1.2), (3.4) and (3.9) we find

$$
\begin{aligned}
\frac{d}{d t} \sum_{i=1}^{3} \mathcal{E}_{\lambda}\left(f_{i}\right)=\sum_{i=1}^{3} \int_{I}\left\langle\vec{\kappa}_{i}, \nabla_{s}^{2} \vec{V}_{i}+\left\langle\vec{\kappa}_{i}, \vec{V}_{i}\right\rangle \vec{\kappa}_{i}+\varphi_{i} \nabla_{s} \vec{\kappa}_{i}\right\rangle d s \\
\left.+\sum_{i=1}^{3} \int_{I}\left(\frac{1}{2}\left|\vec{\kappa}_{i}\right|^{2}+\lambda_{i}\right)\left(\partial_{s} \varphi_{i}-\left\langle\vec{\kappa}_{i}, \vec{V}_{i}\right\rangle\right)\right\rangle d s .
\end{aligned}
$$

Integrating by parts and using that the curvature is zero at both boundary points

$$
\frac{d}{d t} \sum_{i=1}^{3} \varepsilon_{\lambda}\left(f_{i}\right)=-\sum_{i=1}^{3} \int_{I}\left\langle\nabla_{s} \vec{\kappa}_{i}, \nabla_{s} \vec{V}_{i}\right\rangle d s+\sum_{i=1}^{3} \int_{I}\left\langle\vec{\kappa}_{i},\left\langle\vec{\kappa}_{i}, \vec{V}_{i}\right\rangle \vec{\kappa}_{i}+\varphi_{i} \nabla_{s} \vec{\kappa}_{i}\right\rangle d s
$$




$$
\begin{aligned}
& +\left.\sum_{i=1}^{3} \lambda_{i} \varphi_{i}\right|_{\partial I}-\sum_{i=1}^{3} \int_{I} \varphi_{i}\left\langle\vec{\kappa}_{i}, \nabla_{s} \vec{\kappa}_{i}\right\rangle d s-\sum_{i=1}^{3} \int_{I}\left(\frac{1}{2}\left|\vec{\kappa}_{i}\right|^{2}+\lambda_{i}\right)\left\langle\vec{\kappa}_{i}, \vec{V}_{i}\right\rangle d s \\
=- & \left.\sum_{i=1}^{3}\left\langle\nabla_{s} \vec{\kappa}_{i}, \vec{V}_{i}\right\rangle\right|_{\partial I}+\sum_{i=1}^{3} \int_{I}\left\langle\nabla_{s}^{2} \vec{\kappa}_{i}, \vec{V}_{i}\right\rangle d s \\
& +\left.\sum_{i=1}^{3} \lambda_{i} \varphi_{i}\right|_{\partial I}+\sum_{i=1}^{3} \int_{I}\left(\frac{1}{2}\left|\vec{k}_{i}\right|^{2}-\lambda_{i}\right)\left\langle\vec{\kappa}_{i}, \vec{V}_{i}\right\rangle d s \\
= & -\left.\sum_{i=1}^{3}\left\langle\nabla_{s} \vec{\kappa}_{i}-\lambda_{i} \partial_{s} f_{i}, \vec{V}_{i}+\varphi_{i} \partial_{s} f_{i}\right\rangle\right|_{\partial I}+\sum_{i=1}^{3} \int_{I}\left\langle-\partial_{t} f_{i}+\varphi_{i} \partial_{s} f_{i}, \vec{V}_{i}\right\rangle d s \\
= & -\left.\sum_{i=1}^{3}\left\langle\nabla_{s} \vec{\kappa}_{i}-\lambda_{i} \partial_{s} f_{i}, \partial_{t} f_{i}\right\rangle\right|_{\partial I}-\sum_{i=1}^{3} \int_{I}\left|\partial_{t} f_{i}-\varphi_{i} \partial_{s} f_{i}\right|^{2} d s,
\end{aligned}
$$

since $\vec{V}_{i}=\partial_{t} f_{i}-\varphi_{i} \partial_{s} f_{i}$.

As $f(t, x=1)$ is fixed in time there is no contribution by the boundary terms at $x=1$. At $x=0$ one uses that $\partial_{t} f_{i}=\partial_{t} f_{j}$. Under natural boundary conditions at zero the boundary term also vanishes and we find that the energy is indeed decreasing.

Lemma 3.2 (Crucial lemma). Suppose $\partial_{t} f=\vec{V}+\varphi \tau$ on $(0, T) \times I$. Let $\vec{\phi}$ be a normal vector field along $f$ and $Y=\nabla_{t} \vec{\phi}+\nabla_{s}^{4} \vec{\phi}$. Then

$$
\begin{aligned}
\frac{d}{d t} \frac{1}{2} \int_{I}|\vec{\phi}|^{2} d s+\int_{I}\left|\nabla_{s}^{2} \vec{\phi}\right|^{2} d s=-\left[\left\langle\vec{\phi}, \nabla_{s}^{3} \vec{\phi}\right\rangle\right]_{0}^{1}+\left[\left\langle\nabla_{s} \vec{\phi}, \nabla_{s}^{2} \vec{\phi}\right\rangle\right]_{0}^{1} \\
+\int_{I}\left\langle Y+\frac{1}{2} \vec{\phi} \varphi_{s}, \vec{\phi}\right\rangle d s-\frac{1}{2} \int_{I}|\vec{\phi}|^{2}\langle\vec{\kappa}, \vec{V}\rangle d s,
\end{aligned}
$$

Proof. See [8, Lemma 2.3] for a similar statement. The claim follows using (3.4) and integration by parts.

Remark 3.3. It turns out that the influence of the tangential part $\varphi$ is dectectable only at the end-points of the considered curve (this is in accordance with the fact that geometric quantities are independent of parametrization). Suppose that the curve $f$ moving according to the evolution law $\partial_{t} f=\vec{V}+\varphi \tau$ is fixed at the end-point $x=1$ (thus we think of $f$ as one of the three curves of the three-pointed curved star). We have that $\varphi(t, 1)=0$ (here the velocity is zero!) for all $t$ : then using (3.4) we observe that

$$
-\varphi(t, 0)=\varphi(t, 1)-\varphi(t, 0)=\int_{I}\left(\left|f_{x}\right|\right)_{t} d x+\int_{I}\langle\vec{\kappa}, \vec{V}\rangle d s .
$$

In other words at the moving point (think here at the triple junction) we infer

$$
\varphi(t, 0)=-\frac{d}{d t} \mathcal{L}(f)-\int_{I}\langle\vec{\kappa}, \vec{V}\rangle d s .
$$

In particular using the expression $V=-\nabla_{S}^{2} \vec{\kappa}-\frac{1}{2}|\vec{\kappa}|^{2} \vec{\kappa}+\lambda \vec{\kappa}$, integration by parts and the boundary conditions $\vec{\kappa}(0)=\vec{\kappa}(1)=0$ we obtain

$$
\frac{d}{d t} \mathcal{L}(f)+\varphi(t, 0)+\int_{I}\left|\nabla_{s} \vec{\kappa}\right|^{2} d s+\lambda \int_{I}|\vec{\kappa}|^{2} d s=\frac{1}{2} \int_{I}|\vec{\kappa}|^{4} d s .
$$

It is not surprising that the length plays a role, since $\varphi(t, 0)$ determines how the curve grows or shrinks.

As in [10, Lem.2.3] and [8, Sec.3] we denote by the product $\vec{\phi}_{1} \star \vec{\phi}_{2} \star \ldots \star \vec{\phi}_{k}$ the product of $k$ normal vector fields $\vec{\phi}_{i}(i=1, \ldots, k)$ defined as $\left\langle\vec{\phi}_{1}, \vec{\phi}_{2}\right\rangle \cdot . . \cdot\left\langle\vec{\phi}_{k-2}, \vec{\phi}_{k-1}\right\rangle \vec{\phi}_{k}$ if $k$ is odd and as $\left\langle\vec{\phi}_{1}, \vec{\phi}_{2}\right\rangle \cdot . . \cdot\left\langle\vec{\phi}_{k-1}, \vec{\phi}_{k}\right\rangle$, if $k$ 
is even. The expression $P_{b}^{a, c}(\vec{\kappa})$ stands for any linear combination of terms of the type

$$
\left(\nabla_{s}\right)^{i_{1}} \vec{\kappa} \star \ldots \star\left(\nabla_{s}\right)^{i_{b}} \vec{\kappa} \text { with } i_{1}+\ldots+i_{b}=a \text { and } \max i_{j} \leq c
$$

with universal, constant coefficients. Notice that $a$ gives the total number of derivatives, $b$ denotes the number of factors and $c$ gives a bound on the highest number of derivatives falling on one factor. With a slight abuse of notation, $\left|P_{b}^{a, c}(\vec{\phi})\right|$ denotes any linear combination with non-negative coefficients of terms of type

$$
\left|\nabla_{s}^{i_{1}} \vec{\phi}\right| \cdot\left|\nabla_{s}^{i_{2}} \vec{\phi}\right| \cdot \ldots \cdot\left|\nabla_{s}^{i_{b}} \vec{\phi}\right| \text { with } i_{1}+\cdots+i_{b}=a \text { and } \max i_{j} \leq c .
$$

Observe that for odd $b \in \mathbb{N}$ we have $\nabla_{s} P_{b}^{a, c}(\vec{\phi})=P_{b}^{a+1, c+1}(\vec{\phi})$. For sums we write

$$
\sum_{[[a, b]] \leq[[A, B]]} P_{b \leq C}^{a, c}(\vec{\kappa}):=\sum_{a=0}^{A} \sum_{b=1}^{2 A+B-2 a} \sum_{c=0}^{C} P_{b}^{a, c}(\vec{\kappa}) .
$$

Similarly we set $\sum_{[[a, b]] \leq[[A, B]]}\left|P_{b}^{a, c}(\vec{\phi})\right|:=\sum_{a=0}^{A} \sum_{b=1}^{2 A+B-2 a} \sum_{c=0}^{C}\left|P_{b}^{a, c}(\vec{\phi})\right|$. For our convenience and motivated by the interpolation inequalities below, we say that the order of $\sum_{[[a, b]] \leq[[A, B]]} P_{b}^{a, c}(\vec{\kappa})$ is equal to $A+B / 2$.

With this notation we can state the following results.

Lemma 3.4. We have the identities

$$
\begin{aligned}
& \partial_{s} \vec{\kappa}=\nabla_{s} \vec{\kappa}-|\vec{\kappa}|^{2} \tau, \\
& \partial_{s}^{m} \vec{\kappa}=\nabla_{s}^{m} \vec{\kappa}+\tau \sum_{\substack{[a, b]] \leq[[m-1,2]] \\
c \leq m-1, b \text { even }}} P_{b}^{a, c}(\vec{\kappa})+\sum_{\begin{array}{c}
{\left[\begin{array}{c}
[a, b]] \leq[[m-2,3]] \\
c \leq m-2
\end{array}\right. \text { odd }} \\
\text { of }
\end{array}} P_{b}^{a, c}(\vec{\kappa}) \quad \text { for } m \geq 2 .
\end{aligned}
$$

Proof. The proof can be found for instance in [8, Lemma 4.5]. The first claim is obtained directly using that

$$
\partial_{s} \vec{\kappa}=\nabla_{s} \vec{\kappa}+\left\langle\partial_{s} \vec{\kappa}, \tau\right\rangle \tau=\nabla_{s} \vec{\kappa}-|\vec{\kappa}|^{2} \tau .
$$

The second claim follows by induction.

The previous Lemma will help us to transform estimates obtained for $\nabla_{s}^{m} \vec{\kappa}$ into estimates for the full derivative $\partial_{s}^{m} \vec{\kappa}$. The reason why we work with the operator $\nabla_{s}$ (instead of the full derivative $\partial_{s}$ ) is that it naturally appears in the evolution equation and it is therefore the most natural choice to work with.

Lemma 3.5. Suppose $\partial_{t} f=-\nabla_{s}^{2} \vec{\kappa}+\lambda \vec{\kappa}-\frac{1}{2}|\vec{k}|^{2} \vec{\kappa}+\varphi \tau$, where $\lambda=\lambda(t)$. Then for $m \in \mathbb{N}_{0}$ we have

$$
\nabla_{t} \nabla_{s}^{m} \vec{\kappa}+\nabla_{S}^{4} \nabla_{s}^{m} \vec{\kappa}=P_{3}^{m+2, m+2}(\vec{\kappa})+\lambda\left(\nabla_{S}^{m+2} \vec{\kappa}+P_{3}^{m, m}(\vec{\kappa})\right)+P_{5}^{m, m}(\vec{\kappa})+\varphi \nabla_{s}^{m+1} \vec{\kappa} .
$$

If $\lambda$ is a given fixed constant then we also write

$$
\nabla_{t} \nabla_{s}^{m} \vec{\kappa}+\nabla_{s}^{4} \nabla_{s}^{m} \vec{\kappa}=\varphi \nabla_{s}^{m+1} \vec{\kappa}+\sum_{\substack{[[a, b]] \leq[m+2,3]] \\ c \leq m+2, b \text { odd }}} P_{b}^{a, c}(\vec{\kappa}) .
$$

Notice that the first part of the statement is more precise. In the second expression, we allow for more terms than the ones that are actually there. In some computations we need the precise expression and hence the first. When using interpolation estimates we use the second expression since it is much shorter and the extra terms do not create any problem in the analysis since they have (at most) the same order of the ones we already have.

Proof. See [10, Lemma 2.3] and [4, Lemma 2.3] in the case that there is no tangential component. For $m=0$ the claim follows directly from (3.9). For $m=1$ we find using (3.10) and (3.9) (and writing as usual $\vec{V}$ for the normal component of the velocity)

$$
\nabla_{t} \nabla_{s} \vec{\kappa}+\nabla_{s}^{5} \vec{\kappa}=\nabla_{s} \nabla_{t} \vec{\kappa}+\left(\langle\vec{\kappa}, \vec{V}\rangle-\partial_{s} \varphi\right) \nabla_{s} \vec{\kappa}+\left[\langle\vec{\kappa}, \vec{\kappa}\rangle \nabla_{s} \vec{V}-\left\langle\nabla_{s} \vec{V}, \vec{\kappa}\right\rangle \vec{\kappa}\right]+\nabla_{s}^{5} \vec{\kappa}
$$




$$
=\nabla_{S}^{3} \vec{V}+\left\langle\nabla_{s} \vec{\kappa}, \vec{V}\right\rangle \vec{\kappa}+\varphi \nabla_{S}^{2} \vec{\kappa}+2\langle\vec{\kappa}, \vec{V}\rangle \nabla_{s} \vec{\kappa}+\langle\vec{\kappa}, \vec{\kappa}\rangle \nabla_{S} \vec{V}+\nabla_{S}^{5} \vec{\kappa}
$$

Since

$$
\begin{aligned}
\nabla_{S}^{3} \vec{V} & =-\nabla_{S}^{5} \vec{\kappa}+\lambda \nabla_{S}^{3} \vec{\kappa}+P_{3}^{3,3}(\vec{\kappa}), \\
\left\langle\nabla_{s} \vec{\kappa}, \vec{V}\right\rangle \vec{\kappa} & =P_{3}^{3,2}(\vec{\kappa})+P_{5}^{1,1}(\vec{\kappa})+\lambda P_{3}^{1,1}(\vec{\kappa}) \\
\text { and } \quad|\vec{\kappa}|^{2} \nabla_{s} \vec{V} & =P_{3}^{3,3}(\vec{\kappa})+P_{5}^{1,1}(\vec{\kappa})+\lambda P_{3}^{1,1}(\vec{\kappa}),
\end{aligned}
$$

we find

$$
\nabla_{t} \nabla_{s} \vec{\kappa}+\nabla_{s}^{5} \vec{\kappa}=P_{3}^{3,3}(\vec{\kappa})+\lambda\left(\nabla_{s}^{3} \vec{\kappa}+P_{3}^{1,1}(\vec{\kappa})\right)+P_{5}^{1,1}(\vec{\kappa})+\varphi \nabla_{s}^{2} \vec{\kappa}
$$

using that $P_{3}^{3,3}(\vec{\kappa})$ allows for more terms than $P_{3}^{3,2}(\vec{\kappa})$. The general statement follows with an induction argument.

Notice that no derivatives of the tangential component $\varphi$ appear. In the following lemma we collect further important formulae.

Lemma 3.6. Suppose $f:[0, T) \times \bar{I} \rightarrow \mathbb{R}^{n}$ is a smooth regular solution to

$$
\partial_{t} f=-\nabla_{s}^{2} \vec{\kappa}-\frac{1}{2}|\vec{\kappa}|^{2} \vec{\kappa}+\lambda \vec{\kappa}+\varphi \partial_{s} f=\vec{V}+\varphi \partial_{s} f
$$

in $(0, T) \times I$. Here $\lambda \in \mathbb{R}$ is a given fixed constant. Then, the following formulae hold on $(0, T) \times I$.

1. For $\mu, d \in \mathbb{N}_{0}, v \in \mathbb{N}$, $v$ odd we have

$$
\nabla_{t} P_{v}^{\mu, d}(\vec{\kappa})=\sum_{\substack{[[a, b]] \leq[\mu+4, v]] \\ c \leq \leq+d \\ b \in[v, v+4], o d d}} P_{b}^{a, c}(\vec{\kappa})+\varphi P_{v}^{\mu+1, d+1}(\vec{\kappa}) .
$$

2. For any $A, C \in \mathbb{N}_{0}, B, N, M \in \mathbb{N}, B$ odd,

$$
\nabla_{t} \sum_{\substack{[[a, b]] \leq[[A, B]] \\ c \leq C \\ b \in[N, M], \text { odd }}} P_{b}^{a, c}(\vec{\kappa})=\sum_{\substack{[[a, b]] \leq[[A+4, B]] \\ c \leq C+4 \\ b \in[N, M+4], \text { odd }}} P_{b}^{a, c}(\vec{\kappa})+\varphi \sum_{\substack{[[a, b]] \leq[[A+1, B]] \\ c \leq C+1 \\ b \in[N, M], \text { odd }}} P_{b}^{a, c}(\vec{\kappa}) .
$$

3. For $\mu, d \in \mathbb{N}_{0}, v \in \mathbb{N}, v$ odd we have

$$
\begin{aligned}
\partial_{t} P_{v}^{\mu, d}(\vec{\kappa})= & \sum_{\substack{[[a, b] \leq \leq[\mu+4, v]] \\
b \in[v, v+d], \text { odd }}} P_{b}^{a, c}(\vec{\kappa})+\varphi P_{v}^{\mu+1, d+1}(\vec{\kappa}) \\
& +\left(\partial_{s} f\right)\left(\varphi\left\langle P_{v}^{\mu, d}(\vec{\kappa}), \vec{\kappa}\right\rangle+\sum_{\substack{[[a, b]] \leq \leq[\mu+3, v+1]] \\
c \leq \max \{d, 3\} \\
b \in[v+1, v+3] \text { even }}} P_{b}^{a, c}(\vec{\kappa})\right),
\end{aligned}
$$

whereas for $v$ even we have

$$
\partial_{t} P_{v}^{\mu, d}(\vec{\kappa})=\sum_{\substack{[[a, b]] \leq[[\mu+4, v]] \\ b \leq 4+d, b \in[v, v+4], \text { even }}} P_{b}^{a, c}(\vec{\kappa})+\varphi P_{v}^{\mu+1, d+1}(\vec{\kappa}) .
$$

4. For any $A, C \in \mathbb{N}_{0}, B, N, M \in \mathbb{N}, B$ odd,

$$
\partial_{t} \sum_{\substack{[[a, b]] \leq[[A, B]] \\ c \leq C \\ b \in[N, M], \text { odd }}} P_{b}^{a, c}(\vec{\kappa})=\sum_{\substack{[[a, b]] \leq[[A+4, B]] \\ c \leq C+4,4 \\ b \in[N, M+4], \text { odd }}} P_{b}^{a, c}(\vec{\kappa})+\varphi \sum_{\substack{[[a, b]] \leq[[A+1, B]] \\[\leq C+1 \\ b \in[N, M], \text { odd }}} P_{b}^{a, c}(\vec{\kappa})
$$




$$
+\left(\partial_{S} f\right)\left(\varphi \sum_{\substack{[[a, b]] \leq[[A, B+1]] \\ c \leq C \\ b \in[N+1, M+1], \text { even }}} P_{b}^{a, c}(\vec{\kappa})+\sum_{\substack{[[a, b]] \leq[[A+3, B+1]] \\ c \leq \max \{C, 3\} \\ b \in[N+1, M+3] \text { even }}} P_{b}^{a, c}(\vec{\kappa})\right),
$$

(Note that the term multiplying $\varphi$ in the tangential component vanishes if we know that $\vec{\kappa}=0$. This fact will be used repeatedly in some computations.) whereas for B even we have

$$
\partial_{t} \sum_{\substack{[[a, b]] \leq[[A, B]] \\ c \leq C \\ b \in[N, M], \text { even }}} P_{b}^{a, c}(\vec{\kappa})=\sum_{\substack{[[a, b]] \leq[[A+4, B]] \\ c \leq C+4 \\ b \in[N, M+4] \text {,even }}} P_{b}^{a, c}(\vec{\kappa})+\varphi \sum_{\substack{[[a, b]] \leq[[A+1, B]] \\ b \leq C+1 \\ b \in[N, M] \text {,even }}} P_{b}^{a, c}(\vec{\kappa}) .
$$

Proof. The proof is obtained by a straight forward generalisation of [8, Lemma 3.1]. We report all details for the sake of the reader in Appendix B.1.

Finally we give some estimates that will be used repeatedly for boundary terms.

Lemma 3.7. We have that for any $x \in[0,1]$ there holds

$$
\begin{aligned}
\left|P_{b}^{a, c}(\vec{\kappa})(x)\right|^{2} & \leq C \int_{I}\left(\left|P_{2 b}^{2 a+1, c+1}(\vec{\kappa})\right|+\left|P_{2 b}^{2 a, c}(\vec{\kappa})\right|\right) d s, \quad \text { if } b \text { is odd, } \\
\left|P_{b}^{a, c}(\vec{\kappa})(x)\right| & \leq C \int_{I}\left(\left|P_{b}^{a+1, c+1}(\vec{\kappa})\right|+\left|P_{b}^{a, c}(\vec{\kappa})\right|\right) d s, \quad \text { if } b \text { is even, }
\end{aligned}
$$

where $C=C\left(\frac{1}{\mathcal{L}(f)}\right)$.

The above lemma will be used in conjunction with interpolation estimates shown below: in particular (3.21) will be used when $b=1$.

Proof. Let $x \in[0,1]$. We first start by showing our statement when $b$ is odd. To motivate our proof's strategy, observe that by embedding theory we know that for any normal vector field $\phi: I \rightarrow \mathbb{R}^{n}$ along $f$ we have

$$
\begin{aligned}
\|\phi\|_{L^{\infty}(I)} & \leq C(n)\left\|\partial_{s} \phi\right\|_{L^{1}(I)}+\frac{C(n)}{\mathcal{L}(f)}\|\phi\|_{L^{1}(I)} \\
& \leq C(n)\left\|\nabla_{s} \phi\right\|_{L^{1}(I)}+C(n)\|\langle\phi, \vec{\kappa}\rangle\|_{L^{1}(I)}+\frac{C(n)}{\mathcal{L}(f)}\|\phi\|_{L^{1}(I)} .
\end{aligned}
$$

On the other hand, to apply interpolation inequalities later on, where the number of factors $b$ must be $b \geq 2$, it is better (instead of applying Cauchy-Schwarz to the $L^{1}$-norms) to consider

$$
\begin{aligned}
\|\phi\|_{L^{\infty}(I)}^{2}=\left\||\phi|^{2}\right\|_{L^{\infty}(I)} & \leq C\left\|\partial_{S}|\phi|^{2}\right\|_{L^{1}(I)}+\frac{C}{\mathcal{L}(f)}\left\||\phi|^{2}\right\|_{L^{1}(I)} \\
& \leq C\left(\frac{1}{\mathcal{L}(f)}\right) \int_{I}|\phi|\left(\left|\nabla_{s} \phi\right|+|\phi|\right) d s,
\end{aligned}
$$

using that $\partial_{S}|\phi|^{2}=2\left\langle\phi, \nabla_{s} \phi\right\rangle$. Then this gives (3.21). When $b$ is even we use the inequality $\|\phi\|_{L^{\infty}(I)} \leq$ $C\left\|\partial_{s} \phi\right\|_{L^{1}(I)}+\frac{C}{\mathcal{L}(f)}\|\phi\|_{L^{1}(I)}$, which holds for any scalar map $\phi$. Choosing $\phi=P_{b}^{a, c}(\vec{\kappa})(x)$ we obtain (3.22).

\subsection{Interpolation inequalities}

Interpolation inequalities are crucial in the proof of long-time existence. Consider the scale invariant norms for $k \in \mathbb{N}_{0}$ and $p \in[1, \infty)$

$$
\|\vec{\kappa}\|_{k, p}:=\sum_{i=0}^{k}\left\|\nabla_{s}^{i} \vec{\kappa}\right\|_{p} \quad \text { with } \quad\left\|\nabla_{S}^{i} \vec{\kappa}\right\|_{p}:=\mathcal{L}(f)^{i+1-1 / p}\left(\int_{I}\left|\nabla_{s}^{i} \vec{\kappa}\right|^{p} d s\right)^{1 / p},
$$


(as in [10]) and the usual $L^{p}$ - norm $\left\|\nabla_{S}^{i} \vec{\kappa}\right\|_{L^{p}}^{p}:=\int_{I}\left|\nabla_{S}^{i} \vec{\kappa}\right|^{p} d s$.

Most of the following results (that we briefly state without proof) can be found in several papers (e.g. $[8,10,17])$. We give the precise reference to the paper where a complete proof can be found. These inequalities are satisfied by closed and open curves and allow also for the boundary points of the curve to move in time. One needs only a control from below on the length of the curve.

Lemma 3.8 (Lemma 4.1 [8]). Let $f: I \rightarrow \mathbb{R}^{n}$ be a smooth regular curve. Then for all $k \in \mathbb{N}, p \geq 2$ and $0 \leq i<k$ we have

$$
\left\|\nabla_{s}^{i} \vec{\kappa}\right\|_{p} \leq C\|\vec{\kappa}\|_{2}^{1-\alpha}\|\vec{\kappa}\|_{k, 2}^{\alpha},
$$

with $\alpha=\left(i+\frac{1}{2}-\frac{1}{p}\right) / k$ and $C=C(n, k, p)$.

Corollary 3.9 (Corollary 4.2 [8]). Let $f: I \rightarrow \mathbb{R}^{n}$ be a smooth regular curve. Then for all $k \in \mathbb{N}$ we have

$$
\|\vec{\kappa}\|_{k, 2} \leq C\left(\left\|\nabla_{s}^{k} \vec{\kappa}\right\|_{2}+\|\vec{\kappa}\|_{2}\right),
$$

with $C=C(n, k)$.

Lemma 3.10 (Lemma 3.4 [5]). Let $f: I \rightarrow \mathbb{R}^{n}$ be a smooth regular curve. For any $a, c, \ell \in \mathbb{N}_{0}, b \in \mathbb{N}, b \geq 2$, $c \leq \ell+2$ and $a<2(\ell+2)$ we find

$$
\int_{I}\left|P_{b}^{a, c}(\vec{\kappa})\right| d s \leq C \mathcal{L}(f)^{1-a-b}\|\vec{\kappa}\|_{2}^{b-\gamma}\|\vec{\kappa}\|_{\ell+2,2}^{\gamma},
$$

with $\gamma=\left(a+\frac{1}{2} b-1\right) /(\ell+2)$ and $C=C(n, \ell, a, b)$. Further if $a+\frac{1}{2} b<2 \ell+5$, then for any $\varepsilon>0$

$$
\int_{I}\left|P_{b}^{a, c}(\vec{\kappa})\right| d s \leq \varepsilon \int_{I}\left|\nabla_{s}^{\ell+2} \vec{\kappa}\right|^{2} d s+C \varepsilon^{-\frac{\gamma}{2-\gamma}}\left(\|\vec{\kappa}\|_{L^{2}}^{2}\right)^{\frac{b-\gamma}{2-\gamma}}+C \mathcal{L}(f)^{1-a-\frac{b}{2}}\|\vec{\kappa}\|_{L^{2}}^{b},
$$

with $C=C(n, \ell, a, b)$.

Lemma 3.11 (Lemma 3.5 [5]). Let $f: I \rightarrow \mathbb{R}^{n}$ be a smooth regular curve and $\ell \in \mathbb{N}_{0}$. If $A, B \in \mathbb{N}$ with $B \geq 2$, and $A+\frac{1}{2} B<2 \ell+5$ then we have

$$
\begin{aligned}
& \sum_{\substack{[[a, b]] \leq[[A, B]] \\
c \leq \ell+2,2 \leq b}} \int_{I}\left|P_{b}^{a, c}(\vec{\kappa})\right| d s \\
& \leq C \min \{1, \mathcal{L}(f)\}^{1-2 A-B} \max \left\{1,\|\vec{\kappa}\|_{2}\right\}^{2 A+B} \max \left\{1,\|\vec{\kappa}\|_{\ell+2,2}\right\}^{\bar{\gamma}},
\end{aligned}
$$

and for any $\varepsilon \in(0,1)$

$$
\begin{aligned}
\sum_{\substack{[[a, b]] \leq[[A, B]] \\
c \leq \ell+2,2 \leq b}} \int_{I}\left|P_{b}^{a, c}(\vec{\kappa})\right| d s \leq & \varepsilon \int_{I}\left|\nabla_{S}^{\ell+2} \vec{\kappa}\right|^{2} d s+C \varepsilon^{-\frac{\bar{\gamma}}{2-\bar{\gamma}}} \max \left\{1,\|\vec{\kappa}\|_{L^{2}}^{2}\right\}^{\frac{2 A+B}{2-\bar{\gamma}}} \\
& +C \min \{1, \mathcal{L}(f)\}^{1-A-\frac{B}{2}} \max \left\{1,\|\vec{\kappa}\|_{L^{2}}\right\}^{2 A+B},
\end{aligned}
$$

with $\bar{\gamma}=\left(A+\frac{1}{2} B-1\right) /(\ell+2)$ and $C=C(n, \ell, A, B)$.

\section{Treatment of the boundary terms}

Similar to [4, Lemma 2.4] we see that at the fixed boundary points the derivatives of the curvature of any even order vanish. 
Lemma 4.1. Let $f$ be a smooth solution of $\partial_{t} f=-\nabla_{S}^{2} \vec{\kappa}+\lambda \vec{\kappa}-\frac{1}{2}|\vec{k}|^{2} \vec{\kappa}+\varphi \tau$ on $(0, T) \times I$ with $\lambda=\lambda(t)$ subject to the boundary conditions $f(t, 1)=P$ (for some $P \in \mathbb{R}^{n}$ ) and $\vec{\kappa}(t, 1)=0$ for all $t \geq 0$. Then $\varphi(t, 1)=0$ and $\nabla_{s}^{2 l} \vec{\kappa}(t, 1)=0$ for all $l \in \mathbb{N}_{0}$ and for all times $t \in(0, T)$.

Proof. For $l=0,1$ the claim follows immediately from the boundary conditions and the fact that $f$ is a smooth solution. Notice that because of the boundary conditions $\varphi(t, 1)=0$ for all $t$. The case $l=2$ is a consequence of (3.9): indeed at $x=1$ we have

$$
0=\nabla_{t} \vec{\kappa}=\nabla_{S}^{2} V=-\nabla_{S}^{4} \vec{\kappa}-\frac{1}{2} \nabla_{S}^{2}\left(|\vec{\kappa}|^{2} \vec{\kappa}\right)+\lambda \nabla_{S}^{2} \vec{\kappa}=-\nabla_{S}^{4} \vec{\kappa} .
$$

The general statement follows from an induction argument. Indeed, assume that the claim is true up to $2 n$, $n \in \mathbb{N}$. By the induction assumption it follows that

$$
\nabla_{t} \nabla_{S}^{2 n-2} \vec{\kappa}(t, 1)=0 \text { for all } t \in(0, T) .
$$

Lemma 3.5 with $m=2 n-2$ gives that at $x=1$

$$
\nabla_{s}^{2 n+2} \vec{\kappa}=P_{3}^{2 n, 2 n}(\vec{\kappa})+\lambda\left(\nabla_{s}^{2 n} \vec{\kappa}+P_{3}^{2 n-2,2 n-2}(\vec{\kappa})\right)+P_{5}^{2 n-2,2 n-2}(\vec{\kappa})+\varphi \nabla_{s}^{2 n-1} \vec{\kappa} .
$$

Since $\varphi(t, 1)=0$ for all $t$ and in all the other terms on the right-hand side there is an odd number of factors and an even number of derivatives, we see that $\nabla_{s}^{2 n+2} \vec{\kappa}(t, 1)=0$ for all $t \in(0, T)$.

We consider now the triple junction where the tangential component plays an important role.

Lemma 4.2. Let $\Gamma=\left\{f_{1}, f_{2}, f_{3}\right\}$ be a smooth solution of (1.10) on $(0, T) \times I$ subject to the boundary conditions (1.9), and assume $\lambda_{i}$ is constant for any $i \in\{1,2,3\}$. Then at $x=0$ (i.e. at the junction point) for any $t \in(0, T)$ we have

$$
\begin{aligned}
& \nabla_{s}^{4} \vec{\kappa}_{i}=\lambda_{i} \nabla_{s}^{2} \vec{\kappa}_{i}+\varphi_{i} \nabla_{s} \vec{\kappa}_{i}, \quad i=1,2,3, \\
& \nabla_{s}^{8} \vec{\kappa}_{i}=\sum_{\substack{[[a, b]] \leq \leq[[6,3]] \\
c \leq 6, j \\
b \text { odd }}} P_{b}^{a, c}\left(\vec{\kappa}_{i}\right)+\varphi_{i} \sum_{\substack{[[a, b]] \leq[[5,1]] \\
c \leq 5 \\
b \text { odd }}} P_{b}^{a, c}\left(\vec{\kappa}_{i}\right)-\varphi_{i}^{2} \nabla_{s}^{2} \vec{\kappa}_{i}-\left(\partial_{t} \varphi_{i}\right) \nabla_{s} \vec{\kappa}_{i},
\end{aligned}
$$

more generally we can write for $m \in \mathbb{N}, m \geq 2$

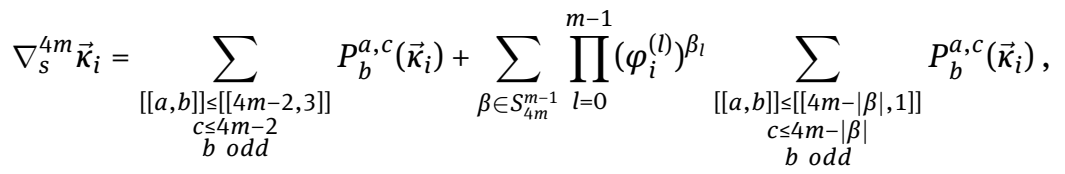

where $\varphi_{i}^{(l)}=\frac{\partial^{l}}{\partial t} \varphi_{i}$ and

$$
S_{i}^{l}:=\left\{\beta=\left(\beta_{0}, \ldots, \beta_{l}\right) \in \mathbb{N}_{0}^{l+1}: 0<|\beta|:=3 \beta_{0}+(3+4) \beta_{1}+\ldots+(3+4 l) \beta_{l}<i\right\} .
$$

Furthermore at $x=0$ (i.e. at the junction point) for any $t \in(0, T)$ we have

$$
\begin{aligned}
& \sum_{i=1}^{3} \nabla_{S}^{5} \vec{\kappa}_{i}=\sum_{i=1}^{3}\left(P_{3}^{3,3}\left(\vec{\kappa}_{i}\right)+2 \lambda_{i} \nabla_{S}^{3} \vec{\kappa}_{i}-\lambda_{i}^{2} \nabla_{s} \vec{\kappa}_{i}+\varphi_{i} \nabla_{S}^{2} \vec{\kappa}_{i}+\left(P_{2}^{4,3}\left(\vec{\kappa}_{i}\right)-\lambda_{i}\left|\nabla_{s} \vec{k}_{i}\right|^{2}\right) \partial_{S} f_{i}\right) \\
& =\sum_{i=1}^{3}\left(\sum_{\substack{[[a, b]] \leq[[3,3]] \\
c \leq 3 \\
b \text { odd }}} P_{b}^{a, c}\left(\vec{\kappa}_{i}\right)+\varphi_{i} \nabla_{s}^{2} \vec{\kappa}_{i}+\left(\partial_{s} f_{i}\right) \sum_{\substack{[[a, b]] \leq[[4,2]] \\
c \leq 3 \\
b \text { even }}} P_{b}^{a, c}\left(\vec{\kappa}_{i}\right)\right) \text {, } \\
& \sum_{i=1}^{3} \nabla_{S}^{9} \vec{\kappa}_{i}=\sum_{i=1}^{3}\left(\sum_{\substack{[[a, b]] \leq[17,3]] \\
c \leq 7 \\
b \text { odd }}} P_{b}^{a, c}\left(\vec{\kappa}_{i}\right)+\varphi_{i} \sum_{\begin{array}{c}
{[[a, b]] \leq[[6,1]]} \\
c \leq 6 \\
b \text { odd }
\end{array}} P_{b}^{a, c}\left(\vec{\kappa}_{i}\right)-\varphi_{i}^{2} \nabla_{S}^{3} \vec{\kappa}_{i}-\partial_{t} \varphi_{i} \nabla_{S}^{2} \vec{\kappa}_{i}\right.
\end{aligned}
$$




$$
\left.+\partial_{s} f_{i}\left[\sum_{\substack{[[a, b]] \leq[[8,2]] \\ c \leq 7 \\ b \text { even }}} P_{b}^{a, c}\left(\vec{\kappa}_{i}\right)+\varphi_{i} \sum_{\substack{[[a, b]] \leq[[5,2]] \\ c \leq 4 \\ b \text { even }}} P_{b}^{a, c}\left(\vec{\kappa}_{i}\right)\right]\right),
$$

more generally we can write for $m \in \mathbb{N}$,

$$
\begin{aligned}
& \sum_{i=1}^{3} \nabla_{s}^{5+4 m} \vec{\kappa}_{i}=\sum_{i=1}^{3}\left(\sum_{\substack{[[a, b]] \leq[[5+4 m-2,3]] \\
c \leq 5+4 m-2 \\
b \text { odd }}} P_{b}^{a, c}\left(\vec{\kappa}_{i}\right)\right. \\
& +\sum_{\beta \in S_{4+4 m}^{m}} \prod_{\substack{l=0 \\
c \leq 5+4 m-|\beta| \\
b \text { odd }}}^{m}\left(\varphi_{i}^{(l)}\right)^{\beta_{l}} \sum_{\substack{[[a, b]] \leq[[5+4 m-|\beta|, 1]] \\
c, c}} P^{\left.a, \vec{\kappa}_{i}\right)}
\end{aligned}
$$

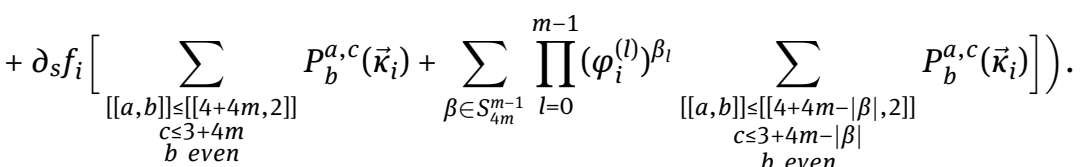

Remark 4.3. In this lemma we see the 'algebra' of the tangential component. More precisely, looking at the sets $S_{i}^{l}$ as defined in (4.4) and, in particular, at the special definition of the length of the multiindex, one sees that a factor $\varphi_{i}$ takes the place of three derivatives of the curvature, while a factor $\partial_{t}^{\ell} \varphi_{i}=\varphi_{i}^{(\ell)}$ takes the place as $3+4 l$ derivatives of the curvature. The order of these terms is given in Lemma 5.3 below.

Proof. Since $\vec{k}(t, 0)=0$ for all $t \in(0, T)$ then (4.1) follows from (3.9) as done in Lemma 4.1 above. Next, since (4.1) holds for any time, we can apply $\nabla_{t}$ to both sides of the equation. Application of Lemma 3.5 and (3.2) give then (4.2). An induction argument using Lemma 3.5 and (3.16) gives then (4.3). Details are given in Appendix B.2.

From the other boundary condition at the triple junction we find that at $x=0$

$$
\partial_{t} \sum_{i=1}^{3}\left(\nabla_{s} \vec{\kappa}_{i}-\lambda_{i} \partial_{s} f_{i}\right)=0 .
$$

Using (3.7), Lemma 3.5, (3.6) and the fact that $\vec{\kappa}_{i}(t, 0)=0$ we get

$$
\begin{aligned}
\sum_{i=1}^{3}( & \left.-\nabla_{s}^{5} \vec{\kappa}_{i}+P_{3}^{3,3}\left(\vec{\kappa}_{i}\right)+\lambda_{i} \nabla_{s}^{3} \vec{\kappa}_{i}+\varphi_{i} \nabla_{s}^{2} \vec{\kappa}_{i}+\left(P_{2}^{4,3}\left(\vec{\kappa}_{i}\right)-\lambda_{i}\left|\nabla_{s} \vec{\kappa}_{i}\right|^{2}-\partial_{t} \lambda_{i}\right) \partial_{s} f_{i}\right) \\
& =\sum_{i=1}^{3} \lambda_{i}\left(-\nabla_{s}^{3} \vec{\kappa}_{i}+\lambda_{i} \nabla_{s} \vec{\kappa}_{i}\right),
\end{aligned}
$$

from which the (4.5) follows. Differentiating in time (4.5), using (3.7), the fact that $\vec{\kappa}=0$, (3.17), (3.18), Lemma 3.5, and (3.6) yield (4.6). In a similar way we obtain by induction (4.7). Details are given in the Appendix B.2.

\section{Treatment of the tangential component}

Here we study the tangential component at the junction point, the only point where the problem gives us information on the $\varphi_{i}$ 's (see Section 2.3). It is exactly here that we need the topological condition that the dimension of the space spanned by the unit tangents at the junction is at least two, see (5.1) below.

Remark 5.1. In order that the curves remain attached, it is necessary that

$$
\partial_{t} f_{i}(t, 0)=\partial_{t} f_{j}(t, 0) \text { for all } t \in(0, T) \text { and } i, j=1,2,3 \text {. }
$$


This follows from differentiating with respect to $t$ the equality $f_{i}(t, 0)=f_{j}(t, 0)$.

The condition is also sufficient. Indeed, since the initial datum is attached we have for any $i, j=1,2,3$

$$
f_{i}(t, 0)=f_{i}(0,0)+\int_{0}^{t} \partial_{t} f_{i}(u, 0) d u=f_{j}(0,0)+\int_{0}^{t} \partial_{t} f_{j}(u, 0) d u=f_{j}(t, 0) .
$$

This gives in particular a condition on the tangential part. Indeed, since the curvature is zero at 0 it is necessary that at 0 for $i, j=1,2,3$

$$
-\nabla_{s}^{2} \vec{\kappa}_{i}+\varphi_{i} \partial_{s} f_{i}=-\nabla_{s}^{2} \vec{\kappa}_{j}+\varphi_{j} \partial_{s} f_{j} .
$$

That is, at zero (i.e. at the triple junction)

$$
\varphi_{i}=-\left\langle\nabla_{s}^{2} \vec{\kappa}_{j}, \partial_{s} f_{i}\right\rangle+\varphi_{j}\left\langle\partial_{s} f_{j}, \partial_{s} f_{i}\right\rangle=\left\langle\partial_{t} f_{j}, \partial_{s} f_{i}\right\rangle .
$$

Let us elaborate on this a bit further. For the sake of notation denote

$$
A_{i}=A_{i}(t):=\left.\nabla_{S}^{2} \vec{\kappa}_{i}\right|_{x=0} \quad \text { and } \quad T_{i}=T_{i}(t)=\partial_{s} f_{i}(t, 0) .
$$

Notice that here (and only in this and the following remark) we denote the tangential vectors at the junction point by $T_{i}$. Also we write $\varphi_{i}$ meaning $\varphi_{i}(t, 0)$. Using the above identity yields that

$$
\begin{aligned}
& \varphi_{i}=-\left\langle A_{i+1}, T_{i}\right\rangle+\varphi_{i+1}\left\langle T_{i+1}, T_{i}\right\rangle, \\
& \varphi_{i}=-\left\langle A_{i+2}, T_{i}\right\rangle+\varphi_{i+2}\left\langle T_{i+2}, T_{i}\right\rangle,
\end{aligned}
$$

and after addition

$$
2 \varphi_{i}-\varphi_{i+1}\left\langle T_{i+1}, T_{i}\right\rangle-\varphi_{i+2}\left\langle T_{i+2}, T_{i}\right\rangle=-\left\langle A_{i+1}+A_{i+2}, T_{i}\right\rangle
$$

for any $i=1,2,3$, where the subindex have to be understood modulo 3 . This yields the system

$$
\left(\begin{array}{ccc}
2 & -\left\langle T_{2}, T_{1}\right\rangle & -\left\langle T_{3}, T_{1}\right\rangle \\
-\left\langle T_{2}, T_{1}\right\rangle & 2 & -\left\langle T_{3}, T_{2}\right\rangle \\
-\left\langle T_{3}, T_{1}\right\rangle & -\left\langle T_{3}, T_{2}\right\rangle & 2
\end{array}\right)\left(\begin{array}{c}
\varphi_{1} \\
\varphi_{2} \\
\varphi_{3}
\end{array}\right)=\left(\begin{array}{c}
-\left\langle A_{2}+A_{3}, T_{1}\right\rangle \\
-\left\langle A_{1}+A_{3}, T_{2}\right\rangle \\
-\left\langle A_{1}+A_{2}, T_{3}\right\rangle
\end{array}\right) .
$$

The above real and symmetric matrix is positive definite (by Sylvester's criterion) if and only if its determinant is strictly positive. A straight forward calculation gives that

$$
\begin{aligned}
\operatorname{det} & =8-2\left(\left\langle T_{3}, T_{2}\right\rangle\right)^{2}-2\left(\left\langle T_{1}, T_{2}\right\rangle\right)^{2}-2\left(\left\langle T_{3}, T_{1}\right\rangle\right)^{2}-2\left\langle T_{1}, T_{2}\right\rangle\left\langle T_{2}, T_{3}\right\rangle\left\langle T_{3}, T_{1}\right\rangle \\
& \geq 2\left(1-\left\langle T_{1}, T_{2}\right\rangle\left\langle T_{2}, T_{3}\right\rangle\left\langle T_{3}, T_{1}\right\rangle\right) \geq 0 .
\end{aligned}
$$

with equality if and only if $T_{1}=T_{2}=T_{3}$ or $\left(T_{i}=T_{i+1}\right.$ and $\left.T_{i+2}=-T_{i}\right)$ for some $i=1,2$, 3 . These degenerate situations are always excluded if we assume that

$$
\operatorname{dim}\left(\operatorname{span}\left\{T_{1}, T_{2}, T_{3}\right\}\right) \geq 2 .
$$

Since the inverse of the matrix is given by

$$
\mathcal{J}=\frac{1}{\operatorname{det}}\left(\begin{array}{ccc}
4-T_{23}^{2} & 2 T_{12}+T_{13} T_{23} & 2 T_{13}+T_{12} T_{23} \\
2 T_{12}+T_{13} T_{23} & 4-T_{13}^{2} & T_{12} T_{13}+2 T_{23} \\
2 T_{13}+T_{12} T_{23} & T_{12} T_{13}+2 T_{23} & 4-T_{12}^{2}
\end{array}\right)
$$

with $T_{i j}=\left\langle T_{i}, T_{j}\right\rangle, i, j=1,2,3$, we see for instance that

$$
\begin{gathered}
\varphi_{1}(0)=-\frac{1}{\operatorname{det}}\left(\left(4-T_{23}^{2}\right)\left\langle A_{2}+A_{3}, T_{1}\right\rangle+\left(2 T_{12}+T_{13} T_{23}\right)\left\langle A_{1}+A_{3}, T_{2}\right\rangle\right. \\
\left.+\left(2 T_{13}+T_{12} T_{23}\right)\left\langle A_{1}+A_{2}, T_{3}\right\rangle\right),
\end{gathered}
$$

and similar formulas hold for $\varphi_{2}(0), \varphi_{3}(0)$. 
Remark 5.2. What we have observed in Remark 5.1 can be repeated for higher order conditions. This allow us to find formula for the derivatives with respect to time of the tangential components $\varphi_{i}$ at the triple junction. More precisely if the flow is sufficiently smooth then we also have

$$
\partial_{t}^{2} f_{i}(t, 0)=\partial_{t}^{2} f_{j}(t, 0) \text { for all } t \in(0, T) \text { and } i, j=1,2,3 \text {. }
$$

Now using (3.7), (3.6) and the fact that $\vec{\kappa}_{i}=0$ at the junction we infer that

$$
\partial_{t}^{2} f_{i}(t, 0)=-\tilde{A}_{i}(t)+\psi_{i}(t) \partial_{s} f^{i}
$$

with normal component (cf. Lemma 3.5 and use $\vec{\kappa}_{i}=0$ )

$$
\begin{aligned}
\tilde{A}_{i} & =\tilde{A}_{i}(t)=\left.\left(\nabla_{t} \nabla_{s}^{2} \vec{\kappa}_{i}-\varphi_{i} \nabla_{s} \vec{V}_{i}\right)\right|_{x=0} \\
& =-\nabla_{s}^{6} \vec{\kappa}_{i}+P_{3}^{4,4}\left(\vec{\kappa}_{i}\right)+\lambda_{i}\left(\nabla_{S}^{4} \vec{\kappa}_{i}+P_{3}^{2,2}\left(\vec{\kappa}_{i}\right)\right)+P_{5}^{2,2}\left(\vec{\kappa}_{i}\right)+\varphi_{i}\left(2 \nabla_{s}^{3} \vec{\kappa}_{i}-\lambda_{i} \nabla_{s} \vec{\kappa}_{i}\right) \\
& =\sum_{\substack{[[a, b]] \leq[[6,1]] \\
c \leq 6 \\
b \text { odd }}} P_{b}^{a, c}\left(\vec{\kappa}_{i}\right)+\varphi_{i}(t, 0) \sum_{\substack{[[a, b]] \leq[[3,1]] \\
c \leq 3 \\
b \text { odd }}} P_{b}^{a, c}\left(\vec{\kappa}_{i}\right),
\end{aligned}
$$

and

$$
\psi_{i}=\partial_{t} \varphi_{i}(t, 0)+\left.\left\langle\nabla_{s} \vec{V}_{i}, \nabla_{S}^{2} \vec{\kappa}_{i}\right\rangle\right|_{x=0} .
$$

For the sake of notation we write again

$$
T_{i}=T_{i}(t)=\partial_{s} f_{i}(t, 0)
$$

Using (5.4) and (5.5) yields that

$$
\begin{aligned}
& \psi_{i}=-\left\langle\tilde{A}_{i+1}, T_{i}\right\rangle+\psi_{i+1}\left\langle T_{i+1}, T_{i}\right\rangle, \\
& \psi_{i}=-\left\langle\tilde{A}_{i+2}, T_{i}\right\rangle+\psi_{i+2}\left\langle T_{i+2}, T_{i}\right\rangle,
\end{aligned}
$$

and after addition

$$
2 \psi_{i}-\psi_{i+1}\left\langle T_{i+1}, T_{i}\right\rangle-\psi_{i+2}\left\langle T_{i+2}, T_{i}\right\rangle=-\left\langle\tilde{A}_{i+1}+\tilde{A}_{i+2}, T_{i}\right\rangle
$$

for any $i=1,2,3$, where the subindex have to be understood modulo 3. This yields the system

$$
\left(\begin{array}{ccc}
2 & -\left\langle T_{2}, T_{1}\right\rangle & -\left\langle T_{3}, T_{1}\right\rangle \\
-\left\langle T_{2}, T_{1}\right\rangle & 2 & -\left\langle T_{3}, T_{2}\right\rangle \\
-\left\langle T_{3}, T_{1}\right\rangle & -\left\langle T_{3}, T_{2}\right\rangle & 2
\end{array}\right)\left(\begin{array}{l}
\psi_{1} \\
\psi_{2} \\
\psi_{3}
\end{array}\right)=\left(\begin{array}{c}
-\left\langle\tilde{A}_{2}+\tilde{A}_{3}, T_{1}\right\rangle \\
-\left\langle\tilde{A}_{1}+\tilde{A}_{3}, T_{2}\right\rangle \\
-\left\langle\tilde{A}_{1}+\tilde{A}_{2}, T_{3}\right\rangle
\end{array}\right) .
$$

which we have already solved in Remark 5.1. Therefore we find again that the matrix is invertible if we assume (5.1). By the expression for the inverse of the matrix given in (5.2), we see for instance from (5.6) that

$$
\begin{aligned}
\partial_{t} \varphi_{1}(0)=-\left.\left\langle\nabla_{s} \vec{V}_{i}, \nabla_{s}^{2} \vec{\kappa}_{i}\right\rangle\right|_{x=0} \\
-\frac{1}{\operatorname{det}}\left(\left(4-T_{23}^{2}\right)\left\langle\tilde{A}_{2}+\tilde{A}_{3}, T_{1}\right\rangle+\left(2 T_{12}+T_{13} T_{23}\right)\left\langle\tilde{A}_{1}+\tilde{A}_{3}, T_{2}\right\rangle\right. \\
\left.+\left(2 T_{13}+T_{12} T_{23}\right)\left\langle\tilde{A}_{1}+\tilde{A}_{2}, T_{3}\right\rangle\right),
\end{aligned}
$$

and similar formulas hold for $\partial_{t} \varphi_{2}(0), \partial_{t} \varphi_{3}(0)$.

More generally an induction argument, that uses (3.6), (3.7), $\vec{\kappa}_{i}=0$ at the boundary, Lemma 3.5, (3.19), and (3.20), gives that for any $m \in \mathbb{N}, m \geq 2$, at the boundary points we have

$$
\partial_{t}^{m} f_{i}=-\tilde{A}_{i}(t)+\psi_{i}(t) \partial_{s} f^{i}
$$

where

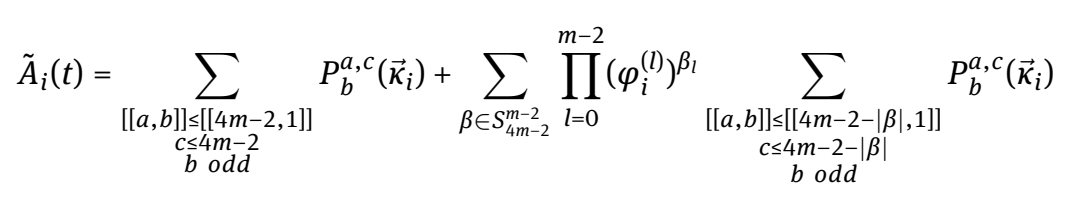


and

$$
\begin{aligned}
\psi_{i}(t)= & \partial_{t}^{m-1} \varphi_{i}(t, 0) \\
& +\sum_{\substack{[[a, b]] \leq[[4 m-3,2]] \\
c \leq 4 m-3 \\
b \text { even }}} P_{b}^{a, c}\left(\vec{\kappa}_{i}\right)+\sum_{\beta \in S_{4(m-1)-2}^{m-3}} \prod_{l=0}^{m-3}\left(\varphi_{i}^{(l)}\right)^{\beta_{l}} \sum_{\begin{array}{c}
{[[a, b]] \leq[[4 m-3-|\beta|, 2]]} \\
c \leq 4 m-3-|\beta| \\
b \text { even }
\end{array}} P_{b}^{a, c}\left(\vec{\kappa}_{i}\right) .
\end{aligned}
$$

Using

$$
\partial_{t}^{m} f_{i}(t, 0)=\partial_{t}^{m} f_{j}(t, 0) \text { for all } t \in(0, T) \text { and } i, j=1,2,3,
$$

and (5.8) the same arguments as above give then for $m \in \mathbb{N}, m \geq 2$

$$
\begin{aligned}
& \partial_{t}^{m-1} \varphi_{1}(0)=\left.\left(\sum_{\substack{[[a, b]] \leq[[4 m-3,2]] \\
c \leq 4 m-3 \\
b \text { even }}} P_{b}^{a, c}\left(\vec{\kappa}_{i}\right)+\sum_{\beta \in S_{4 m-6}^{m-3}} \prod_{l=0}^{m-3}\left(\varphi_{i}^{(l)}\right)^{\beta_{l}} \sum_{\begin{array}{c}
{[[a, b]] \leq[[4 m-3-|\beta|, 2]]} \\
c \leq 4 m-3-|\beta| \\
b \text { even }
\end{array}} P_{b}^{a, c}\left(\vec{\kappa}_{i}\right)\right)\right|_{x=0} \\
&-\frac{1}{\operatorname{det}}\left(\left(4-T_{23}^{2}\right)\left\langle\tilde{A}_{2}+\tilde{A}_{3}, T_{1}\right\rangle+\left(2 T_{12}+T_{13} T_{23}\right)\left\langle\tilde{A}_{1}+\tilde{A}_{3}, T_{2}\right\rangle\right. \\
&\left.+\left(2 T_{13}+T_{12} T_{23}\right)\left\langle\tilde{A}_{1}+\tilde{A}_{2}, T_{3}\right\rangle\right),
\end{aligned}
$$

and similar formulas hold for $\partial_{t}^{m-1} \varphi_{2}(0), \partial_{t}^{m-1} \varphi_{3}(0)$.

Next we give estimates for the tangential components $\varphi_{i}$ (and their time derivatives) at the triple junction. To that end we will use repeatedly Lemma 3.7.

Lemma 5.3. Let $\Gamma=\left\{f_{1}, f_{2}, f_{3}\right\}$ be a smooth solution of (1.10) on $(0, T) \times I$ subject to the boundary conditions (1.9). Furthermore let Assumption 6.1 (see below) hold and assume that there exists a constant $C>0$ such that

$$
\mathcal{L}\left(f_{i}(t)\right) \geq C \text { and }\left\|\vec{\kappa}_{i}(t)\right\|_{2} \leq C \text { for } i=1,2,3,
$$

and any $t \in(0, T)$. Then we have for any $\ell \in \mathbb{N}$ and $i=1,2,3$ that

$$
\begin{gathered}
\left|\varphi_{i}(0)\right| \leq \frac{C}{\delta} \sum_{j=1}^{3} \mathcal{L}\left(f_{j}\right)^{-3}\left\|\vec{\kappa}_{j}\right\|_{2}^{\frac{2 \ell-1}{(\ell+2)}}\left\|\vec{\kappa}_{j}\right\|_{\ell+2,2}^{\frac{5}{2(\ell+2)}} \leq \frac{C}{\delta} \sum_{j=1}^{3}\left\|\vec{\kappa}_{j}\right\|_{\ell+2,2}^{\frac{5}{2(\ell+2)}}, \\
\left|\partial_{t} \varphi_{i}(0)\right| \leq \frac{C}{\delta} \sum_{j=1}^{3} \max \left\{1,\left\|\vec{\kappa}_{j}\right\|_{\ell+2,2}\right\}^{\frac{13}{2(\ell+2)}} \quad \text { for } \ell \geq 5 .
\end{gathered}
$$

More generally, we have for any $\ell \in \mathbb{N}$ and $i=1,2,3$ that

$$
\left|\partial_{t}^{m} \varphi_{i}(0)\right| \leq C\left(m, \frac{1}{\delta}\right) \sum_{j=1}^{3} \max \left\{1,\left\|\vec{\kappa}_{j}\right\|_{\ell+2,2}\right\}^{\frac{5+8 m}{2(\ell+2)}} \quad \text { for } \ell \geq 4 m+1 .
$$

Proof. An expression for $\varphi_{i}(t, 0)$ is given in Remark 5.1 (see (5.3) for $\left.\varphi_{1}\right)$. Again we write here $\varphi_{i}(0)$ meaning $\varphi_{i}(t, 0)$ for $t \in(0, T)$. Using Assumption 6.1 we find with Lemma 4.1 and Lemma 3.10 (for any $\ell \geq 1$ )

$$
\begin{aligned}
\left|\varphi_{i}(0)\right| & \leq \frac{C}{\delta} \sum_{j=1}^{3}\left|\nabla_{s}^{2} \vec{\kappa}_{j}(0)\right|=\frac{C}{\delta} \sum_{j=1}^{3}\left(\left|\nabla_{s}^{2} \vec{\kappa}_{j}(0)\right|^{2}-\left|\nabla_{s}^{2} \vec{\kappa}_{j}(1)\right|^{2}\right)^{\frac{1}{2}} \\
& \leq \frac{C}{\delta} \sum_{j=1}^{3}\left(-2 \int_{0}^{1}\left\langle\nabla_{s}^{3} \vec{k}_{j}, \nabla_{s}^{2} \vec{k}_{j}\right\rangle d s\right)^{\frac{1}{2}} \leq \frac{C}{\delta} \sum_{j=1}^{3} \mathcal{L}\left(f_{j}\right)^{-3}\left\|\vec{k}_{j}\right\|_{2}^{\frac{2 \ell(\ell+2)}{2(\ell+2)}}\left\|\vec{k}_{j}\right\|_{\ell+2,2}^{\frac{5}{2(\ell+2)}} .
\end{aligned}
$$

An expression for $\partial_{t} \varphi_{i}(t, 0)$ is given in Remark 5.2 (see for instance (5.7) for $\left.\partial_{t} \varphi_{1}\right)$. Again using Assumption 6.1 we find

$$
\left|\partial_{t} \varphi_{i}(0)\right| \leq\left|\left\langle\nabla_{s} \vec{V}_{i}, \nabla_{s}^{2} \vec{\kappa}_{i}\right\rangle\right|_{x=0} \mid
$$




$$
+\frac{C}{\delta} \sum_{j=1}^{3}\left|\sum_{\substack{[[a, b]] \leq[6,1]] \\ c \leq 6 \\ b \text { odd }}} P_{b}^{a, c}\left(\vec{\kappa}_{j}\right)\right|_{x=0}+\left.\varphi_{j}(t, 0) \sum_{\substack{[[a, b]] \leq[[3,1]] \\ c \leq 3 \\ b \text { odd }}} P_{b}^{a, c}\left(\vec{\kappa}_{j}\right)\right|_{x=0} \mid
$$

By (3.21), Lemma 3.11 and the uniform bound on length and the $L^{2}$-norm of the curvature we infer for any $\ell \geq 5$

$$
\sum_{\substack{[[a, b]] \leq[[6,1]] \\ c \leq 6 \\ b \text { odd }}}\left|P_{b}^{a, c}\left(\vec{\kappa}_{j}\right)\right|(0) \leq C\left(\sum_{\substack{[[a, b]] \leq[13,2]] \\ c \leq 7 \\ b \geq 2}} \int_{I}\left|P_{b}^{a, c}\left(\vec{\kappa}_{j}\right)\right| d s\right)^{\frac{1}{2}} \leq C \max \left\{1,\left\|\vec{\kappa}_{j}\right\|_{\ell+2,2}\right\}^{\frac{13}{2(\ell+2)}},
$$

and

$$
\sum_{\substack{[[a, b]] \leq[[3,1]] \\ c \leq 3 \\ b \text { odd }}}\left|P_{b}^{a, c}\left(\vec{\kappa}_{j}\right)\right|(0) \leq C\left(\sum_{\substack{[[a, b]] \leq[[7,2]] \\ c \leq 4 \\ b \geq 2}} \int_{I}\left|P_{b}^{a, c}\left(\vec{\kappa}_{j}\right)\right| d s\right)^{\frac{1}{2}} \leq C \max \left\{1,\left\|\vec{\kappa}_{j}\right\|_{\ell+2,2}\right\}^{\frac{7}{2(\ell+2)}}
$$

Also, using once again that $\nabla_{S}^{2} \vec{\kappa}_{i}(1)=0$, by Lemma 3.11

$$
\begin{aligned}
\left|\left\langle\nabla_{s} \vec{V}_{i}, \nabla_{s}^{2} \vec{\kappa}_{i}\right\rangle\right|_{x=0} & =\sum_{\substack{[[a, b]] \leq[[5,2]] \\
c \leq 3, b \geq 2, b \text { even }}}\left|P_{b}^{a, c}\left(\vec{\kappa}_{i}\right)(0)-P_{b}^{a, c}\left(\vec{\kappa}_{i}\right)(1)\right| \\
& \leq \sum_{\substack{[[a, b]] \leq[6,2]] \\
c \leq 4, b \geq 2}} \int_{I}\left|P_{b}^{a, c}\left(\vec{\kappa}_{i}\right)\right| d s \leq C \max \left\{1,\left\|\vec{\kappa}_{i}\right\|_{\ell+2,2}\right\}^{\frac{6}{\ell+2}} .
\end{aligned}
$$

Therefore we get

$$
\begin{aligned}
\left|\partial_{t} \varphi_{i}(0)\right| & \leq C \max \left\{1,\left\|\vec{\kappa}_{i}\right\|_{\ell+2,2}\right\}^{\frac{6}{\ell+2}}+\frac{C}{\delta} \sum_{j=1}^{3} \max \left\{1,\left\|\vec{\kappa}_{j}\right\|_{\ell+2,2}\right\}^{\frac{13}{2(\ell+2)}} \\
& +\frac{C}{\delta} \sum_{j=1}^{3} \max \left\{1,\left\|\vec{\kappa}_{j}\right\|_{\ell+2,2}\right\}^{\frac{7}{2(\ell+2)}}\left|\varphi_{j}(0)\right|
\end{aligned}
$$

Using (5.13) we finally infer

$$
\begin{aligned}
\left|\partial_{t} \varphi_{i}(0)\right| \leq C & \max \left\{1,\left\|\vec{\kappa}_{i}\right\|_{\ell+2,2}\right\}^{\frac{12}{2(\ell+2)}}+\frac{C}{\delta} \sum_{j=1}^{3} \max \left\{1,\left\|\vec{\kappa}_{j}\right\|_{\ell+2,2}\right\}^{\frac{13}{2(\ell+2)}} \\
& +\frac{C}{\delta} \sum_{j=1}^{3} \max \left\{1,\left\|\vec{\kappa}_{j}\right\|_{\ell+2,2}\right\}^{\frac{7}{2(\ell+2)}} \sum_{r=1}^{3}\left\|\vec{\kappa}_{r}\right\|_{\ell+2,2}^{\frac{5}{2(\ell+2)}} \\
\leq & \frac{C}{\delta} \sum_{j=1}^{3} \max \left\{1,\left\|\vec{\kappa}_{j}\right\|_{\ell+2,2}\right\}^{\frac{13}{2(\ell+2)}}
\end{aligned}
$$

where we have used $\left\|\overrightarrow{\boldsymbol{k}}_{r}\right\|_{\ell+2,2}^{\frac{5}{2(\ell+2)}} \leq \max \left\{1,\left\|\overrightarrow{\boldsymbol{k}}_{r}\right\|_{\ell+2,2}\right\}^{\frac{5}{2(\ell+2)}} \leq \max \left\{1,\left\|\vec{\kappa}_{r}\right\|_{\ell+2,2}\right\}^{\frac{6}{2(\ell+2)}}$ and Young inequality $|a||b| \leq$ $C\left(|a|^{p}+|b|^{q}\right)$ with $p=13 / 7$ and $q=13 / 6$ for the product of terms of type $a=\left\{1,\left\|\vec{\kappa}_{j}\right\|_{\ell+2,2}\right\}^{\frac{7}{2(\ell+2)}}$ and $b=$ $\max \left\{1,\left\|\vec{\kappa}_{r}\right\|_{\ell+2,2}\right\}^{\frac{6}{2(\ell+2)}}$. The general statement (5.15) follows by an induction argument, that uses (5.11), (5.9), Lemma 3.7, Lemma 3.11, and Lemma A.2. Let us look at the single terms. First we estimate the terms appearing in (5.9) (where $m$ is replaced by $m+1$ ). Using (3.21), (5.12), and Lemma 3.11 we have for $\ell \geq 4 m+1$

$$
\begin{aligned}
\sum_{\substack{[[a, b]] \leq[[4 m+2,1]] \\
c \leq 4 m+2 \\
b \text { odd }}}\left|P_{b}^{a, c}\left(\vec{\kappa}_{j}\right)\right|(0) & \left.\leq C \sum_{\substack{[[a, b]] \leq[[8 m+5,2]] \\
c \leq 4 m+3 \\
b \text { even }}} \int_{I}\left|P_{b}^{a, c}\left(\vec{\kappa}_{j}\right)\right| d s\right)^{1 / 2} \\
& \leq C \max \left\{1,\left\|\vec{\kappa}_{j}\right\|_{\ell+2,2}\right\}^{\frac{5+8 m}{2(\ell+2)}}
\end{aligned}
$$


and similarly together with the induction assumptions

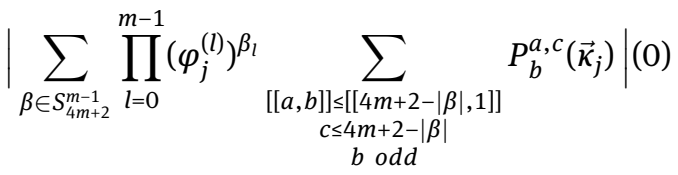

$$
\begin{aligned}
& \leq C \sum_{\beta \in S_{4 m+2}^{m-1}} \prod_{l=0}^{m-1}\left|\varphi_{j}^{(l)}\right|^{\beta_{l}}\left(\sum_{\begin{array}{c}
{[[a, b]] \leq[[8 m+5-2|\beta|, 2]]} \\
c \leq 4 m+3-|\beta| \\
b \text { even }
\end{array}} \int_{I}\left|P_{b}^{a, c}\left(\vec{\kappa}_{j}\right)\right| d s\right)^{1 / 2} \\
& \leq C \sum_{\beta \in S_{4 m+2}^{m-1}} \prod_{l=0}^{m-1}\left|\varphi_{j}^{(l)}\right|^{\beta_{l}} \max \left\{1,\left\|\vec{\kappa}_{j}\right\|_{\ell+2,2}\right\}^{\frac{5+8 m-2|\beta|}{2(\ell+2)}} \\
& \leq C \sum_{\beta \in S_{4 m+2}^{m-1}} \prod_{l=0}^{m-1}\left(C\left(l, \frac{1}{\delta}\right) \sum_{k=1}^{3} \max \left\{1,\left\|\vec{\kappa}_{k}\right\|_{\ell+2,2}\right\}^{\frac{5+8 l}{2(\ell+2)}}\right)^{\beta_{l}} \max \left\{1,\left\|\vec{\kappa}_{j}\right\|_{\ell+2,2}\right\}^{\frac{5+8 m-2|\beta|}{2(\ell+2)}} \\
& \leq C\left(m, \frac{1}{\delta}\right) \sum_{k=1}^{3} \max \left\{1,\left\|\vec{\kappa}_{k}\right\|_{\ell+2,2}\right\}^{\frac{5+8 m}{2(\ell+2)}}
\end{aligned}
$$

where we have used Lemma A.2 with $\gamma=\frac{5+8 m}{2(\ell+2)}$ (on recalling (4.4) note that $\sum_{l=0}^{m-1} \beta_{l}(5+8 \ell)<\sum_{l=0}^{m-1} 2 \beta_{l}(3+$ $4 \ell)=2|\beta|$.) For the remaining terms in (5.11) (with $m$ replaced by $m+1$ ) we observe that

$$
\begin{aligned}
& \left|\sum_{\substack{[[a, b]] \leq[[4 m+1,2]] \\
c \leq 4 m+1 \\
b \text { even }}} P_{b}^{a, c}\left(\vec{\kappa}_{i}\right)+\sum_{\beta \in S_{4 m-2}^{m-2}} \prod_{l=0}^{m-2}\left(\varphi_{i}^{(l)}\right)^{\beta_{l}} \sum_{\begin{array}{c}
{[[a, b]] \leq[[4 m+1-|\beta|, 2]]} \\
c \leq 4 m+1-|\beta| \\
b \text { even }
\end{array}} P_{b}^{a, c}\left(\vec{\kappa}_{i}\right)\right|(0) \\
& \leq C \max \left\{1,\left\|\vec{\kappa}_{i}\right\|_{\ell+2,2}\right\}^{\frac{8 m+4}{2(\ell+2)}} \\
& +C \sum_{\beta \in S_{4 m-2}^{m-2}} \prod_{l=0}^{m-2}\left(C\left(l, \frac{1}{\delta}\right) \sum_{k=1}^{3} \max \left\{1,\left\|\vec{\kappa}_{k}\right\|_{\ell+2,2}\right\}^{\left.\frac{5+8 l}{2(\ell+2)}\right)^{\beta_{l}}} \max \left\{1,\left\|\vec{\kappa}_{i}\right\|_{\ell+2,2}\right\}^{\frac{8 m+4-2|\beta|}{2(\ell+2)}}\right. \\
& \leq C\left(m, \frac{1}{\delta}\right) \sum_{k=1}^{3} \max \left\{1,\left\|\vec{\kappa}_{k}\right\|_{\ell+2,2}\right\}^{\frac{5+8 m}{2(\ell+2)}}
\end{aligned}
$$

where we have used Lemma 3.7, (5.12), Lemma 3.11, and Lemma A.2. The claim now follows from (5.11) putting all estimates together.

\section{Long-time existence result}

Assumption 6.1. We assume that on the maximal time interval of existence $[0, T)$

1. the length of each of the curves remains uniformly bounded from below away from zero;

2. there exists $a 1 \geq \delta>0$ such that for any $t \in[0, T)$ there exist $i=i(t), j=j(t) \in\{1,2,3\}$ with $\left|\left\langle\partial_{s} f_{i}(t, 0), \partial_{s} f_{j}(t, 0)\right\rangle\right| \leq 1-\delta$.

Remark 6.2. The first assumption is necessary to be able to use interpolation inequalities. Under the second assumption the determinant of the matrix in Remark 5.1 is bounded from below uniformly by $2 \delta$. Moreover

$$
\operatorname{dim}\left(\operatorname{span}\left\{\partial_{s} f_{1}(t, 0), \partial_{s} f_{2}(t, 0), \partial_{s} f_{3}(t, 0)\right\}\right) \geq 2 \text { for all } t \in[0, T) .
$$

Proof of Theorem 1.1. First of all let us remark that in the following the constant $C$ may change from line to line. A short time existence result gives that a solution $\Gamma=\left\{f_{1}, f_{2}, f_{3}\right\}$ exists in a small time interval, see 
Section 2.3. In particular (see again Section 2.3) the tangential components $\varphi_{i}$ grow linearly in the interior of each curves. We will give notice when this fact plays a role in the proof.

We assume by contradiction that the solution does not exist globally in time, that is there exists $0<T<\infty$ where $T$ denotes the maximal time of existence. In view of our Assumption 6.1 this implies that at least one curve ceases to be smooth or regular at $t=T$.

Since (1.10) is a gradient flow, the energy is decreasing in time and in particular the $L^{2}$-norm of the curvature is uniformly bounded in $(0, T)$. Indeed,

$$
\max _{i=1,2,3}\left\|\vec{\kappa}_{i}\right\|_{L^{2}}^{2} \leq 2 \sum_{i=1}^{3} \varepsilon_{\lambda}\left(f_{i}\right) \leq 2 \sum_{i=1}^{3} \varepsilon_{\lambda}\left(f_{i, 0}\right)<\infty .
$$

Similarly, since $\lambda_{i}>0, i=1,2,3$, the length of the curves remains uniformly bounded from above.

First Step Uniform estimate of $\left\|\nabla_{S}^{2} \vec{\kappa}_{i}\right\|_{L^{2}}$ on $(0, T), i=1,2,3$.

We wish now to estimate the derivatives of the curvature. We use here that the normal velocity in (1.10) can be written as

$$
-\nabla_{s}^{2} \vec{\kappa}_{i}+P_{3}^{0,0}\left(\vec{\kappa}_{i}\right)+\lambda_{i} \vec{\kappa}_{i} \quad i=1,2,3,
$$

and we simplify as much as possible the notation using the $P_{b}^{a, c}\left(\vec{\kappa}_{i}\right)$. Using Lemma 3.2 (taking $\vec{\phi}=\nabla_{s}^{2} \overrightarrow{\kappa_{i}}$ ) and Lemma 3.5, and summing over $i$ we find

$$
\begin{aligned}
& \sum_{i=1}^{3}\left(\frac{d}{d t} \frac{1}{2} \int_{I}\left|\nabla_{s}^{2} \vec{\kappa}_{i}\right|^{2} d s+\int_{I}\left|\nabla_{S}^{4} \vec{\kappa}_{i}\right|^{2} d s\right) \\
& =\sum_{i=1}^{3}\left(-\left[\left\langle\nabla_{s}^{2} \vec{\kappa}_{i}, \nabla_{S}^{5} \vec{\kappa}_{i}\right\rangle\right]_{0}^{1}+\left[\left\langle\nabla_{s}^{3} \vec{\kappa}_{i}, \nabla_{S}^{4} \vec{\kappa}_{i}\right\rangle\right]_{0}^{1}+\int_{I}\left(P_{4}^{6,4}\left(\vec{\kappa}_{i}\right)+P_{4}^{6,2}\left(\vec{\kappa}_{i}\right)+P_{6}^{4,2}\left(\vec{\kappa}_{i}\right)\right) d s\right) \\
& \quad+\sum_{i=1}^{3}\left(\int_{I}\left\langle\varphi_{i} \nabla_{s}^{3} \vec{\kappa}_{i}+\frac{1}{2} \nabla_{S}^{2} \vec{\kappa}_{i} \varphi_{i, s}, \nabla_{S}^{2} \vec{\kappa}_{i}\right\rangle d s+\lambda_{i} \int_{I}\left(\left\langle\nabla_{s}^{2} \vec{\kappa}_{i}, \nabla_{S}^{4} \vec{\kappa}_{i}\right\rangle+P_{4}^{4,2}\left(\vec{\kappa}_{i}\right)\right) d s\right) \\
& =\sum_{i=1}^{3}\left(-\left[\left\langle\nabla_{S}^{2} \vec{\kappa}_{i}, \nabla_{S}^{5} \vec{\kappa}_{i}\right\rangle\right]_{0}^{1}+\left[\left\langle\nabla_{S}^{3} \vec{\kappa}_{i}, \nabla_{S}^{4} \vec{\kappa}_{i}\right\rangle\right]_{0}^{1}+\frac{1}{2}\left[\varphi_{i}\left|\nabla_{s}^{2} \vec{\kappa}_{i}\right|^{2}\right]_{0}^{1}+\sum_{[[a, b]] \leq[[6,4]]} \int_{I} P_{b}^{a, c}\left(\vec{\kappa}_{i}\right) d s\right)
\end{aligned}
$$

where we have used the fact that $\lambda_{i}$ are given constants. Summing on both sides $\frac{1}{2} \int_{I}\left|\nabla_{s}^{2} \vec{\kappa}_{i}\right|^{2} d s$ we find

$$
\begin{aligned}
& \sum_{i=1}^{3}\left(\frac{d}{d t} \frac{1}{2} \int_{I}\left|\nabla_{s}^{2} \vec{\kappa}_{i}\right|^{2} d s+\frac{1}{2} \int_{I}\left|\nabla_{s}^{2} \vec{\kappa}_{i}\right|^{2} d s+\int_{I}\left|\nabla_{s}^{4} \vec{\kappa}_{i}\right|^{2} d s\right) \\
= & \sum_{i=1}^{3}\left(-\left[\left\langle\nabla_{s}^{2} \vec{\kappa}_{i}, \nabla_{s}^{5} \vec{\kappa}_{i}\right\rangle\right]_{0}^{1}+\left[\left\langle\nabla_{s}^{3} \vec{\kappa}_{i}, \nabla_{s}^{4} \vec{\kappa}_{i}\right\rangle\right]_{0}^{1}+\frac{1}{2}\left[\varphi_{i}\left|\nabla_{s}^{2} \vec{\kappa}_{i}\right|^{2}\right]_{0}^{1}+\sum_{\substack{[[a, b]] \leq[[6,4]] \\
c \leq 4,2 \leq b}} \int_{I} P_{b}^{a, c}\left(\vec{\kappa}_{i}\right) d s\right) .
\end{aligned}
$$

Using Lemma 3.11 (with $A=6, B=4, C \leq 4, \ell=2$ ) one sees that the integrals on the right hand side can be controlled as follows

$$
\sum_{i=1}^{3}\left(\sum_{\substack{[a, b]] \leq[[6,4]] \\ c \leq 4,2 \leq b}} \int_{I} P_{b}^{a, c}\left(\vec{\kappa}_{i}\right) d s\right) \leq \varepsilon \sum_{i=1}^{3} \int_{0}^{1}\left|\nabla_{s}^{4} \vec{\kappa}_{i}\right|^{2} d s+C_{\epsilon}\left(\lambda_{i}, f_{i, 0}, \mathcal{L}\left(f_{i}\right), \varepsilon_{\lambda_{i}}\left(f_{i, 0}\right)\right)
$$

with $\varepsilon \in(0,1)$. Here we have used that fact that the length of the curves remains bounded away from zero. It remains to consider the boundary terms.

At the fixed boundary points (i.e. at $x=1$ ) all even derivatives of the curvature are zero by Lemma 4.1 and hence in reality we have a contribution from the boundary terms only from the junction point. As one can 
immediately see we have high derivatives on the boundary terms but using the boundary condition as done in Lemma 4.2 we can lower the order of the boundary terms. Let consider the three terms separately. Since $\vec{\kappa}=0$ at the boundary and at the junction point $\partial_{t} f_{i}=\partial_{t} f_{j}$ we can write

$$
\left.\sum_{i=1}^{3}\left\langle-\nabla_{S}^{2} \vec{\kappa}_{i}, \nabla_{S}^{5} \vec{\kappa}_{i}\right\rangle\right|_{x=0}=\left.\sum_{i=1}^{3}\left\langle\partial_{t} f_{i}, \nabla_{S}^{5} \vec{\kappa}_{i}\right\rangle\right|_{x=0}=\left.\left\langle\partial_{t} f_{1}, \sum_{i=1}^{3} \nabla_{S}^{5} \vec{\kappa}_{i}\right\rangle\right|_{x=0} .
$$

Then by Lemma 4.2 at $x=0$ (recall that $\vec{\kappa}=0$ at $x=0$, therefore $\partial f_{i}=-\nabla_{s}^{2} \vec{\kappa}_{i}+\varphi_{i} \partial_{s} f_{i}$ )

$$
\begin{aligned}
& \sum_{i=1}^{3}\left\langle-\nabla_{s}^{2} \vec{\kappa}_{i}, \nabla_{s}^{5} \vec{\kappa}_{i}\right\rangle \\
& =\left\langle\partial_{t} f_{1}, \sum_{i=1}^{3}\left(P_{3}^{3,3}\left(\vec{\kappa}_{i}\right)+2 \lambda_{i} \nabla_{s}^{3} \vec{\kappa}_{i}-\lambda_{i}^{2} \nabla_{s} \vec{\kappa}_{i}+\varphi_{i} \nabla_{s}^{2} \vec{\kappa}_{i}+\left(P_{2}^{4,3}\left(\vec{\kappa}_{i}\right)-\lambda_{i}\left|\nabla_{s} \vec{\kappa}_{i}\right|^{2}\right) \partial_{s} f_{i}\right)\right\rangle \\
& =\sum_{i=1}^{3}\left\langle\partial_{t} f_{i}, P_{3}^{3,3}\left(\vec{\kappa}_{i}\right)+2 \lambda_{i} \nabla_{s}^{3} \vec{\kappa}_{i}-\lambda_{i}^{2} \nabla_{s} \vec{\kappa}_{i}+\varphi_{i} \nabla_{s}^{2} \vec{\kappa}_{i}+\left(P_{2}^{4,3}\left(\vec{\kappa}_{i}\right)-\lambda_{i}\left|\nabla_{s} \vec{\kappa}_{i}\right|^{2}\right) \partial_{s} f_{i}\right\rangle \\
& =\sum_{i=1}^{3}\left(\sum_{\substack{[a, b]] \leq \leq[5,4]] \\
c \leq 3,2 \leq b \\
b \text { even }}} P_{b}^{a, c}\left(\vec{\kappa}_{i}\right)+\varphi_{i}\left(-\left|\nabla_{s}^{2} \vec{k}_{i}\right|^{2}+P_{2}^{4,3}\left(\vec{\kappa}_{i}\right)-\lambda_{i}\left|\nabla_{s} \vec{\kappa}_{i}\right|^{2}\right)\right),
\end{aligned}
$$

where we have used the fact that $\lambda_{i}$ are constant. By (3.22) we get

$$
\left.\sum_{\substack{[[a, b]] \leq[[5,4]] \\
c \leq 3,2 \leq b \\
b \text { even }}} P_{b}^{a, c}\left(\vec{\kappa}_{i}\right)\right|_{x=0} \leq C \int_{\substack{I\left[\begin{array}{c}
[a, b]] \leq[[6,4]] \\
c \leq 4,2 \leq b \\
b \text { even }
\end{array} \\
\mid\right.}}\left|P_{b}^{a, c}\left(\vec{\kappa}_{i}\right)\right| d s .
$$

Thanks to the interpolation inequality (3.27) (together with the uniform control on the lengths from below, see Assumption 6.1) we find that the above terms can be controlled via absorbtion into the terms $\left\|\nabla_{s}^{4} \vec{\kappa}_{i}\right\|_{L^{2}}^{2}$. Next we study the term that depends on the tangential component of the flow equation and hence is new compared to our previous studies ([4], [5], [8], [17], [9]). The term we need to control is

$$
\left.\varphi_{i}\left(-\left|\nabla_{s}^{2} \vec{\kappa}_{i}\right|^{2}+P_{2}^{4,3}\left(\vec{\kappa}_{i}\right)-\lambda_{i}\left|\nabla_{s} \vec{\kappa}_{i}\right|^{2}\right)\right|_{x=0}=\left.\varphi_{i} \sum_{\substack{[a, b]] \leq[[4,2]] \\ c \leq 3,2 \leq b \\ b \text { even }}} P_{b}^{a, c}\left(\vec{\kappa}_{i}\right)\right|_{x=0} .
$$

The factor $\varphi_{i}(0)$ can be expressed using the formulas for the tangential component of the velocity at $x=0$ (see (5.3) for $\varphi_{1}$ ). Then by Assumption 6.1 and (5.13) (with $\ell=2$ ) we find

$$
\left|\varphi_{i}(0)\right| \leq \frac{C}{\delta} \sum_{j=1}^{3} \mathcal{L}\left(f_{j}\right)^{-3}\left\|\vec{k}_{j}\right\|_{2}^{\frac{3}{8}}\left\|\vec{\kappa}_{j}\right\|_{4,2}^{\frac{5}{8}} .
$$

For the other factor in (6.4) we use (3.22), Lemma 3.11 and the uniform bounds on the lengths to write

$$
\left|\sum_{\substack{[[a, b]] \leq[[4,2]] \\ c \leq 3,2 \leq b \\ b \text { even }}} P_{b}^{a, c}\left(\vec{\kappa}_{i}\right)\right|_{x=0}\left|\leq C \sum_{\substack{[[a, b]] \leq[[5,2]] \\ c \leq 4,2 \leq b}} \int_{I}\right| P_{b}^{a, c}\left(\vec{\kappa}_{i}\right) \mid d s \leq C \max \left\{1,\left\|\vec{\kappa}_{i}\right\|_{4,2}^{\frac{5}{4}}\right\}
$$

Combining the estimates above and using the uniform bounds on the lengths we infer

$$
\begin{aligned}
& \left|\sum_{i=1}^{3} \varphi_{i}\left(-\left|\nabla_{S}^{2} \vec{\kappa}_{i}\right|^{2}+P_{2}^{4,3}\left(\vec{\kappa}_{i}\right)+\lambda_{i}\left|\nabla_{s} \vec{\kappa}_{i}\right|^{2}\right)\right|_{x=0} \mid \\
& \leq \sum_{i=1}^{3}\left(\sum_{j=1}^{3} C\left(\mathcal{L}\left(f_{j}\right), \delta\right) \max \left\{1,\left\|\vec{\kappa}_{j}\right\|_{4,2}\right\}^{\frac{5}{8}}\right) \max \left\{1,\left\|\vec{\kappa}_{i}\right\|_{4,2}\right\}^{\frac{5}{4}} \leq \sum_{i=1}^{3} C \max \left\{1,\left\|\vec{\kappa}_{i}\right\|_{4,2}\right\}^{\frac{15}{8}}
\end{aligned}
$$


using Young's inequality $|a b| \leq C\left(|a|^{p}+|b|^{q}\right)$ with $p=3$ and $q=\frac{3}{2}$ on product terms of type $b=$ $\max \left\{1,\left\|\vec{\kappa}_{i}\right\|_{4,2}\right\}^{\frac{5}{4}}, a=\max \left\{1,\left\|\vec{\kappa}_{j}\right\|_{4,2}\right\}^{\frac{5}{8}}$. Since the exponent of the factor $\left\|\vec{\kappa}_{i}\right\|_{4,2}$ is smaller than 2 we can control this term.

Proceeding similarly for the second boundary term in (6.1) we find with Lemma 4.2 for $i=1,2,3$

$$
\left.\left\langle\nabla_{s}^{3} \vec{\kappa}_{i}, \nabla_{S}^{4} \vec{\kappa}_{i}\right\rangle\right|_{0} ^{1}=\left.\lambda_{i}\left\langle\nabla_{S}^{3} \vec{\kappa}_{i}, \nabla_{s}^{2} \vec{\kappa}_{i}\right\rangle\right|_{0} ^{1}+\left.\varphi_{i}\left\langle\nabla_{s}^{3} \vec{\kappa}_{i}, \nabla_{s} \vec{\kappa}_{i}\right\rangle\right|_{0} ^{1} .
$$

As before with (3.22) and Lemma 3.11 the first term on the right hand side can be controlled by $\left\|\nabla_{s}^{4} \vec{\kappa}\right\|_{2}$ since $\lambda_{i}$ are constant and by Assumption 6.1. The second term coincides with one of the term coming from the tangential component treated above and hence it is also controlled.

The last boundary term in (6.1) coincides with terms that we have already treated and hence this also behaves as $\left\|\vec{\kappa}_{i}\right\|_{4,2}^{\frac{15}{8}}$. By Corollary 3.9 and Young's inequality we finally achieve that for $\varepsilon \in(0,1)$

$$
\begin{gathered}
\sum_{i=1}^{3}\left(-\left[\left\langle\nabla_{s}^{2} \vec{\kappa}_{i}, \nabla_{s}^{5} \vec{\kappa}_{i}\right\rangle\right]_{0}^{1}+\left[\left\langle\nabla_{s}^{3} \vec{\kappa}_{i}, \nabla_{s}^{4} \vec{\kappa}_{i}\right\rangle\right]_{0}^{1}+\frac{1}{2}\left[\varphi_{i}\left|\nabla_{s}^{2} \vec{\kappa}_{i}\right|^{2}\right]_{0}^{1}\right) \\
\leq \varepsilon \sum_{i=1}^{3} \int_{0}^{1}\left|\nabla_{S}^{4} \vec{\kappa}_{i}\right|^{2} d s+C_{\varepsilon}\left(\lambda_{i}, f_{i, 0}, \mathcal{L}\left(f_{i}\right), \mathcal{E}_{\lambda_{i}}\left(f_{i, 0}\right)\right) .
\end{gathered}
$$

From (6.1), (6.2) and the estimate above choosing $\varepsilon$ appropriately we find that

$$
\sum_{i=1}^{3}\left(\frac{d}{d t} \frac{1}{2} \int_{I}\left|\nabla_{s}^{2} \vec{\kappa}_{i}\right|^{2} d s+\frac{1}{2} \int_{I}\left|\nabla_{s}^{2} \vec{\kappa}_{i}\right|^{2} d s\right) \leq \sum_{i=1}^{3} C\left(\lambda, f_{i, 0}, \mathcal{L}\left(f_{i}\right), \mathcal{E}_{\lambda_{i}}\left(f_{i, 0}\right)\right),
$$

and hence, using the smoothness of the flow and of the initial data, we infer that $\left\|\nabla_{S}^{2} \vec{\kappa}_{i}\right\|_{L^{2}}$ are uniformly bounded on $[0, T)$ for $i=1,2,3$.

Second Step Uniform estimate of $\left\|\nabla_{s}^{6} \vec{\kappa}_{i}\right\|_{L^{2}}$ on $(0, T), i=1,2,3$ for special choice of $\varphi_{i}$

Since equations (1.8) are of fourth order it is natural, after having controlled the second order derivative of the curvature, to control now the sixth order derivative of the curvature and then (later ) in the general step the derivative of order $2+4 m$ for $m \in \mathbb{N}$. With interpolation inequalities we then get also estimates for the intermediate terms.

In this step we proceed as in the previous one applying Lemma 3.2. The crucial point is the choice of the normal vector field $\vec{\phi}$. The main difficulties in the case of networks are the boundary terms and the tangential components. Concerning the boundary terms it is necessary to lower their order (using the boundary conditions). Recall that since the last boundary condition (see (1.9)) involves a sum it was fundamental in (6.3) that $-\nabla_{s}^{2} \vec{\kappa}_{i}$ is equal to the normal component of $\partial_{t} f_{i}$ at the triple junction. In order to use the same idea at this step instead of working with $\nabla_{s}^{6} \vec{\kappa}_{i}$ directly we are going to work with the vector field $\nabla_{t} S_{1, i}$ where

$$
S_{1, i}=-\nabla_{s}^{2} \vec{\kappa}_{i}+\varphi_{i} \partial_{s} f_{i}
$$

that we call speed (motivated by the fact that indeed at the triple junction $S_{1, i}=\partial_{t} f_{i}$ ). This choice is due to the fact that at the triple junction $\partial_{t}^{2} f_{i}=\partial_{t}^{2} f_{j}$. Instead of working with $\partial_{t}^{2} f_{i}$ we can work with shorter expressions by the following considerations. Since in the boundary terms in (3.11) scalar products with normal vector fields of the same curve appear, only the normal component of the vector field is relevant and hence we could work with $\nabla_{t} \partial_{t} f_{i}$. In order to simplify further the computations we use that at the junction point $\partial_{t} f_{i}=-\nabla_{s}^{2} \vec{\kappa}_{i}+\varphi_{i} \partial_{s} f_{i}$ and hence work with $\nabla_{t} S_{1, i}$. As we will see, $\nabla_{t} S_{1, i}$ goes like $-\nabla_{s}^{6} \vec{\kappa}_{i}$ plus lower order terms.

The other difficulty is due to the tangential component characterised by the functions $\varphi_{i}$. These functions are needed to keep the network connected and hence play a role only at the triple junction. Because of this we have informations only at the triple junction (see Remark 5.1 and 5.2), more precisely on $\varphi_{i}(0)$ and $\partial_{t}^{m} \varphi_{i}(0)$ for 
$m \in \mathbb{N}$. On the other hand, when computing the evolution of several quantities, derivatives with respect to $s$ of $\varphi_{i}$ appear. In Section 2.3, in order to simplify the computations, we made a special choice of $\varphi_{i}$ taking the linear interpolation between the value at the junction point, i.e. $\varphi_{i}(0)$, and the other boundary point where $\varphi_{i}(1)=0$ (since the point is kept fixed in time). More precisely, we have choosen to work with

$$
\varphi_{i}(t, x)=\varphi_{i}(t, 0)\left(1-\frac{1}{\mathcal{L}\left(f_{i}\right)} \int_{0}^{x}\left|\partial_{x} f_{i}\right| d x\right), i=1,2,3 .
$$

As a consequence, for $i=1,2,3$ and for any $t \in(0, T)$

$$
\partial_{s} \varphi_{i}(x, t)=-\frac{1}{\mathcal{L}\left(f_{i}\right)} \varphi_{i}(t, 0) \text { and } \partial_{s}^{k} \varphi_{i}(x, t)=0 \text { for } x \in[0,1] \text { for } k \geq 2 .
$$

Moreover using (3.12) we can write

$$
\begin{aligned}
& \partial_{t} \varphi_{i}(t, x)=\partial_{t} \varphi_{i}(t, 0)\left(1-\frac{1}{\mathcal{L}\left(f_{i}\right)} \int_{0}^{x}\left|\left(f_{i}\right)_{x}\right| d x\right)+\frac{\varphi_{i}(t, 0)}{\mathcal{L}\left(f_{i}\right)^{2}}\left(\frac{d}{d t} \mathcal{L}\left(f_{i}\right)\right) \int_{0}^{x}\left|\partial_{x} f_{i}\right| d x \\
& -\frac{\varphi_{i}(t, 0)}{\mathcal{L}\left(f_{i}\right)} \int_{0}^{x}\left\langle\partial_{s} f_{i}, \partial_{x} \partial_{t} f_{i}\right\rangle d x, \\
& =\partial_{t} \varphi_{i}(t, 0)\left(1-\frac{1}{\mathcal{L}\left(f_{i}\right)} \int_{0}^{x}\left|\partial_{x} f_{i}\right| d x\right)-\frac{\varphi_{i}(t, 0)}{\mathcal{L}\left(f_{i}\right)^{2}}\left(\varphi_{i}(t, 0)+\int_{I}\left\langle\vec{\kappa}_{i}, \vec{V}_{i}\right\rangle d s\right) \int_{0}^{x}\left|\partial_{x} f_{i}\right| d x \\
& -\frac{\varphi_{i}(t, 0)}{\mathcal{L}\left(f_{i}\right)} \int_{0}^{x}\left(\partial_{s} \varphi_{i}-\left\langle\vec{\kappa}_{i}, \vec{V}_{i}\right\rangle\right) d s .
\end{aligned}
$$

From here on we will use these special choices of $\varphi_{i}$ 's without further notice. Observe that in the first step $\varphi_{i}$ were arbitrary.

We can now start with the computations. By Lemma 3.2 with $\vec{\phi}=\nabla_{t} S_{1, i}$ and summing over $i$ we find

$$
\begin{aligned}
& \frac{d}{d t} \sum_{i=1}^{3} \frac{1}{2} \int_{I}\left|\nabla_{t} S_{1, i}\right|^{2} d s+\sum_{i=1}^{3} \int_{I}\left|\nabla_{s}^{2} \nabla_{t} S_{1, i}\right|^{2} d s \\
& =-\sum_{i=1}^{3}\left[\left\langle\nabla_{t} S_{1, i}, \nabla_{s}^{3} \nabla_{t} S_{1, i}\right\rangle\right]_{0}^{1}+\sum_{i=1}^{3}\left[\left\langle\nabla_{s} \nabla_{t} S_{1, i}, \nabla_{s}^{2} \nabla_{t} S_{1, i}\right\rangle\right]_{0}^{1} \\
& \quad+\sum_{i=1}^{3} \int_{I}\left\langle\left(\nabla_{t}+\nabla_{s}^{4}\right) \nabla_{t} S_{1, i}+\frac{1}{2} \nabla_{t} S_{1, i} \partial_{s} \varphi_{i}, \nabla_{t} S_{1, i}\right\rangle d s-\frac{1}{2} \sum_{i=1}^{3} \int_{I}\left|\nabla_{t} S_{1, i}\right|^{2}\left\langle\vec{\kappa}_{i}, \vec{V}_{i}\right\rangle d s .
\end{aligned}
$$

We need to compute these terms. Since

$$
\vec{V}_{i}=\sum_{\substack{[[a, b]] \leq[[2,1]] \\ c \leq 2, b \text { odd }}} P_{b}^{a, c}\left(\vec{\kappa}_{i}\right), \quad\left\langle\vec{\kappa}_{i}, \vec{V}_{i}\right\rangle=\sum_{\substack{[[a, b]] \leq[[2,2]] \\ c \leq 2, \text { b even }}} P_{b}^{a, c}\left(\vec{\kappa}_{i}\right),
$$

by Lemma 3.5 (with $m=2$ and $m=6$ ), (3.3), (3.2), (3.1), (3.6), (6.7), (3.16), (3.9) we find

$$
h:=\varphi_{i}^{2}, \quad \partial_{s} h=2 \varphi_{i} \partial_{s} \varphi_{i}, \quad \partial_{s}^{2} h=2\left(\partial_{s} \varphi_{i}\right)^{2}, \quad \partial_{s}^{m} h=0 \text { for } m \geq 3,
$$

and

$$
\nabla_{t} S_{1, i}=\nabla_{s}^{6} \vec{\kappa}_{i}+\sum_{\substack{[[a, b]] \leq[[4,3]] \\ c \leq 4, b \text { odd }}} P_{b}^{a, c}\left(\vec{\kappa}_{i}\right)+\varphi_{i} \sum_{\substack{[[a, b]] \leq[[3,1]] \\ c \leq 3, b \text { odd }}} P_{b}^{a, c}\left(\vec{\kappa}_{i}\right)+\varphi_{i}^{2} \vec{\kappa}_{i},
$$




$$
\begin{aligned}
& \nabla_{s}^{2} \nabla_{t} S_{1, i}=\nabla_{s}^{8} \vec{\kappa}_{i}+\sum_{\substack{[[a, b]] \leq[[6,3]] \\
c \leq 6, b \text { odd }}} P_{b}^{a, c}\left(\vec{\kappa}_{i}\right)+\varphi_{i} \sum_{\begin{array}{c}
{[[a, b]] \leq \leq[[5,1]]} \\
c \leq 5, b \text { odd }
\end{array}} P_{b}^{a, c}\left(\vec{\kappa}_{i}\right) \\
& +\partial_{s} \varphi_{i} \sum_{\substack{[[a, b]] \leq[[4,1]] \\
c \leq 4, b \text { odd }}} P_{b}^{a, c}\left(\vec{\kappa}_{i}\right)+\varphi_{i}^{2} \nabla_{s}^{2} \vec{\kappa}_{i}+4 \varphi_{i} \partial_{s} \varphi_{i} \nabla_{s} \vec{\kappa}_{i}+2\left(\partial_{s} \varphi_{i}\right)^{2} \vec{\kappa}_{i}, \\
& \nabla_{s}^{4} \nabla_{t} S_{1, i}=\nabla_{s}^{10} \vec{\kappa}_{i}+\sum_{\substack{[[a, b]] \leq[[8,3]] \\
c \leq 8, b \text { odd }}} P_{b}^{a, c}\left(\vec{\kappa}_{i}\right)+\varphi_{i} \sum_{\begin{array}{c}
{[[a, b]] \leq[[7,1]]} \\
c \leq 7, b \text { odd }
\end{array}} P_{b}^{a, c}\left(\vec{\kappa}_{i}\right) \\
& +\partial_{s} \varphi_{i} \sum_{\substack{[[a, b]] \leq[[6,1]] \\
c \leq 6, b \text { odd }}} P_{b}^{a, c}\left(\vec{\kappa}_{i}\right)+\varphi_{i}^{2} \nabla_{s}^{4} \vec{\kappa}_{i}+8 \varphi_{i} \partial_{s} \varphi_{i} \nabla_{s}^{3} \vec{\kappa}_{i}+12\left(\partial_{s} \varphi_{i}\right)^{2} \nabla_{s}^{2} \vec{\kappa}_{i}, \\
& \nabla_{t} \nabla_{t} S_{1, i}=-\nabla_{s}^{10} \vec{\kappa}_{i}+\sum_{\substack{[[a, b]] \leq \leq[8,3]] \\
c \leq 8, b \text { odd }}} P_{b}^{a, c}\left(\vec{\kappa}_{i}\right)+\varphi_{i} \sum_{\begin{array}{c}
{[[a, b]] \leq[[7,1]]} \\
c \leq 7, b \text { odd }
\end{array}} P_{b}^{a, c}\left(\vec{\kappa}_{i}\right) \\
& +\partial_{t} \varphi_{i} \sum_{\substack{[[a, b]] \leqslant \leq[3,1]] \\
c \leq 3, b \text { odd }}} P_{b}^{a, c}\left(\vec{\kappa}_{i}\right)+\varphi_{i}^{2} \sum_{\substack{[[a, b]] \leqslant[[4,1]] \\
c \leq 4, b \text { odd }}} P_{b}^{a, c}\left(\vec{\kappa}_{i}\right)+\varphi_{i}^{3} \nabla_{s} \vec{\kappa}_{i}+2 \varphi_{i} \partial_{t} \varphi_{i} \vec{\kappa}_{i} .
\end{aligned}
$$

Due to our choice of $\varphi_{i}$ and since the length is uniformly bounded from below, we have (up to a constant) the same estimate from above for $\left|\varphi_{i}\right|$ and $\left|\partial_{s} \varphi_{i}\right|$, namely

$$
\left|\varphi_{i}(t, x)\right| \leq|\varphi(t, 0)|:=\left|\varphi_{i}(0)\right| \text { and }\left|\partial_{s} \varphi_{i}(t, x)\right| \leq C\left|\varphi_{i}(0)\right| \text { for all } x \in[0,1] .
$$

Also from (6.8), the uniform bounds for the curvature derived in the first step, and interpolation inequalities we infer

$$
\left|\partial_{t} \varphi_{i}(t, x)\right| \leq\left|\partial_{t} \varphi_{i}(t, 0)\right|+C\left(\left|\varphi_{i}(t, 0)\right|^{2}+\left|\varphi_{i}(t, 0)\right|\right)=\left|\partial_{t} \varphi_{i}(0)\right|+C\left(\left|\varphi_{i}(0)\right|^{2}+\left|\varphi_{i}(0)\right|\right) .
$$

As a consequence we find on $[0,1]$

$$
\begin{aligned}
\left|\left(\nabla_{t}+\nabla_{s}^{4}\right) \nabla_{t} S_{1, i}\right| \leq & \sum_{\substack{[[a, b]] \leq \leq[8,3]] \\
c \leq 8, b \text { odd }}}\left|P_{b}^{a, c}\left(\vec{\kappa}_{i}\right)\right|+\left|\varphi_{i}(0)\right| \sum_{\substack{[[a, b]] \leq[[7,1]] \\
c \leq 7, b \text { odd }}}\left|P_{b}^{a, c}\left(\vec{\kappa}_{i}\right)\right| \\
& +\left|\varphi_{i}(0)\right|^{2} \sum_{\substack{[[a, b]] \leq[[4,1]] \\
c \leq 4, b \text { odd }}}\left|P_{b}^{a, c}\left(\vec{\kappa}_{i}\right)\right|+\left|\varphi_{i}(0)\right|^{3} \sum_{\substack{[[a, b]] \leq \leq[1,1]] \\
c \leq 1, b \text { odd }}}\left|P_{b}^{a, c}\left(\vec{\kappa}_{i}\right)\right| \\
& +\left|\partial_{t} \varphi_{i}(0)\right| \sum_{\substack{[[a, b]] \leq[[3,1]] \\
c \leq 3, b \text { odd }}}\left|P_{b}^{a, c}\left(\vec{\kappa}_{i}\right)\right|+2\left|\varphi_{i}(0) \partial_{t} \varphi_{i}(0)\right|\left|\vec{\kappa}_{i}\right| .
\end{aligned}
$$

Next, using the simple inequalities

$$
|a+b|^{2}=|a|^{2}+|b|^{2}+2 a \cdot b \geq(1-\epsilon)|a|^{2}-C_{\epsilon}\left|b^{2}\right|, \quad\left(\sum_{i=1}^{m} a_{i}\right)^{2} \leq C(m)\left(\sum_{i=1}^{m} a_{i}^{2}\right)
$$

and (6.11) we infer that (for instance use the expressions derived above in (6.10) to write $\nabla_{S}^{2} \nabla_{t} S_{1, i}=\nabla_{S}^{8} \vec{\kappa}_{i}+$ rest and take $a=\nabla_{s}^{8} \vec{\kappa}_{i}, b=$ rest and $\epsilon=\frac{1}{2}$, then use that rest $=\sum \ldots$ to evaluate the $b$-term)

$$
\begin{aligned}
\left|\nabla_{S}^{2} \nabla_{t} S_{1, i}\right|^{2} \geq & \frac{1}{2}\left|\nabla_{S}^{8} \vec{\kappa}_{i}\right|^{2}-\sum_{\substack{[[a, b]] \leq[[12,6]] \\
c \leq 6, b \text { even }}}\left|P_{b}^{a, c}\left(\vec{\kappa}_{i}\right)\right| \\
& -\left|\varphi_{i}(0)\right|^{2} \sum_{\substack{[[a, b]] \leq[[10,2]] \\
c \leq 5, \text { beven }}}\left|P_{b}^{a, c}\left(\vec{\kappa}_{i}\right)\right|-\left|\varphi_{i}(0)\right|^{4} \sum_{\substack{[[a, b]] \leq[[4,2]] \\
c \leq 2, b \text { even }}}\left|P_{b}^{a, c}\left(\vec{\kappa}_{i}\right)\right|
\end{aligned}
$$

as well as

$$
\left|\nabla_{t} S_{1, i}\right|^{2} \geq \frac{1}{2}\left|\nabla_{s}^{6} \vec{\kappa}_{i}\right|^{2}-\sum_{\substack{[[a, b]] \leq[[8,6]] \\ c \leq 4, b \text { even }}}\left|P_{b}^{a, c}\left(\vec{\kappa}_{i}\right)\right|
$$




$$
-\left|\varphi_{i}(0)\right|^{2} \sum_{\substack{[[a, b]] \leq[[6,2]] \\ c \leq 3, b \text { even }}}\left|P_{b}^{a, c}\left(\vec{\kappa}_{i}\right)\right|-\left|\varphi_{i}(0)\right|^{4} \sum_{\substack{[[a, b]] \leq[[0,2]] \\ c \leq 0, b \text { even }}}\left|P_{b}^{a, c}\left(\vec{\kappa}_{i}\right)\right| .
$$

From (6.9), adding $\sum_{i=1}^{3} \frac{1}{2} \int_{I}\left|\nabla_{t} S_{1, i}\right|^{2} d s$ to both sides of the equation, we get using the previous relations that

$$
\begin{aligned}
& \frac{d}{d t} \sum_{i=1}^{3} \frac{1}{2} \int_{I}\left|\nabla_{t} S_{1, i}\right|^{2} d s+\sum_{i=1}^{3} \frac{1}{2} \int_{I}\left|\nabla_{t} S_{1, i}\right|^{2} d s+\sum_{i=1}^{3} \frac{1}{2} \int_{I}\left|\nabla_{s}^{8} \vec{\kappa}_{i}\right|^{2} d s \\
& \leq-\sum_{i=1}^{3}\left[\left\langle\nabla_{t} S_{1, i}, \nabla_{s}^{3} \nabla_{t} S_{1, i}\right\rangle\right]_{0}^{1}+\sum_{i=1}^{3}\left[\left\langle\nabla_{s} \nabla_{t} S_{1, i}, \nabla_{s}^{2} \nabla_{t} S_{1, i}\right\rangle\right]_{0}^{1} \\
& \quad+\sum_{i=1}^{3} \int_{I} \sum_{\substack{[[a, b]] \leq[[14,4]] \\
c \leq 8, \text { beven }}}\left|P_{b}^{a, c}\left(\vec{\kappa}_{i}\right)\right| d s+\sum_{i=1}^{3} \sum_{j=1}^{5}\left|\varphi_{i}(0)\right|^{j} \int_{I} \sum_{\substack{[[a, b]] \leq[[16-3 j, 2]] \\
c \leq \min \{8, a\}, b \text { even }}}\left|P_{b}^{a, c}\left(\vec{\kappa}_{i}\right)\right| d s \\
& \quad+\sum_{i=1}^{3} \sum_{j=0}^{3}\left|\partial_{t} \varphi_{i}(0) \| \varphi_{i}(0)\right|^{j} \int_{I}^{\substack{[[a, b]] \leq[[9-3 j, 2]] \\
c \leq \min \{6, a\}, \text { beven }}}\left|P_{b}^{a, c}\left(\vec{\kappa}_{i}\right)\right| d s .
\end{aligned}
$$

We first estimate the integral terms. By Lemma 3.11 (more precisely, (3.27) with $A=14, B=4, c \leq 8, \ell=6$ ) one sees that

$$
\sum_{i=1}^{3} \int_{\substack { I \\
\begin{subarray}{c}{[a, b]] \leq[114,4]] \\
c \leq 8, b \text { even }{ I \\
\begin{subarray} { c } { [ a , b ] ] \leq [ 1 1 4 , 4 ] ] \\
c \leq 8 , b \text { even } } }\end{subarray}}\left|P_{b}^{a, c}\left(\vec{\kappa}_{i}\right)\right| \leq \varepsilon \sum_{i=1}^{3} \int_{0}^{1}\left|\nabla_{s}^{8} \vec{\kappa}_{i}\right|^{2} d s+C_{\epsilon}\left(\lambda_{i}, f_{i, 0}, \mathcal{L}\left(f_{i}\right), \varepsilon_{\lambda_{i}}\left(f_{i, 0}\right)\right),
$$

with $\varepsilon \in(0,1)$. Here we have used the fact that the length of the curves remains bounded away from zero. Again by Lemma 3.11 (now using (3.26) but still with $\ell=6$ ) and (5.13) we get

$$
\sum_{j=1}^{5}\left|\varphi_{i}(0)\right|^{j} \sum_{i=1}^{3} \int_{I} \sum_{\substack{[[a, b]] \leq[[16-3 j, 2]] \\ c \leq \min \{8, a\}, b \text { even }}}\left|P_{b}^{a, c}\left(\vec{\kappa}_{i}\right)\right| \leq \frac{C}{\delta} \sum_{j=1}^{5} \sum_{i=1}^{3} \sum_{k=1}^{3}\left\|\vec{\kappa}_{k}\right\|_{8,2}^{\frac{5 j}{16}} \max \left\{1,\left\|\vec{\kappa}_{i}\right\|_{8,2}\right\}^{\frac{16-3 j}{8}} .
$$

Then using Corollary 3.9, the uniform bound on the lengths and Lemma A.1 we find

$$
\sum_{j=1}^{5}\left|\varphi_{i}(0)\right|^{j} \sum_{i=1}^{3} \int_{I} \sum_{\substack{[[a, b]] \leq[[16-3 j, 2]] \\ c \leq \min \{8, a\}, b \text { even }}}\left|P_{b}^{a, c}\left(\vec{\kappa}_{i}\right)\right| \leq \varepsilon \sum_{i=1}^{3} \int_{0}^{1}\left|\nabla_{s}^{8} \vec{\kappa}_{i}\right|^{2} d s+C_{\epsilon},
$$

where $C_{\epsilon}=C_{\epsilon}\left(\delta, \lambda_{i}, f_{i, 0}, \mathcal{L}\left(f_{i}\right), \mathcal{E}_{\lambda_{i}}\left(f_{i, 0}\right)\right)$. With the same arguments and now using also (5.14) we estimate

$$
\begin{aligned}
& \sum_{i=1}^{3} \sum_{j=0}^{3}\left|\partial_{t} \varphi_{i}(0) \| \varphi_{i}(0)\right|^{j} \int_{I} \sum_{\substack{[[a, b]] \leq[9-3 j, 2]] \\
c \leq \min \{6, a\}, b \text { even }}}\left|P_{b}^{a, c}\left(\vec{\kappa}_{i}\right)\right| d s \\
& \leq \frac{C}{\delta^{2}} \sum_{i=1}^{3} \sum_{j=0}^{3} \sum_{k=1}^{3} \max \left\{1,\left\|\vec{\kappa}_{k}\right\|_{8,2}\right\}^{\frac{13}{16}} \sum_{l=1}^{3}\left\|\vec{\kappa}_{l}\right\|_{8,2}^{\frac{5 j}{16}} \max \left\{1,\left\|\vec{\kappa}_{i}\right\|_{8,2}\right\}^{\frac{9-3 j}{8}} \\
& \leq \varepsilon \sum_{i=1}^{3} \int_{0}^{1}\left|\nabla_{s}^{8} \vec{\kappa}_{i}\right|^{2} d s+C_{\epsilon}\left(\delta, \lambda_{i}, f_{i, 0}, \mathcal{L}\left(f_{i}\right), \mathcal{E}_{\lambda_{i}}\left(f_{i, 0}\right)\right),
\end{aligned}
$$

using again the general Young inequality of Lemma A.1. It remains to treat the boundary terms. We may write

$$
-\sum_{i=1}^{3}\left[\left\langle\nabla_{t} S_{1, i}, \nabla_{s}^{3} \nabla_{t} S_{1, i}\right\rangle\right]_{0}^{1}=-\sum_{i=1}^{3}\left[\left\langle\partial_{t} S_{1, i}, \nabla_{s}^{3} \nabla_{t} S_{1, i}\right\rangle\right]_{0}^{1}
$$


Since $S_{1, i}=\partial_{t} f_{i}$ at the boundary points, the velocities and their time derivatives coincide at the boundary points (and vanish at $x=1$ where the points are fixed in time) we find

$$
-\sum_{i=1}^{3}\left[\left\langle\nabla_{t} S_{1, i}, \nabla_{S}^{3} \nabla_{t} S_{1, i}\right\rangle\right]_{0}^{1}=\left.\left\langle\partial_{t} S_{1,1}, \sum_{i=1}^{3} \nabla_{S}^{3} \nabla_{t} S_{1, i}\right\rangle\right|_{x=0} .
$$

Since

$$
\begin{aligned}
\nabla_{S}^{3} \nabla_{t} S_{1, i}= & \nabla_{s}^{9} \vec{\kappa}_{i}+\sum_{\substack{[[a, b]] \leq[[7,3]] \\
c \leq 7, b \text { odd }}} P_{b}^{a, c}\left(\vec{\kappa}_{i}\right)+\varphi_{i} \sum_{\substack{[[a, b]] \leq[[6,1]] \\
c \leq 6, b \text { odd }}} P_{b}^{a, c}\left(\vec{\kappa}_{i}\right) \\
& +\partial_{s} \varphi_{i} \sum_{\substack{[[a, b]] \leq[[5,1]] \\
c \leq 5, b \text { odd }}} P_{b}^{a, c}\left(\vec{\kappa}_{i}\right)+\varphi_{i}^{2} \nabla_{s}^{3} \vec{\kappa}_{i}+6 \varphi_{i} \partial_{s} \varphi_{i} \nabla_{s}^{2} \vec{\kappa}_{i}+6\left(\partial_{s} \varphi_{i}\right)^{2} \nabla_{s} \vec{\kappa}_{i},
\end{aligned}
$$

by (6.10), we get using (4.6) the following order reduction

$$
\begin{aligned}
\sum_{i=1}^{3} \nabla_{s}^{3} \nabla_{t} S_{1, i}= & \sum_{i=1}^{3}\left(\sum_{\substack{[[a, b]] \leq[[7,3]] \\
c \leq 7, b \text { odd }}} P_{b}^{a, c}\left(\vec{\kappa}_{i}\right)+\varphi_{i} \sum_{\substack{[[a, b]] \leq[[6,1]] \\
c \leq 6, b \text { odd } \\
b}} P_{b}^{a, c}\left(\vec{\kappa}_{i}\right)\right. \\
& +\partial_{s} \varphi_{i} \sum_{\substack{[[a, b]] \leq[[5,1]] \\
c \leq 5, b \text { odd }}} P_{b}^{a, c}\left(\vec{\kappa}_{i}\right)+6 \varphi_{i} \partial_{s} \varphi_{i} \nabla_{s}^{2} \vec{\kappa}_{i}+6\left(\partial_{s} \varphi_{i}\right)^{2} \nabla_{s} \vec{\kappa}_{i} \\
& \left.-\partial_{t} \varphi_{i} \nabla_{s}^{2} \vec{\kappa}_{i}+\partial_{s} f_{i}\left(\sum_{\substack{[[a, b]] \leq[[8,2]] \\
c \leq 7, b \text { even }}} P_{b}^{a, c}\left(\vec{\kappa}_{i}\right)+\varphi_{i} \sum_{\substack{[[a, b]] \leq[[5,2]] \\
c \leq 4, b \text { even }}} P_{b}^{a, c}\left(\vec{\kappa}_{i}\right)\right)\right)\left.\right|_{x=0} .
\end{aligned}
$$

Note that we can write the first term as

$$
\sum_{\substack{[[a, b]] \leq[[7,3]] \\ c \leq 7, b \text { odd }}} P_{b}^{a, c}\left(\vec{\kappa}_{i}\right)=\sum_{\substack{[[a, b]] \leq[[7,3]] \\ c \leq 7, b \text { odd } b \neq 1}} P_{b}^{a, c}\left(\vec{\kappa}_{i}\right)+\sum_{\substack{[[a, b]] \leq[[7,3]] \\ c \leq 7, b=1}} P_{b}^{a, c}\left(\vec{\kappa}_{i}\right)
$$

and by the embedding estimate (3.23) (applied to each term in the sum) we can write

$$
\left|\sum_{\substack{[[a, b]] \leq[[7,3]] \\ c \leq 7, b \text { odd } b \neq 1}} P_{b}^{a, c}\left(\vec{\kappa}_{i}\right)\right| \leq \int_{I} \sum_{\substack{[[a, b]] \leq[[8,3]] \\ c \leq 8, b \geq 2}}\left|P_{b}^{a, c}\left(\vec{\kappa}_{i}\right)\right| d s .
$$

Then using Lemma 3.7 for the other terms and (6.11) we obtain

$$
\begin{aligned}
& \left|\sum_{i=1}^{3} \nabla_{s}^{3} \nabla_{t} S_{1, i}\right|_{x=0} \leq \sum_{i=1}^{3} \int_{I} \sum_{\substack{[[a, b]] \leq[[8,3]] \\
c \leq 8, b \geq 2}}\left|P_{b}^{a, c}\left(\vec{\kappa}_{i}\right)\right| d s
\end{aligned}
$$

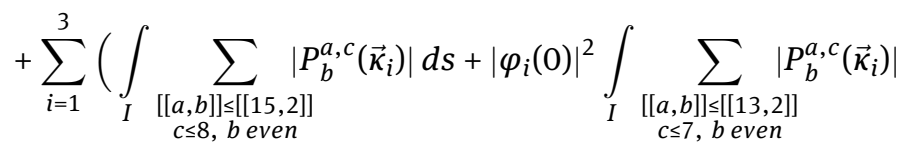

$$
\begin{aligned}
& \left.+\left|\varphi_{i}(0)\right|^{4} \int_{I} \sum_{\substack{[[a, b]] \leq[[7,2]] \\
c \leq 4, b \text { even }}}\left|P_{b}^{a, c}\left(\vec{\kappa}_{i}\right)\right| d s\right)^{\frac{1}{2}} \\
& +\sum_{i=1}^{3}\left(\int_{I} \sum_{\substack{[[a, b]] \leq[[9,2]] \\
c \leq 8, b \text { even }}}\left|P_{b}^{a, c}\left(\vec{\kappa}_{i}\right)\right| d s+\left|\varphi_{i}(0)\right| \int_{I} \sum_{\substack{[[a, b]] \leq[[6,2]] \\
c \leq 5, \text { beven }}}\left|P_{b}^{a, c}\left(\vec{\kappa}_{i}\right)\right| d s\right) \\
& +\sum_{i=1}^{3}\left|\partial_{t} \varphi_{i}(0)\right|\left|\nabla_{s}^{2} \vec{\kappa}_{i}\right|
\end{aligned}
$$


By Lemma 3.11 and (5.13), again with $\ell=6$, with the same arguments as above we see that the integrals on the right hand side can be bounded by

$$
\begin{aligned}
\left|\sum_{i=1}^{3} \nabla_{S}^{3} \nabla_{t} S_{1, i}\right|_{x=0} \leq & C \sum_{i=1}^{3} \max \left\{1,\left\|\vec{\kappa}_{i}\right\|_{8,2}\right\}^{\frac{18}{16}}+\frac{C}{\delta} \sum_{i=1}^{3} \sum_{k=1}^{3}\left\|\vec{\kappa}_{k}\right\|_{8,2}^{\frac{5}{16}} \max \left\{1,\left\|\vec{\kappa}_{i}\right\|_{8,2}\right\}^{\frac{13}{16}} \\
& +\frac{C}{\delta^{2}} \sum_{i=1}^{3} \sum_{k=1}^{3}\left\|\vec{\kappa}_{k}\right\|_{8,2}^{\frac{5}{8}} \max \left\{1,\left\|\vec{\kappa}_{i}\right\|_{8,2}\right\}^{\frac{7}{16}}+\sum_{i=1}^{3} \mid \partial_{t} \varphi_{i}(0)\left\|\nabla_{S}^{2} \vec{\kappa}_{i}\right\|_{x=0} .
\end{aligned}
$$

For the last term we observe that since $\vec{\kappa}_{i}(1)=0$ and $\partial_{t} f(1)=0$ it follows $\nabla_{s}^{2} \vec{\kappa}_{i}(1)=0$ and hence with Lemma 3.11

$$
\left|\nabla_{S}^{2} \vec{\kappa}_{i}(0)\right|=\left.|| \nabla_{s}^{2} \vec{\kappa}_{i}(1)\right|^{2}-\left.\left|\nabla_{S}^{2} \vec{\kappa}_{i}(0)\right|^{2}\right|^{\frac{1}{2}}=\left(2 \int_{I}\left\langle\nabla_{S}^{2} \vec{\kappa}_{i}, \nabla_{S}^{3} \vec{\kappa}_{i}\right\rangle d s\right)^{\frac{1}{2}} \leq C \max \left\{1,\left\|\vec{\kappa}_{i}\right\|_{8,2}\right\}^{\frac{5}{16}} .
$$

By (5.14) we finally get

$$
\left|\partial_{t} \varphi_{i}(0)\right|\left|\nabla_{S}^{2} \vec{\kappa}_{i}\right|_{x=0} \leq \frac{C}{\delta} \sum_{j=1}^{3} \max \left\{1,\left\|\vec{\kappa}_{j}\right\|_{8,2}\right\}^{\frac{13}{16}} \max \left\{1,\left\|\vec{\kappa}_{i}\right\|_{8,2}\right\}^{\frac{5}{16}} .
$$

On the other hand with (3.7), Lemma 3.5, (3.6), and using that $\vec{\kappa}_{i}=0$ at the boundary we find

$$
\begin{aligned}
\partial_{t} S_{1, i}(0)= & \nabla_{s}^{6} \vec{\kappa}_{i}+\sum_{\substack{[[a, b]] \leq \leq[[4,3]] \\
c \leq 4, \text { bodd }}} P_{b}^{a, c}\left(\vec{\kappa}_{i}\right)+\varphi_{i} \sum_{\substack{[[a, b]] \leq[[3,1]] \\
c \leq 3, b \text { odd }}} P_{b}^{a, c}\left(\vec{\kappa}_{i}\right) \\
& +\partial_{t} \varphi_{i} \partial_{s} f_{i}+\partial_{s} f_{i} \sum_{\substack{[[a, b]] \leq[[[5,2]] \\
c \leq 3, \text { beven }}} P_{b}^{a, c}\left(\vec{\kappa}_{i}\right),
\end{aligned}
$$

so that with Lemma 3.7, Lemma 3.11, (5.13) and (5.14) we obtain

$$
\begin{aligned}
\left|\partial_{t} S_{1, i}\right|(0) \leq & \left(\int_{I} \sum_{\substack{[[a, b]] \leq[[13,2]] \\
c \leq 7, b \text { beven }}}\left|P_{b}^{a, c}\left(\vec{\kappa}_{i}\right)\right| d s+\left|\varphi_{i}(0)\right|^{2} \int_{I} \sum_{\substack{[[a, b]] \leq[[7,2]] \\
c \leq 4, b \text { odd }}}\left|P_{b}^{a, c}\left(\vec{\kappa}_{i}\right)\right| d s\right)^{\frac{1}{2}} \\
& +\sum_{\substack{[[a, b]] \leq \leq[[6,2]] \\
c \leq 4, \text { beven }}} \int_{I}\left|P_{b}^{a, c}\left(\vec{\kappa}_{i}\right)\right| d s+\left|\partial_{t} \varphi_{i}(0)\right| \\
\leq & C \max \left\{1,\left\|\vec{\kappa}_{i}\right\|_{8,2}\right\}^{\frac{13}{16}}+\frac{C}{\delta}\left(\sum_{k=1}^{3}\left\|\vec{\kappa}_{k}\right\|_{8,2}^{\frac{5}{16}} \max \left\{1,\left\|\vec{\kappa}_{i}\right\|_{8,2}\right\}^{\frac{7}{16}}+\max \left\{1,\left\|\vec{\kappa}_{i}\right\|_{8,2}\right\}^{\frac{13}{16}}\right) .
\end{aligned}
$$

Since the sum of the exponents of the several terms is smaller than 2 we can use Lemma A.1 and we obtain

$$
\left|\sum_{i=1}^{3}\left[\left\langle\nabla_{t} S_{1, i}, \nabla_{S}^{3} \nabla_{t} S_{1, i}\right\rangle\right]_{0}^{1}\right| \leq \varepsilon \sum_{i=1}^{3} \int_{0}^{1}\left|\nabla_{s}^{8} \vec{\kappa}_{i}\right|^{2} d s+C_{\epsilon}\left(\delta, \lambda_{i}, f_{i, 0}, \mathcal{L}\left(f_{i}\right), \varepsilon_{\lambda_{i}}\left(f_{i, 0}\right)\right),
$$

with $\varepsilon \in(0,1)$.

It remains to evaluate the last boundary term $\sum_{i=1}^{3}\left[\left\langle\nabla_{s} \nabla_{t} S_{1, i}, \nabla_{s}^{2} \nabla_{t} S_{1, i}\right\rangle\right]_{0}^{1}$ in (6.9). First of all note that by (6.10), the fact that $\vec{\kappa}_{i}=0$, (4.2) (at $x=0$ ) respectively Lemma 4.1 ( at $x=1$ ), we infer that at the boundary points we have (since there are some cancellations and $\vec{\kappa}_{i}=0$ )

$$
\begin{aligned}
\nabla_{s}^{2} \nabla_{t} S_{1, i}= & \sum_{\substack{[[a, b]] \leq[[6,3]] \\
c \leq 6, b \text { odd }}} P_{b}^{a, c}\left(\vec{\kappa}_{i}\right)+\varphi_{i} \sum_{\substack{[[a, b]] \leq[[5,1]] \\
c \leq 5, b \text { odd }}} P_{b}^{a, c}\left(\vec{\kappa}_{i}\right)+\partial_{s} \varphi_{i} \sum_{\substack{[[a, b]] \leq[[4,1]] \\
c \leq 4, \text { bodd }}} P_{b}^{a, c}\left(\vec{\kappa}_{i}\right) \\
& +4 \varphi_{i} \partial_{s} \varphi_{i} \nabla_{s} \vec{\kappa}_{i}-\partial_{t} \varphi_{i} \nabla_{s} \vec{\kappa}_{i},
\end{aligned}
$$


and

$$
\begin{aligned}
\nabla_{s} \nabla_{t} S_{1, i}= & \nabla_{s}^{7} \vec{\kappa}_{i}+\sum_{\substack{[[a, b]] \leq[[5,3]] \\
c \leq 5, b \text { odd }}} P_{b}^{a, c}\left(\vec{\kappa}_{i}\right)+\partial_{s} \varphi_{i} \sum_{\substack{[[a, b]] \leq[[3,1]] \\
c \leq 3, b \text { odd }}} P_{b}^{a, c}\left(\vec{\kappa}_{i}\right) \\
& +\varphi_{i} \sum_{\substack{[[a, b]] \leq[[4,1]] \\
c \leq 4, b \text { odd }}} P_{b}^{a, c}\left(\vec{\kappa}_{i}\right)+\varphi_{i}^{2} \nabla_{s} \vec{\kappa}_{i} .
\end{aligned}
$$

We estimate each term separately at the boundary $x=0$ and $x=1$. (At $x=1$ many terms do actually vanish since here $\partial_{t} \varphi_{i}=0=\varphi_{i}$, however we do not distinguish the treatment of the terms.) We use Lemma 3.7, (6.7), Lemma 3.11, (5.13), (5.14) to obtain at $x=0$ or $x=1$

$$
\begin{aligned}
& \left|\nabla_{s}^{2} \nabla_{t} S_{1, i}\right| \leq\left(\int_{I} \sum_{\substack{[[a, b]] \leq[[13,6]] \\
c \leq 7, b \text { even }}}\left|P_{b}^{a, c}\left(\vec{\kappa}_{i}\right)\right| d s+\left|\varphi_{i}(0)\right|^{2} \int_{I} \sum_{\begin{array}{c}
[[a, b]] \leq[111,2]] \\
c \leq 6, \text { beven }
\end{array}}\left|P_{b}^{a, c}\left(\vec{\kappa}_{i}\right)\right| d s\right. \\
& \left.+\left|\varphi_{i}(0)\right|^{4} \int_{I} \sum_{\substack{[[a, b]] \leq[[3,2]] \\
c \leq 2, b \text { even }}}\left|P_{b}^{a, c}\left(\vec{\kappa}_{i}\right)\right| d s\right)^{\frac{1}{2}}+\left|\partial_{t} \varphi_{i}(0)\right|\left(\int_{I} \sum_{\substack{[[a, b]] \leq[[3,2]] \\
c \leq 2, b \text { even }}}\left|P_{b}^{a, c}\left(\vec{\kappa}_{i}\right)\right| d s\right)^{\frac{1}{2}} \\
& \leq C \max \left\{1,\left\|\vec{\kappa}_{i}\right\|_{8,2}\right\}^{\frac{15}{16}}+\frac{C}{\delta} \sum_{k=1}^{3}\left\|\vec{\kappa}_{k}\right\|_{8,2}^{\frac{5}{16}} \max \left\{1,\left\|\vec{\kappa}_{i}\right\|_{8,2}\right\}^{\frac{11}{16}} \\
& +\frac{C}{\delta^{2}} \sum_{k=1}^{3}\left\|\vec{\kappa}_{k}\right\|_{8,2}^{\frac{10}{16}} \max \left\{1,\left\|\vec{\kappa}_{i}\right\|_{8,2}\right\}^{\frac{3}{16}}+\frac{C}{\delta} \sum_{k=1}^{3}\left\|\vec{\kappa}_{k}\right\|_{8,2}^{\frac{13}{16}} \max \left\{1,\left\|\vec{\kappa}_{i}\right\|_{8,2}\right\}^{\frac{3}{16}},
\end{aligned}
$$

and similarly

$$
\begin{aligned}
\left|\nabla_{s} \nabla_{t} S_{1, i}\right| \leq & \left(\int_{I} \sum_{\substack{[[a, b]] \leq[115,2]] \\
c \leq 8, \text { beven }}}\left|P_{b}^{a, c}\left(\vec{\kappa}_{i}\right)\right| d s+\left|\varphi_{i}(0)\right|^{2} \int_{I} \sum_{\substack{[a, b]] \leq[[9,2]] \\
c \leq 5, \text { beven }}}\left|P_{b}^{a, c}\left(\vec{\kappa}_{i}\right)\right| d s\right. \\
& \left.+\left|\varphi_{i}(0)\right|^{4} \int_{I} \sum_{\substack{[[a, b]] \leq \leq[3,2]] \\
c \leq 2, b \text { even }}}\left|P_{b}^{a, c}\left(\vec{\kappa}_{i}\right)\right| d s\right)^{\frac{1}{2}} \\
\leq & C \max \left\{1,\left\|\vec{\kappa}_{i}\right\|_{8,2}\right\}^{\frac{15}{16}}+\frac{C}{\delta} \sum_{k=1}^{3}\left\|\vec{\kappa}_{k}\right\|_{8,2}^{\frac{5}{16}} \max \left\{1,\left\|\vec{\kappa}_{i}\right\|_{8,2}\right\}^{\frac{9}{16}} \\
& +\frac{C}{\delta^{2}} \sum_{k=1}^{3}\left\|\vec{\kappa}_{k}\right\|_{8,2}^{\frac{10}{16}} \max \left\{1,\left\|\vec{\kappa}_{i}\right\|_{8,2}\right\}^{\frac{3}{16}} .
\end{aligned}
$$

Finally using Lemma A.1, Corollary 3.9, and the uniform bounds on the length we obtain

$$
\left|\sum_{i=1}^{3}\left[\left\langle\nabla_{s} \nabla_{t} S_{1, i}, \nabla_{s}^{2} \nabla_{t} S_{1, i}\right\rangle\right]_{0}^{1}\right| \leq \varepsilon \sum_{i=1}^{3} \int_{0}^{1}\left|\nabla_{s}^{8} \vec{\kappa}_{i}\right|^{2} d s+C_{\epsilon}\left(\delta, \lambda_{i}, f_{i, 0}, \mathcal{L}\left(f_{i}\right), \mathcal{E}_{\lambda_{i}}\left(f_{i, 0}\right)\right) .
$$

Putting all estimates together we finally obtain

$$
\begin{aligned}
& \frac{d}{d t} \sum_{i=1}^{3} \frac{1}{2} \int_{I}\left|\nabla_{t} S_{1, i}\right|^{2} d s+\sum_{i=1}^{3} \frac{1}{2} \int_{I}\left|\nabla_{t} S_{1, i}\right|^{2} d s+\sum_{i=1}^{3} \frac{1}{2} \int_{I}\left|\nabla_{s}^{8} \vec{\kappa}_{i}\right|^{2} d s \\
& \leq \varepsilon \sum_{i=1}^{3} \int_{0}^{1}\left|\nabla_{s}^{8} \vec{\kappa}_{i}\right|^{2} d s+C_{\epsilon}\left(\delta, \lambda_{i}, f_{i, 0}, \mathcal{L}\left(f_{i}\right), \mathcal{E}_{\lambda_{i}}\left(f_{i, 0}\right)\right) .
\end{aligned}
$$

Choosing $\varepsilon$ appropriately and a Gronwall Lemma give $\sup _{(0, T)} \sum_{i=1}^{3} \frac{1}{2} \int_{I}\left|\nabla_{t} S_{1, i}\right|^{2} d s \leq C$, with $C=C\left(\delta, \lambda_{j}, f_{j, 0}, \mathcal{L}\left(f_{j}\right), \varepsilon_{\lambda_{j}}\left(f_{j, 0}\right)\right)$, $j=1,2,3$. Going back to (6.12), integrating and by the bound on $\int_{I}\left|\nabla_{t} S_{1, i}\right|^{2} d s$ we find for all $t \in(0, T)$

$$
\sum_{i=1}^{3} \frac{1}{2} \int_{I}\left|\nabla_{s}^{6} \vec{\kappa}_{i}\right|^{2} d s \leq C+\sum_{i=1}^{3} \int_{I} \sum_{\substack{[[a, b]] \leq[[8,6]] \\ c \leq 4, b \text { even }}}\left|P_{b}^{a, c}\left(\vec{\kappa}_{i}\right)\right| d s
$$




$$
\begin{gathered}
+\left|\varphi_{i}(0)\right|^{2} \sum_{i=1}^{3} \int_{I} \sum_{\substack{[[a, b]] \leq[[6,2]] \\
c \leq 3, b \text { even }}}\left|P_{b}^{a, c}\left(\vec{\kappa}_{i}\right)\right| d s \\
+\left|\varphi_{i}(0)\right|^{4} \sum_{i=1}^{3} \int_{I} \sum_{\substack{[[a, b]] \leq[[0,2]] \\
c \leq 0, b \text { even }}}\left|P_{b}^{a, c}\left(\vec{\kappa}_{i}\right)\right| d s .
\end{gathered}
$$

By (5.13) with $\ell=4$ we find

$$
\left|\varphi_{i}(0)\right| \leq \frac{C\left(\mathcal{L}\left(f_{i}\right)\right)}{\delta} \sum_{i=1}^{3}\left\|\vec{\kappa}_{i}\right\|_{6,2}^{\frac{5}{12}}
$$

and by Lemma 3.11 with $\ell=4$

$$
\begin{aligned}
& \sum_{i=1}^{3} \int_{I} \sum_{\substack{[[a, b]] \leq[[8,6]] \\
c \leq 4, \text { b even }}}\left|P_{b}^{a, c}\left(\vec{\kappa}_{i}\right)\right| d s \leq \sum_{i=1}^{3} C\left(\mathcal{L}\left(f_{i}\right)\right) \max \left\{1,\left\|\vec{\kappa}_{i}\right\|_{6,2}\right\}^{\frac{5}{3}} \\
& \sum_{i=1}^{3} \int_{I} \sum_{\substack{[[a, b]] \leq[[6,2]] \\
c \leq 3, \text { beven }}}\left|P_{b}^{a, c}\left(\vec{\kappa}_{i}\right)\right| d s \leq \sum_{i=1}^{3} C\left(\mathcal{L}\left(f_{i}\right)\right) \max \left\{1,\left\|\vec{\kappa}_{i}\right\|_{6,2}\right\} \\
& \sum_{i=1}^{3} \int_{I} \sum_{\substack{[[a, b]] \leq[[0,2]] \\
c \leq 0, \text { beven }}}\left|P_{b}^{a, c}\left(\vec{\kappa}_{i}\right)\right| d s \leq \sum_{i=1}^{3} C\left(\mathcal{L}\left(f_{i}\right)\right) .
\end{aligned}
$$

All this together with the uniform bound on the lengths, Lemma A.1 and Corollary 3.9 yields

$$
\sup _{(0, T)} \sum_{i=1}^{3} \int_{I}\left|\nabla_{s}^{6} \vec{\kappa}_{i}\right|^{2} d s \leq C
$$

where $C=C\left(\delta, \lambda_{j}, f_{j, 0}, \mathcal{L}\left(f_{j}\right), \mathcal{E}_{\lambda_{j}}\left(f_{j, 0}\right)\right), j=1,2,3$. For the tangential component we derive

$$
\sup _{(0, T)} \sum_{i=1}^{3}\left|\varphi_{i}(t, 0)\right|+\left|\partial_{s} \varphi_{i}(t, 0)\right| \leq C \quad \text { and } \quad \sup _{(0, T)} \sum_{i=1}^{3}\left(\left\|\varphi_{i}\right\|_{L^{\infty}}+\left\|\partial_{s} \varphi_{i}\right\|_{L^{\infty}}\right) \leq C
$$

by (5.13) with $\ell=4$, (6.11) and (6.7). Again here $C$ depends on $\delta, \lambda_{j}, f_{j, 0}, \mathcal{L}\left(f_{j}\right)$ and $\varepsilon_{\lambda_{j}}\left(f_{j, 0}\right)$ for $j=1,2,3$.

Induction Step: In the following we denote by

$$
S_{m, i}:=\partial_{t}^{m-1} S_{1, i}=\partial_{t}^{m-1}\left(-\nabla_{s}^{2} \vec{\kappa}_{i}+\varphi_{i} \partial_{s} f_{i}\right), \quad m \in \mathbb{N} .
$$

The induction hypothesis reads (for some $m \in \mathbb{N}$ ):

$$
\begin{array}{r}
\sup _{(0, T)} \sum_{i=1}^{3} \int_{I}\left|\nabla_{t} S_{m, i}\right|^{2} d s \leq C, \quad \sup _{(0, T)} \sum_{i=1}^{3} \int_{I}\left|\nabla_{s}^{2+4 m} \vec{\kappa}_{i}\right|^{2} d s \leq C, \\
\text { together with } \sup _{(0, T)} \sum_{i=1}^{3}\left(\sum_{k=0}^{m-1}\left|\partial_{t}^{k} \varphi_{i}(t, 0)\right|\right) \leq C,
\end{array}
$$

where $C=C\left(\delta, \lambda_{j}, f_{j, 0}, \mathcal{L}\left(f_{j}\right), \varepsilon_{\lambda_{j}}\left(f_{j, 0}\right)\right)$ for $j=1,2,3$. Thus let us consider $S_{m+1, i}$ and derive the corresponding estimates. By Lemma 3.2 with $\vec{\phi}=\nabla_{t} S_{m+1, i}$ and summing over $i$ we find

$$
\frac{d}{d t} \sum_{i=1}^{3} \frac{1}{2} \int_{I}\left|\nabla_{t} S_{m+1, i}\right|^{2} d s+\sum_{i=1}^{3} \int_{I}\left|\nabla_{s}^{2} \nabla_{t} S_{m+1, i}\right|^{2} d s
$$




$$
\begin{aligned}
=- & \sum_{i=1}^{3}\left[\left\langle\nabla_{t} S_{m+1, i}, \nabla_{s}^{3} \nabla_{t} S_{m+1, i}\right\rangle\right]_{0}^{1}+\sum_{i=1}^{3}\left[\left\langle\nabla_{s} \nabla_{t} S_{m+1, i}, \nabla_{s}^{2} \nabla_{t} S_{m+1, i}\right\rangle\right]_{0}^{1} \\
& +\sum_{i=1}^{3} \int_{I}\left\langle\left(\nabla_{t}+\nabla_{s}^{4}\right) \nabla_{t} S_{m+1, i}+\frac{1}{2} \nabla_{t} S_{m+1, i} \partial_{s} \varphi_{i}, \nabla_{t} S_{m+1, i}\right\rangle d s \\
& -\frac{1}{2} \sum_{i=1}^{3} \int_{I}\left|\nabla_{t} S_{m+1, i}\right|^{2}\left\langle\vec{\kappa}_{i}, \vec{V}_{i}\right\rangle d s .
\end{aligned}
$$

We need to compute these terms. For $m \geq 1$ we have that by calculations similar the ones performed in Remark 5.2 (that is inductively using (3.6), (3.7), Lemma 3.5, Lemma 3.6)

$$
\begin{aligned}
& S_{m+1, i}=\partial_{t}^{m}\left(-\nabla_{s}^{2} \vec{\kappa}_{i}+\varphi_{i} \partial_{s} f_{i}\right) \\
& =(-1)^{m+1} \nabla_{s}^{4 m+2} \vec{\kappa}_{i}+\sum_{\substack{[[a, b]] \leq[[4 m, 3]] \\
c \leq 4 m \\
b \text { odd }}} P_{b}^{a, c}\left(\vec{\kappa}_{i}\right)
\end{aligned}
$$

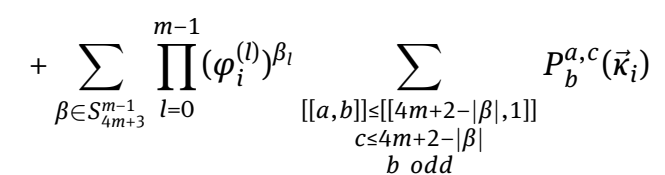

$$
\begin{aligned}
& +\partial_{s} f_{i}\left(\partial_{t}^{m} \varphi_{i}(t, x)+\sum_{\substack{[[a, b]] \leq[[4 m+1,2]] \\
c \leq 4 m-1 \\
b \text { even }}} P_{b}^{a, c}\left(\vec{\kappa}_{i}\right)\right. \\
& \left.+\sum_{\beta \in S_{4 m}^{m-1}} \prod_{l=0}^{m-1}\left(\varphi_{i}^{(l)}\right)^{\beta_{l}} \sum_{\substack{[[a, b]] \leq[[4 m+1-|\beta|, 2]] \\
c \leq 4 m+1-|\beta| \\
b \text { even }}} P_{b}^{a, c}\left(\vec{\kappa}_{i}\right)\right) .
\end{aligned}
$$

Then by Lemma 3.5, (3.16), (3.2), (3.3), and (3.6) we infer

$$
\begin{aligned}
& \nabla_{t} S_{m+1, i}=(-1)^{m+2} \nabla_{S}^{4(m+1)+2} \vec{\kappa}_{i}
\end{aligned}
$$

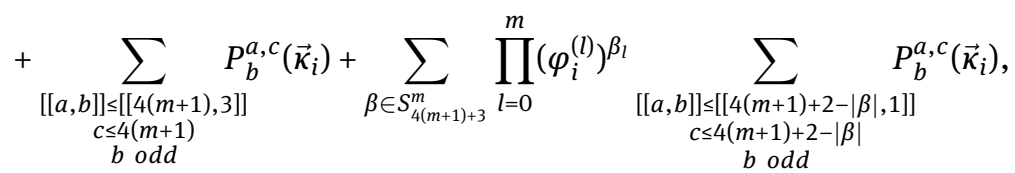

$$
\begin{aligned}
& \nabla_{t}^{2} S_{m+1, i}=(-1)^{m+1} \nabla_{s}^{4(m+2)+2} \vec{\kappa}_{i}
\end{aligned}
$$

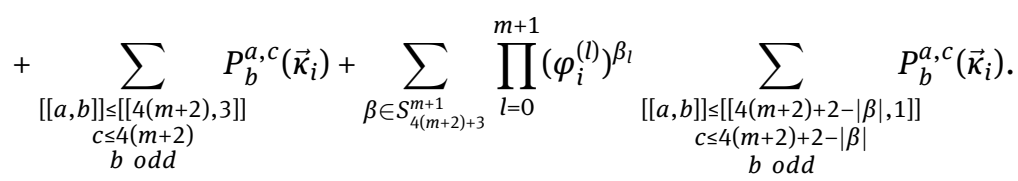

Furthermore using (3.1) we deduce

$$
\begin{aligned}
& \nabla_{s}^{2} \nabla_{t} S_{m+1, i}=(-1)^{m} \nabla_{s}^{4 m+8} \vec{\kappa}_{i}
\end{aligned}
$$

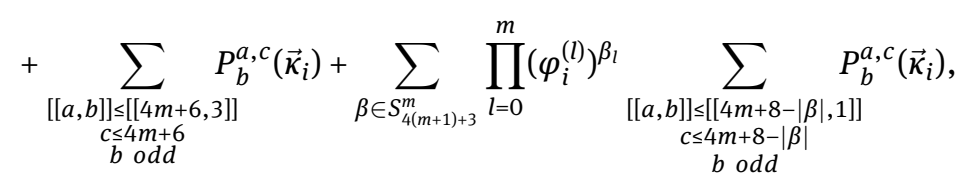

$$
\begin{aligned}
& +\sum_{\beta \in S_{4(m+1)+3}^{m}} \partial_{s}\left(\prod_{l=0}^{m}\left(\varphi_{i}^{(l)}\right)^{\beta_{l}}\right) \sum_{\substack{[[a, b]] \leq[[4(m+1)+3-|\beta|, 1]] \\
c \leq 4(m+1)+3-|\beta| \\
b \text { odd }}} P_{b}^{a, c}\left(\vec{\kappa}_{i}\right)
\end{aligned}
$$




$$
\sum_{\beta \in S_{4(m+1)+3}^{m}} \partial_{S}^{2}\left(\prod_{l=0}^{m}\left(\varphi_{i}^{(l)}\right)^{\beta_{l}}\right) \sum_{\substack{[[a, b]] \leq[[4(m+1)+2-|\beta|, 1]] \\ c \leq 4(m+1)+2-|\beta| \\ b \text { odd }}} P_{b}^{a, c}\left(\vec{\kappa}_{i}\right) .
$$

Using Lemma A.3, where the behaviour of the derivatives with respect to $s$ of time derivatives of $\varphi_{i}$ is investigated, (and the inequality $|a+b|^{2} \geq \frac{1}{2}|a|^{2}-C|b|^{2}$ ) we obtain

$$
\begin{aligned}
& \left|\nabla_{s}^{2} \nabla_{t} S_{m+1, i}\right|^{2} \geq \frac{1}{2}\left|\nabla_{s}^{4 m+8} \vec{\kappa}_{i}\right|^{2}-\sum_{\substack{[[a, b]] \leq[[8 m+12,6]] \\
c \leq 4 m+6, b \text { even }}}\left|P_{b}^{a, c}\left(\vec{\kappa}_{i}\right)\right| \\
& -\sum_{\beta \in S_{4(m+1)+3}^{m}} \prod_{\substack{l=0 \\
m}}^{m}\left(\left|\partial_{t}^{l} \varphi_{i}(t, 0)\right|+1\right)^{2 \beta_{l}} \sum_{\substack{[[a, b]] \leq[[8 m+16-2|\beta|, 2]] \\
c \leq 4 m+8-|\beta| \\
b \text { even }}}\left|P_{b}^{a, c}\left(\vec{\kappa}_{i}\right)\right| \\
& -\sum_{\beta \in S_{4(m+1)+3}^{m}} \prod_{\substack{l=0 \\
m}}^{m}\left(\left|\partial_{t}^{l} \varphi_{i}(t, 0)\right|+1\right)^{2 \beta_{l}} \sum_{\substack{[[a, b]] \leq[[8(m+1)+6-2|\beta|, 2]] \\
c \leq 4(m+1)+3-|\beta| \\
b \text { even }}}\left|P_{b}^{a, c}\left(\vec{\kappa}_{i}\right)\right|
\end{aligned}
$$

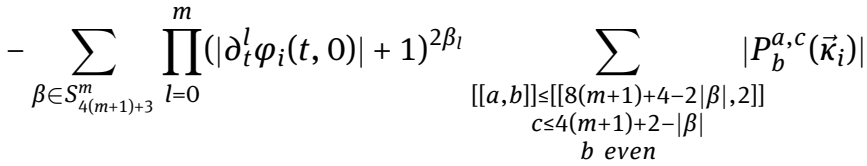

$$
\begin{aligned}
& \geq \frac{1}{2}\left|\nabla_{s}^{4 m+8} \vec{\kappa}_{i}\right|^{2}-\sum_{\substack{[[a, b]] \leq[[8 m+12,6]] \\
c \leq 4 m+6, b \text { even }}}\left|P_{b}^{a, c}\left(\vec{\kappa}_{i}\right)\right|
\end{aligned}
$$

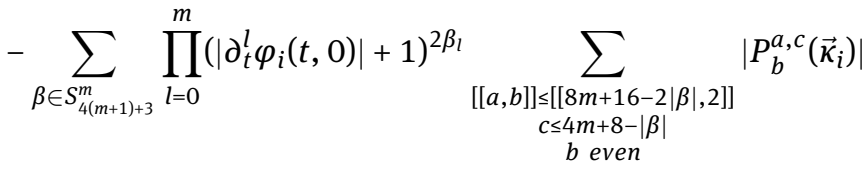

$$
\begin{aligned}
& \geq \frac{1}{2}\left|\nabla_{s}^{4 m+8} \vec{\kappa}_{i}\right|^{2}-\sum_{\substack{[[a, b]] \leq[[8 m+12,6]] \\
c \leq 4 m+6, b \text { even }}}\left|P_{b}^{a, c}\left(\vec{\kappa}_{i}\right)\right| \\
& -\left|\partial_{t}^{m} \varphi_{i}(t, 0)\right|^{2} \sum_{\substack{[[a, b]] \leq[[10,2]] \\
c \leq 5 \\
b \text { even }}}\left|P_{b}^{a, c}\left(\vec{\kappa}_{i}\right)\right|,
\end{aligned}
$$

where we have used the induction hypothethis (6.14) in the last step. Similarly

$$
\begin{aligned}
& \left|\nabla_{t} S_{m+1, i}\right|^{2} \geq \frac{1}{2}\left|\nabla_{s}^{4(m+1)+2} \vec{\kappa}_{i}\right|^{2}
\end{aligned}
$$

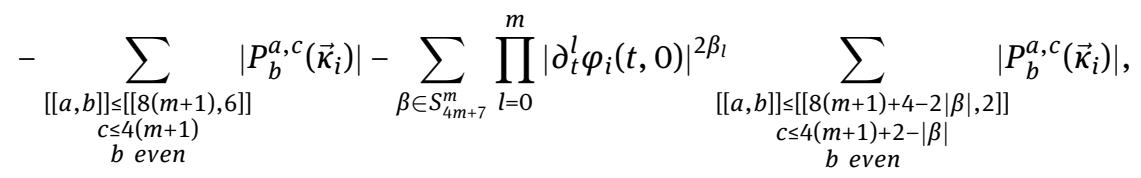

$$
\begin{aligned}
& \geq \frac{1}{2}\left|\nabla_{s}^{4(m+1)+2} \vec{\kappa}_{i}\right|^{2} \\
& -\sum_{\substack{[[a, b]] \leq[[8(m+1), 6]] \\
c \leq 4(m+1) \\
b \text { even }}}\left|P_{b}^{a, c}\left(\vec{\kappa}_{i}\right)\right|-\left|\partial_{t}^{m} \varphi_{i}(t, 0)\right|^{2} \sum_{\substack{[[a, b]] \leq[[6,2]] \\
c \leq 3 \\
b \text { even }}}\left|P_{b}^{a, c}\left(\vec{\kappa}_{i}\right)\right|,
\end{aligned}
$$

and again with Lemma A.3

$$
\left|\left(\nabla_{t}+\nabla_{s}^{4}\right) \nabla_{t} S_{m+1, i}\right| \leq \sum_{\substack{[[a, b]] \leq[[4 m+8,3]] \\ c \leq 4 m+8 \\ b \text { odd }}}\left|P_{b}^{a, c}\left(\vec{\kappa}_{i}\right)\right|
$$




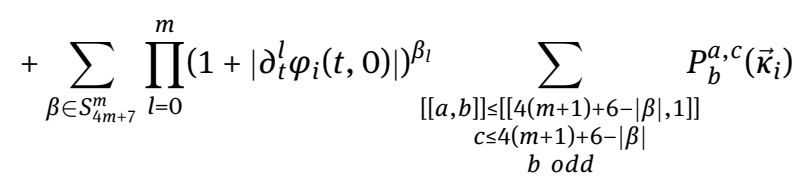

$$
\begin{aligned}
& +\sum_{\beta \in S_{4(m+2)+3}^{m+1}} \prod_{\substack{l=0 \\
c \leq 4(m+2)+2-|\beta| \\
b \text { odd }}}^{m+1}\left|\varphi_{i}^{(l)}\right|^{\beta_{l}} \sum_{\substack{[[a, b]] \leq[[4(m+2)+2-|\beta|, 1]] \\
c}}^{a, c}\left(\vec{\kappa}_{i}\right) \mid \\
& \leq \sum_{\substack{[[a, b]] \leq[[4 m+8,3]] \\
c \leq 4 m+8 \\
b \text { odd }}}\left|P_{b}^{a, c}\left(\vec{\kappa}_{i}\right)\right|+\left|\partial_{t}^{m} \varphi_{i}(t, 0)\right| \sum_{\substack{[[a, b]] \leq[[7,1]] \\
c \leq 7 \\
b \text { odd }}} P_{b}^{a, c}\left(\vec{\kappa}_{i}\right) \\
& +\left|\partial_{t}^{m+1} \varphi_{i}(t, 0)\right| \sum_{\substack{[[a, b]] \leq[[3,1]] \\
c \leq 3 \\
b \text { odd }}}\left|P_{b}^{a, c}\left(\vec{\kappa}_{i}\right)\right|+\left|\partial_{t}^{m} \varphi_{i}(t, 0)\right|^{2} \sum_{\substack{[[a, b]] \leq[[0,1]] \\
c \leq 0 \\
b \text { odd }}}\left|P_{b}^{a, c}\left(\vec{\kappa}_{i}\right)\right|
\end{aligned}
$$

where for the second inequality we have used the induction hypothethis (6.14) and where the term mutiplying $\left|\partial_{t}^{m} \varphi_{i}(t, 0)\right|^{2}$ actually appears only when $m=1$.

From (6.15) we obtain adding the term $\frac{1}{2} \int_{I}\left|\nabla_{t} S_{m+1, i}\right|^{2} d s$ and using the expressions derived above (and recalling (6.11) and the fact that $m \geq 1$ )

$$
\begin{aligned}
& \frac{d}{d t} \sum_{i=1}^{3} \frac{1}{2} \int_{I}\left|\nabla_{t} S_{m+1, i}\right|^{2} d s+\sum_{i=1}^{3} \frac{1}{2} \int_{I}\left|\nabla_{t} S_{m+1, i}\right|^{2} d s+\sum_{i=1}^{3} \frac{1}{2} \int_{I}\left|\nabla_{s}^{4 m+8} \vec{\kappa}_{i}\right|^{2} d s \\
& \leq-\sum_{i=1}^{3}\left[\left\langle\nabla_{t} S_{m+1, i}, \nabla_{s}^{3} \nabla_{t} S_{m+1, i}\right\rangle\right]_{0}^{1}+\sum_{i=1}^{3}\left[\left\langle\nabla_{s} \nabla_{t} S_{m+1, i}, \nabla_{s}^{2} \nabla_{t} S_{m+1, i}\right\rangle\right]_{0}^{1} \\
& +\sum_{i=1}^{3} \int_{I} \sum_{\substack{[[a, b]] \leq[[8 m+14,4]] \\
c \leq 4 m+8 \\
b \text { even }}}\left|P_{b}^{a, c}\left(\vec{\kappa}_{i}\right)\right| d s+\sum_{i=1}^{3}\left|\partial_{t}^{m} \varphi_{i}(t, 0)\right| \int_{I} \sum_{\substack{[[a, b]] \leq[[4 m+13,2]] \\
c \leq 4 m+8 \\
b \text { even }}}\left|P_{b}^{a, c}\left(\vec{\kappa}_{i}\right)\right| d s
\end{aligned}
$$

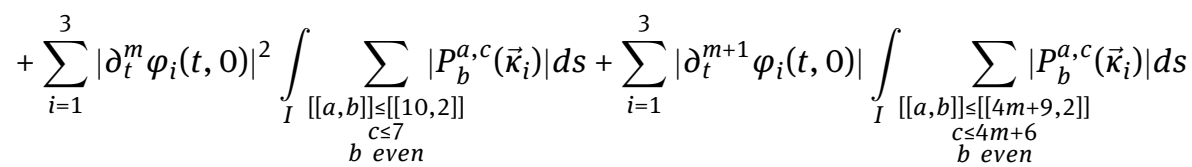

$$
\begin{aligned}
& +\sum_{i=1}^{3}\left|\partial_{t}^{m+1} \varphi_{i}(t, 0)\right|\left|\partial_{t}^{m} \varphi_{i}(t, 0)\right| \int_{\substack { I \\
\begin{subarray}{c}{[[a, b]] \leq \leq[6,2]] \\
b \leq 3 \\
b \text { even }{ I \\
\begin{subarray} { c } { [ [ a , b ] ] \leq \leq [ 6 , 2 ] ] \\
b \leq 3 \\
b \text { even } } }\end{subarray}}\left|P_{b}^{a, c}\left(\vec{\kappa}_{i}\right)\right| d s \\
& +\sum_{i=1}^{3}\left|\partial_{t}^{m} \varphi_{i}(t, 0)\right|^{2} \int_{I} \sum_{\substack{[[a, b]] \leq[[4 m+6,2]] \\
c \leq 4 m+6 \\
b \text { even }}}\left|P_{b}^{a, c}\left(\vec{\kappa}_{i}\right)\right|+\left|\partial_{t}^{m} \varphi_{i}(t, 0)\right|^{3} \int_{\substack{I[a, b]] \leq[[3,2]] \\
c \leq 3 \\
b \text { even }}}\left|P_{b}^{a, c}\left(\vec{\kappa}_{i}\right)\right| d s,
\end{aligned}
$$

where the last two integrals appear only if $m=1$. We first estimate the integral terms. By Lemma 3.11 with $\ell=4 m+6$ we obtain

$$
\sum_{i=1}^{3} \int_{I} \sum_{\substack{[[a, b]] \leq[[8 m+14,4]] \\ c \leq 4 m+8, b \text { even }}}\left|P_{b}^{a, c}\left(\vec{\kappa}_{i}\right)\right| \leq \varepsilon \sum_{i=1}^{3} \int_{0}^{1}\left|\nabla_{s}^{4 m+8} \vec{\kappa}_{i}\right|^{2} d s+C_{\epsilon}\left(\lambda_{i}, f_{i, 0}, \mathcal{L}\left(f_{i}\right), \mathcal{E}_{\lambda_{i}}\left(f_{i, 0}\right)\right),
$$

with $\varepsilon \in(0,1)$. By the same interpolation result and (5.15), again with $\ell=4 m+6$, we get

$$
\sum_{i=1}^{3}\left|\partial_{t}^{m} \varphi_{i}(t, 0)\right| \int_{I} \sum_{\substack{[[a, b]] \leq[[4 m+13,2]] \\ c \leq 4 m+8 \\ b \text { even }}}\left|P_{b}^{a, c}\left(\vec{\kappa}_{i}\right)\right| d s
$$




$$
\begin{aligned}
& +\sum_{i=1}^{3}\left|\partial_{t}^{m} \varphi_{i}(t, 0)\right|^{2} \int_{I} \sum_{\substack{[[a, b]] \leq[[10,2]] \\
c \leq 7 \\
b \text { even }}}\left|P_{b}^{a, c}\left(\vec{\kappa}_{i}\right)\right| d s \\
& +\sum_{i=1}^{3}\left|\partial_{t}^{m+1} \varphi_{i}(t, 0)\right| \int_{I} \sum_{\substack{[[a, b]] \leq[[4 m+9,2]] \\
c \leq 4 m+6 \\
b \text { even }}}\left|P_{b}^{a, c}\left(\vec{\kappa}_{i}\right)\right| d s \\
& +\sum_{i=1}^{3}\left|\partial_{t}^{m+1} \varphi_{i}(t, 0) \| \partial_{t}^{m} \varphi_{i}(t, 0)\right| \int_{\substack{I[a, b]] \leq[[6,2]] \\
c \leq 3 \\
b \text { even }}}\left|P_{b}^{a, c}\left(\vec{\kappa}_{i}\right)\right| d s \\
& \leq C(m, \delta) \sum_{i=1}^{3} \sum_{k=1}^{3}\left(\max \left\{1,\left\|\vec{\kappa}_{k}\right\|_{4 m+8,2}\right\}^{\frac{(5+8 m)}{2(4 m+8)}} \max \left\{1,\left\|\vec{\kappa}_{i}\right\|_{4 m+8,2}\right\}^{\frac{4 m+13}{4 m+8}}\right. \\
& +\max \left\{1,\left\|\vec{\kappa}_{k}\right\|_{4 m+8,2}\right\}^{\frac{2(5+8 m)}{2(4 m+8)}} \max \left\{1,\left\|\vec{\kappa}_{i}\right\|_{4 m+8,2}\right\}^{\frac{10}{4 m+8}} \\
& +\max \left\{1,\left\|\vec{\kappa}_{k}\right\|_{4 m+8,2}\right\}^{\frac{(13+8 m)}{2(4 m+8)}} \max \left\{1,\left\|\vec{\kappa}_{i}\right\|_{4 m+8,2}\right\}^{\frac{4 m+9}{4 m+8}} \\
& \left.+\sum_{l=1}^{3} \max \left\{1,\left\|\vec{\kappa}_{k}\right\|_{4 m+8,2}\right\}^{\frac{(5+8 m)}{2(4 m+8)}} \max \left\{1,\left\|\vec{\kappa}_{l}\right\|_{4 m+8,2}\right\}^{\frac{(13+8 m)}{2(4 m+8)}} \max \left\{1,\left\|\vec{\kappa}_{i}\right\|_{4 m+8,2}\right\}^{\frac{6}{4 m+8}}\right) \\
& \leq \varepsilon \sum_{i=1}^{3} \int_{0}^{1}\left|\nabla_{s}^{4 m+8} \vec{\kappa}_{i}\right|^{2} d s+C_{\epsilon}\left(\delta, \lambda_{i}, f_{i, 0}, \mathcal{L}\left(f_{i}\right), \mathcal{E}_{\lambda_{i}}\left(f_{i, 0}\right)\right) \text {, }
\end{aligned}
$$

where we have used Corollary 3.9, the uniform bound on the lengths and Lemma A.1 in the last inequality. The last two integral terms in (6.21) have to be evaluated only when $m=1$. In this case we calculate with the same arguments as above and (6.13)

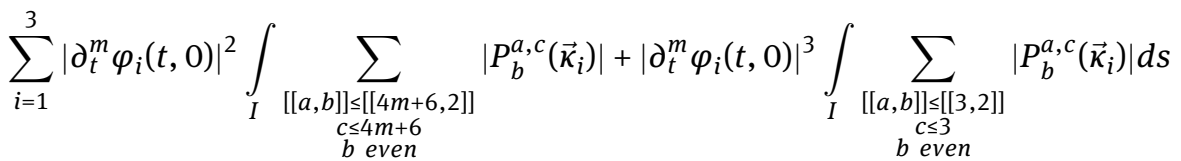

$$
\begin{aligned}
& \leq C(m, \delta) \sum_{i=1}^{3} \sum_{k=1}^{3} \max \left\{1,\left\|\vec{\kappa}_{k}\right\|_{4 m+8,2}\right\}^{\frac{2(5+8 m)}{2(4 m+8)}} \max \left\{1,\left\|\vec{\kappa}_{i}\right\|_{4 m+8,2}\right\}^{\frac{4 m+6}{4 m+8}} \\
& +C(m, \delta) \sum_{i=1}^{3} \sum_{k=1}^{3} \max \left\{1,\left\|\vec{\kappa}_{k}\right\|_{4 m+8,2}\right\}^{\frac{3(5+8 m)}{2(4 m+8)}} \max \left\{1,\left\|\vec{\kappa}_{i}\right\|_{4 m+2,2}\right\}^{\frac{3}{4 m+2}} \\
& \leq C(m, \delta) \sum_{i=1}^{3} \sum_{k=1}^{3} \max \left\{1,\left\|\vec{\kappa}_{k}\right\|_{4 m+8,2}\right\}^{\frac{2(5+8 m)}{2(4 m+8)}} \max \left\{1,\left\|\vec{\kappa}_{i}\right\|_{4 m+8,2}\right\}^{\frac{4 m+6}{4 m+8}} \\
& +C(m, \delta) \sum_{i=1}^{3} \sum_{k=1}^{3} \max \left\{1,\left\|\vec{\kappa}_{k}\right\|_{4 m+8,2}\right\}^{\frac{3(5+8 m)}{2(4 m+8)}} \\
& \leq \varepsilon \sum_{i=1}^{3} \int_{0}^{1}\left|\nabla_{s}^{4 m+8} \vec{\kappa}_{i}\right|^{2} d s+C_{\epsilon}\left(\delta, \lambda_{i}, f_{i, 0}, \mathcal{L}\left(f_{i}\right), \mathcal{E}_{\lambda_{i}}\left(f_{i, 0}\right)\right),
\end{aligned}
$$

where, we have appplied Lemma A.1 and Corollary 3.9 in the last step since by $m=1$ we have that $\frac{3(5+8 m)}{2(4 m+8)}<2$ and similarly $2(5+8 m)+2(4 m+6)<4(4 m+8)$.

It remains to treat the boundary terms. We can write

$$
-\sum_{i=1}^{3}\left[\left\langle\nabla_{t} S_{m+1, i}, \nabla_{s}^{3} \nabla_{t} S_{m+1, i}\right\rangle\right]_{0}^{1}=-\sum_{i=1}^{3}\left[\left\langle\partial_{t} S_{m+1, i}, \nabla_{s}^{3} \nabla_{t} S_{m+1, i}\right\rangle\right]_{0}^{1}
$$


Since $\partial_{t} S_{m+1, i}=\partial_{t}^{m+1} f_{i}$ at the boundary points, and here $\partial_{t}^{m+1} f_{i}=\partial_{t}^{m+1} f_{j}$, for $i, j=1,2,3$, we find

$$
-\sum_{i=1}^{3}\left[\left\langle\nabla_{t} S_{m+1, i}, \nabla_{S}^{3} \nabla_{t} S_{m+1, i}\right\rangle\right]_{0}^{1}=\left.\left\langle\partial_{t} S_{m+1,1}, \sum_{i=1}^{3} \nabla_{S}^{3} \nabla_{t} S_{m+1, i}\right\rangle\right|_{x=0} .
$$

Since from (6.19)

$$
\begin{aligned}
& \nabla_{s}^{3}\left(\nabla_{t} S_{m+1, i}\right)=(-1)^{m} \nabla_{s}^{4(m+1)+5} \vec{\kappa}_{i} \\
& +\sum_{\substack{[[a, b]] \leq[[4(m+1)+3,3]] \\
c \leq 4(m+1)+3 \\
b \text { odd }}} P_{b}^{a, c}\left(\vec{\kappa}_{i}\right)+\nabla_{s}^{3}\left(\sum_{\beta \in S_{4(m+1)+3}^{m}} \prod_{\substack { l=0 \\
\begin{subarray}{c}{[[a, b]] \leq[4(m+1)+2-|\beta|, 1]] \\
c \leq 4(m+1)+2-|\beta| \\
b \text { odd }{ l = 0 \\
\begin{subarray} { c } { [ [ a , b ] ] \leq [ 4 ( m + 1 ) + 2 - | \beta | , 1 ] ] \\
c \leq 4 ( m + 1 ) + 2 - | \beta | \\
b \text { odd } } }\end{subarray}}^{m}\left(\varphi_{i}^{(l)}\right)^{\beta_{l}} P_{b}^{a, c}\left(\vec{\kappa}_{i}\right)\right),
\end{aligned}
$$

we get using (4.7)

$$
\begin{aligned}
& \sum_{i=1}^{3} \nabla_{S}^{3}\left(\nabla_{t} S_{m+1, i}\right)=\sum_{i=1}^{3}\left(\sum_{\substack{[[a, b]] \leq[[5+4(m+1)-2,3]] \\
c \leq 5+4(m+1)-2 \\
b \text { odd }}} P_{b}^{a, c}\left(\vec{\kappa}_{i}\right)\right.
\end{aligned}
$$

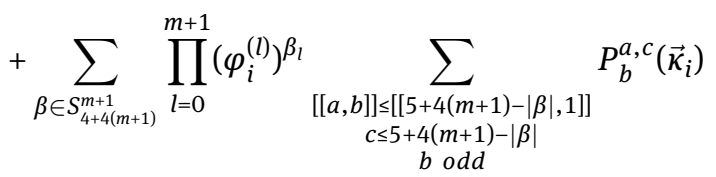

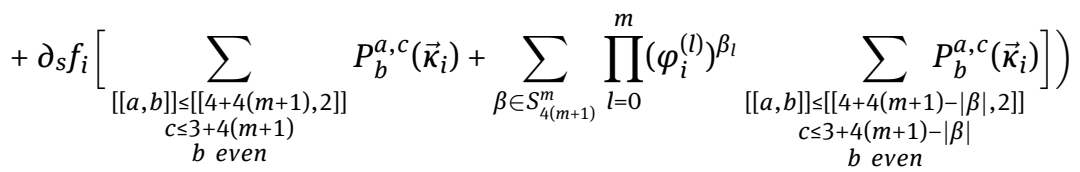

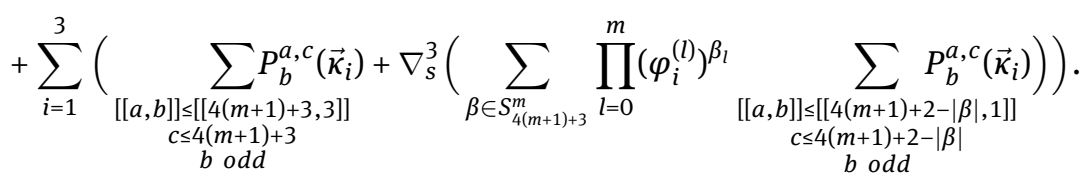

Therefore we infer using (6.14) and Lemma A.3

$$
\begin{aligned}
& \left|\sum_{i=1}^{3} \nabla_{S}^{3}\left(\nabla_{t} S_{m+1, i}\right)\right|_{x=0} \leq \sum_{i=1}^{3}\left(\left|\sum_{\substack{[[a, b]] \leq[[4(m+1)+3,3]] \\
c \leq 4(m+1)+3 \\
b \text { odd }}} P_{b}^{a, c}\left(\vec{\kappa}_{i}\right)\right|\right. \\
& +\left|\partial_{t}^{m+1} \varphi_{i}(t, 0)\right| \sum_{\substack{[[a, b]] \leq[[2,1]] \\
c \leq 2 \\
b \text { odd }}}\left|P_{b}^{a, c}\left(\vec{\kappa}_{i}\right)\right|+\left|\partial_{t}^{m} \varphi_{i}(t, 0)\right| \sum_{\substack{[[a, b]] \leq[[6,1]] \\
b \leq \leq 6 \\
b \text { odd }}}\left|P_{b}^{a, c}\left(\vec{\kappa}_{i}\right)\right| \\
& \left.+\sum_{\substack{[[a, b]] \leq[[4+4(m+1), 2]] \\
c \leq 3+4(m+1) \\
b \text { even }}}\left|P_{b}^{a, c}\left(\vec{\kappa}_{i}\right)\right|+\left|\partial_{t}^{m} \varphi_{i}(t, 0)\right| \sum_{\begin{array}{c}
{[[a, b]] \leq[[5,2]]} \\
c \leq 5 \\
b \text { even }
\end{array}}\left|P_{b}^{a, c}\left(\vec{\kappa}_{i}\right)\right|\right) .
\end{aligned}
$$

We write

$$
\sum_{\substack{[[a, b]] \leq[[4(m+1)+3,3]] \\ c \leq 4(m+1)+3 \\ b \text { odd }}} P_{b}^{a, c}\left(\vec{\kappa}_{i}\right) \sum_{\substack{[[a, b]] \leq[[4(m+1)+3,3]] \\ c \leq 4(m+1)+3 \\ b \text { odd } b \neq 1}} P_{b}^{a, c}\left(\vec{\kappa}_{i}\right)+\sum_{\substack{[[a, b]] \leq[[4(m+1)+3,3]] \\ c \leq 4(m+1)+3 \\ b=1}} P_{b}^{a, c}\left(\vec{\kappa}_{i}\right)
$$

and estimate by (3.23)

$$
\sum_{\substack{[[a, b]] \leq[[4(m+1)+3,3]] \\ c \leq 4(m+1)+3 \\ b \text { odd }, b \neq 1}} P_{b}^{a, c}\left(\vec{\kappa}_{i}\right) \leq \int_{I} \sum_{\substack{[[a, b]] \leq[[4(m+1)+4,3]] \\ c \leq 4(m+1)+4 \\ b \geq 2}}\left|P_{b}^{a, c}\left(\vec{\kappa}_{i}\right)\right| d s .
$$


Next, using Lemma 3.7, Lemma 3.11 with $\ell=4 m+6$, and (5.15) we derive

$$
\begin{aligned}
& \left|\sum_{i=1}^{3} \nabla_{s}^{3}\left(\nabla_{t} S_{m+1, i}\right)\right|_{x=0} \leq \sum_{i=1}^{3}\left(\int_{I} \sum_{\substack{[[a, b]] \leq[[4(m+1)+4,3]] \\
c \leq 4(m+1)+4 \\
b \geq 2}}\left|P_{b}^{a, c}\left(\vec{\kappa}_{i}\right)\right| d s\right. \\
& +\left(\int_{I} \sum_{\substack{[[a, b]] \leq[[8(m+1)+7,2]] \\
c \leq 4(m+1)+4 \\
b \text { even }}}\left|P_{b}^{a, c}\left(\vec{\kappa}_{i}\right)\right| d s\right)^{\frac{1}{2}}+\left|\partial_{t}^{m+1} \varphi_{i}(t, 0)\right|\left(\int_{I} \sum_{\substack{[[a, b]] \leq[[5,2]] \\
c \leq 3 \\
b \text { even }}}\left|P_{b}^{a, c}\left(\vec{\kappa}_{i}\right)\right| d s\right)^{\frac{1}{2}} \\
& +\left|\partial_{t}^{m} \varphi_{i}(t, 0)\right|\left(\int_{I} \sum_{\substack{[[a, b]] \leq[[13,2]] \\
c \leq 7 \\
b \text { even }}}\left|P_{b}^{a, c}\left(\vec{\kappa}_{i}\right)\right| d s\right)^{\frac{1}{2}}+\int_{I} \sum_{\substack{[[a, b]] \leq[[4(m+1)+5,2]] \\
c \leq 4(m+1)+4 \\
b \text { even }}}\left|P_{b}^{a, c}\left(\vec{\kappa}_{i}\right)\right| d s
\end{aligned}
$$

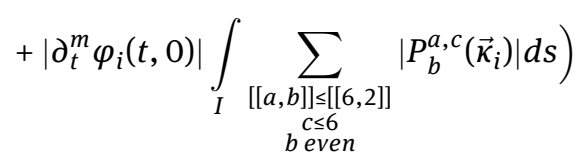

$$
\begin{aligned}
& \leq \sum_{i=1}^{3}\left(\max \left\{1,\left\|\vec{\kappa}_{i}\right\|_{4 m+8,2}\right\}^{\frac{8 m+18}{2(4 m+8)}}\right. \\
& +C(m, \delta) \sum_{k=1}^{3} \max \left\{1,\left\|\vec{\kappa}_{k}\right\|_{4 m+8,2}\right\}^{\frac{8 m+13}{2(4 m+8)}} \max \left\{1,\left\|\vec{\kappa}_{i}\right\|_{4 m+8,2}\right\}^{\frac{5}{2(4 m+8)}} \\
& \left.+C(m, \delta) \sum_{k=1}^{3} \max \left\{1,\left\|\vec{\kappa}_{k}\right\|_{4 m+8,2}\right\}^{\frac{8 m+5}{2(4 m+8)}} \max \left\{1,\left\|\vec{\kappa}_{i}\right\|_{4 m+8,2}\right\}^{\frac{13}{2(4 m+8)}}\right) .
\end{aligned}
$$

Next we observe that by (6.16) we have

$$
\begin{aligned}
\partial_{t} S_{m+1, i}= & S_{m+2, i}=\partial_{t}^{m+1}\left(-\nabla_{s}^{2} \vec{\kappa}_{i}+\varphi_{i} \partial_{s} f_{i}\right) \\
= & (-1)^{m} \nabla_{s}^{4(m+1)+2} \vec{\kappa}_{i}+\sum_{\substack{[[a, b]] \leq[[4(m+1), 3]] \\
c \leq 4(m+1) \\
b \text { odd }}} P_{b}^{a, c}\left(\vec{\kappa}_{i}\right) \\
& +\sum_{\beta \in S_{4(m+1)+3}^{m}} \prod_{l=0}^{m}\left(\varphi_{i}^{(l)}\right)^{\beta_{l}} \sum_{\substack{[[a, b]] \leq[[4(m+1)+2-|\beta|, 1]] \\
c \leq 4(m+1)+2-|\beta| \\
b \text { odd }}} P_{b}^{a, c}\left(\vec{\kappa}_{i}\right) \\
& +\partial_{s} f_{i}\left(\partial_{t}^{m+1} \varphi_{i}(t, 0)+\sum_{\substack{[[a, b]] \leq[[4(m+1)+1,2]] \\
c \leq 4(m+1)-1 \\
b \text { even }}} P_{b}^{a, c}\left(\vec{\kappa}_{i}\right)\right. \\
& +\sum_{\beta \in S_{4(m+1)}^{m}} \prod_{l=0}^{m}\left(\varphi_{i}^{(l)}\right)_{\substack{\beta_{l}}}^{\substack{[[a, b]] \leq[[4(m+1)+1-|\beta|, 2]] \\
c \leq 4(m+1)+1-|\beta| \\
b \text { even }}}
\end{aligned}
$$

so that by Lemma A.3, Lemma 3.7, Lemma 3.11 with $\ell=4 m+6$, and (5.15), we infer

$$
\begin{aligned}
\left|\partial_{t} S_{m+1, i}(0)\right| & =\left(\int_{I} \sum_{\substack{[[a, b]] \leq[[8 m+13,2]] \\
c \leq 4 m+7 \\
b \text { even }}}\left|P_{b}^{a, c}\left(\vec{\kappa}_{i}\right)\right| d s\right)^{\frac{1}{2}}+\left|\partial_{t}^{m} \varphi_{i}(t, 0)\right|\left(\int_{I} \sum_{\substack{[[a, b]] \leq[[7,2]] \\
c \leq 7 \\
b \text { even } \\
b}}\left|P_{b}^{a, c}\left(\vec{\kappa}_{i}\right)\right| d s\right)^{\frac{1}{2}} \\
& +\left|\partial_{t}^{m+1} \varphi_{i}(t, 0)\right|+\int_{I} \sum_{\substack{[[a, b]] \leq[[4 m+6,2]] \\
c \leq 4 m+4 \\
b \text { even }}}\left|P_{b}^{a, c}\left(\vec{\kappa}_{i}\right)\right| d s \\
& +\left|\partial_{t}^{m} \varphi_{i}(t, 0)\right| \int_{I} \sum_{\substack{[[a, b]] \leq[[3,2]] \\
c \leq 3 \\
\text { beven }}}\left|P_{b}^{a, c}\left(\vec{\kappa}_{i}\right)\right| d s
\end{aligned}
$$




$$
\begin{aligned}
& \leq \max \left\{1,\left\|\vec{\kappa}_{i}\right\|_{4 m+8,2}\right\}^{\frac{8 m+13}{2(4 m+8)}} \\
& +C(m, \delta) \sum_{k=1}^{3} \max \left\{1,\left\|\vec{\kappa}_{k}\right\|_{4 m+8,2}\right\}^{\frac{8 m+13}{2(4 m+8)}}+\max \left\{1,\left\|\vec{\kappa}_{i}\right\|_{4 m+8,2}\right\}^{\frac{8 m+12}{2(4 m+8)}} \\
& +C(m, \delta) \sum_{k=1}^{3} \max \left\{1,\left\|\vec{\kappa}_{k}\right\|_{4 m+8,2}\right\}^{\frac{8 m+5}{2(4 m+8)}} \max \left\{1,\left\|\vec{\kappa}_{i}\right\|_{4 m+8,2}\right\}^{\frac{7}{2(4 m+8)}}
\end{aligned}
$$

Putting all estimates together, using Lemma A.1 and Corollary 3.9 we obtain

$$
\left|-\sum_{i=1}^{3}\left[\left\langle\nabla_{t} S_{m+1, i}, \nabla_{s}^{3} \nabla_{t} S_{m+1, i}\right\rangle\right]_{0}^{1}\right| \leq \varepsilon \sum_{i=1}^{3} \int_{0}^{1}\left|\nabla_{s}^{4 m+8} \vec{\kappa}_{i}\right|^{2} d s+C_{\epsilon},
$$

with $C_{\epsilon}=C_{\epsilon}\left(\delta, \lambda_{i}, f_{i, 0}, \mathcal{L}\left(f_{i}\right), \varepsilon_{\lambda_{i}}\left(f_{i, 0}\right)\right)$.

It remains to evaluate the last boundary term $\sum_{i=1}^{3}\left[\left\langle\nabla_{s} \nabla_{t} S_{m+1, i}, \nabla_{s}^{2} \nabla_{t} S_{m+1, i}\right\rangle\right]_{0}^{1}$. First of all note that by (6.17), Lemma A.3, Lemma 3.7, Lemma 3.11 with $\ell=4 m+6$, and (5.15), we have for $x \in I$

$$
\begin{aligned}
& \left|\nabla_{s} \nabla_{t} S_{m+1, i}\right|=\mid(-1)^{m+2} \nabla_{s}^{4(m+1)+3} \vec{\kappa}_{i} \\
& +\sum_{\substack{[[a, b]] \leq[[4(m+1)+1,3]] \\
c \leq 4(m+1)+1 \\
b \text { odd }}} P_{b}^{a, c}\left(\vec{\kappa}_{i}\right)+\sum_{\beta \in S_{4(m+1)+3}^{m}} \partial_{s}\left(\prod_{l=0}^{m}\left(\varphi_{i}^{(l)}\right)^{\left.\beta_{l}\right)} \sum_{\substack{[[a, b]] \leq[[4(m+1)+2-|\beta|, 1]] \\
c \leq 4(m+1)+2-|\beta| \\
b \text { odd }}} P_{b}^{a, c}\left(\vec{\kappa}_{i}\right)\right. \\
& +\sum_{\beta \in S_{4(m+1)+3}^{m}} \prod_{\substack{l=0 \\
c \leq 4(m+1)+3-|\beta| \\
b \text { odd }}}^{m}\left(\varphi_{i}^{(l)}\right)^{\beta_{l}} \sum_{\substack{[[a, b] \leq[j] \\
c, c}}^{\left.a, \vec{\kappa}_{i}\right) \mid} \\
& \leq \sum_{\substack{[[a, b]] \leq[[4(m+1)+3,1]] \\
c \leq 4(m+1)+3 \\
b \text { odd }}}\left|P_{b}^{a, c}\left(\vec{\kappa}_{i}\right)\right|+\sum_{\beta \in S_{4(m+1)+3}^{m}} \prod_{\substack{l=0 \\
l[a, b]] \leq[[4(m+1)+3-|\beta|, 1]] \\
c \leq 4(m+1)+3-|\beta| \\
b \text { odd }}}^{m}\left|\partial_{t}^{l} \varphi_{i}(t, 0)\right|^{\beta_{l}} \sum_{b}^{a, c}\left(\vec{\kappa}_{i}\right) \mid \\
& \leq\left(\int_{\substack{I[a, b]] \leq[[8(m+1)+7,2]] \\
c \leq 4(m+1)+4 \\
b \text { even }}}\left|P_{b}^{a, c}\left(\vec{\kappa}_{i}\right)\right| d s\right)^{\frac{1}{2}}+\left|\partial_{t}^{m} \varphi_{i}(t, 0)\right|\left(\int_{ \leq ( \int _ { \substack { I [ a , b ] ] \leq [ [ 8 ( m + 1 ) + 7 , 2 ] ] \\
c \leq 4 ( m + 1 ) + 4 \\
b \text { even } } } | P _ { b } ^ { a , c } ( \vec { \kappa } _ { i } ) | d s ) ^ { \frac { 1 } { 2 } } + | \partial _ { t } ^ { m } \varphi _ { i } ( t , 0 ) | ( \int _ {\left.\substack{ \substack{ [ \\
\begin{subarray}{c}{[a, b]] \leq[j, 2]] \\
c \leq 5 \\
b \text { even }} }}\end{subarray}}\left|P_{b}^{a, c}\left(\vec{\kappa}_{i}\right)\right| d s\right)^{\frac{1}{2}}}\right. \\
& \leq \max \left\{1,\left\|\vec{\kappa}_{i}\right\|_{4 m+8,2}\right\}^{\frac{8 m+15}{2(4 m+8)}} \\
& +C(m, \delta) \sum_{k=1}^{3} \max \left\{1,\left\|\vec{\kappa}_{k}\right\|_{4 m+8,2}\right\}^{\frac{8 m+5}{2(4 m+8)}} \max \left\{1,\left\|\vec{\kappa}_{i}\right\|_{4 m+8,2}\right\}^{\frac{9}{2(4 m+8)}}
\end{aligned}
$$

Next, using (6.18), (4.3) (to lower the order of the term $\left.\nabla_{s}^{4(m+2)} \vec{\kappa}_{i}\right)$, Lemma A.3, Lemma 3.7, Lemma 3.11 with $\ell=4 m+6$, and (5.15), we infer that at the boundary (thus for $x \in\{0,1\}$ ) we have

$$
\begin{aligned}
& \left|\nabla_{s}^{2} \nabla_{t} S_{m+1, i}\right|=\mid \sum_{\substack{[[a, b]] \leq[[4 m+6,3]] \\
c \leq 4 m+6 \\
b \text { odd }}} P_{b}^{a, c}\left(\vec{\kappa}_{i}\right)+\sum_{\beta \in S_{4(m+2)}^{m+1}} \prod_{\substack{l=0 \\
c \leq 1}}^{m+1}\left(\varphi_{i}^{(l)}\right)^{\beta_{l}} \sum_{\substack{[[a, b]] \leq[[4 m+8-|\beta|, 1]] \\
b \text { odd }}} P_{b}^{a, c}\left(\vec{\kappa}_{i}\right) \\
& +\sum_{\beta \in S_{4(m+1)+3}^{m}} \prod_{\substack{l=0 \\
c \leq 4 m+8-|\beta| \\
b \text { odd }}}^{m}\left(\varphi_{i}^{(l)}\right)^{\beta_{l}} \sum_{\substack{[[a, b]] \leq[[4 m+8-|\beta|, 1]] \\
c \leq 4}}^{a, c}\left(\vec{\kappa}_{i}\right) \\
& +\sum_{\beta \in S_{4(m+1)+3}^{m}} \partial_{S}\left(\prod_{l=0}^{m}\left(\varphi_{i}^{(l)}\right)^{\beta_{l}}\right) \sum_{\substack{[[a, b]] \leq[[4(m+1)+3-|\beta|, 1]] \\
c \leq 4(m+1)+3-|\beta| \\
b \text { odd }}} P_{b}^{a, c}\left(\vec{\kappa}_{i}\right)
\end{aligned}
$$




$$
\begin{aligned}
& +\sum_{\beta \in S_{4(m+1)+3}^{m}} \partial_{s}^{2}\left(\prod_{l=0}^{m}\left(\varphi_{i}^{(l)}\right)^{\beta_{l}}\right) \sum_{\substack{[[a, b]] \leq[[4(m+1)+2-|\beta|, 1]] \\
c \leq 4(m+1)+2-|\beta| \\
b \text { odd }}} P_{b}^{a, c}\left(\vec{\kappa}_{i}\right) \mid
\end{aligned}
$$

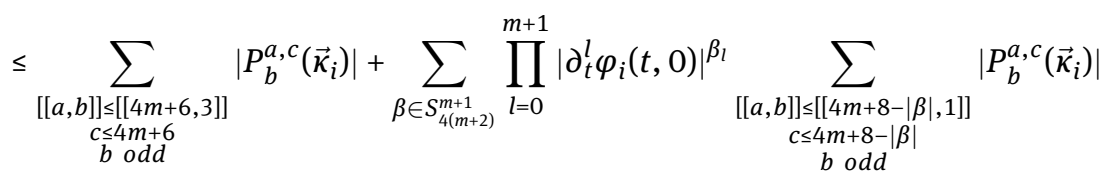

$$
\begin{aligned}
& \leq \sum_{\substack{[[a, b]] \leq[[4 m+6,3]] \\
c \leq 4 m+6 \\
b \text { odd }}}\left|P_{b}^{a, c}\left(\vec{\kappa}_{i}\right)\right|+\left|\partial_{t}^{m} \varphi_{i}(t, 0)\right| \sum_{\substack{[[a, b]] \leq[[5,1]] \\
c \leq 5 \\
b \text { odd }}}\left|P_{b}^{a, c}\left(\vec{\kappa}_{i}\right)\right| \\
& +\left|\partial_{t}^{m+1} \varphi_{i}(t, 0)\right| \sum_{\substack{[a, b]] \leq[[1,1]] \\
c \leq 1 \\
b \text { odd }}}\left|P_{b}^{a, c}\left(\vec{\kappa}_{i}\right)\right| \\
& \leq\left(\int_{I} \sum_{\substack{[[a, b]] \leq \leq[8 m+13,6]] \\
c \leq 4 m+7 \\
b \text { even }}}\left|P_{b}^{a, c}\left(\vec{\kappa}_{i}\right)\right| d s\right)^{\frac{1}{2}}+\left|\partial_{t}^{m} \varphi_{i}(t, 0)\right|\left(\int_{\substack{[[a, b]] \leq[[11,2]] \\
c \leq 6 \\
b \text { even }}}\left|P_{b}^{a, c}\left(\vec{\kappa}_{i}\right)\right| d s\right)^{\frac{1}{2}} \\
& +\left|\partial_{t}^{m+1} \varphi_{i}(t, 0)\right|\left(\int_{I} \sum_{\substack{[[a, b]] \leq[[3,2]] \\
c \leq 2 \\
b \text { even }}}\left|P_{b}^{a, c}\left(\vec{\kappa}_{i}\right)\right| d s\right)^{1 / 2} \\
& \leq \max \left\{1,\left\|\vec{\kappa}_{i}\right\|_{4 m+8,2}\right\}^{\frac{8 m+15}{2(4 m+8)}} \\
& +C(m, \delta) \sum_{k=1}^{3} \max \left\{1,\left\|\vec{\kappa}_{k}\right\|_{4 m+8,2}\right\}^{\frac{8 m+5}{2(4 m+8)}} \max \left\{1,\left\|\vec{\kappa}_{i}\right\|_{4 m+8,2}\right\}^{\frac{11}{2(4 m+8)}} \\
& +C(m, \delta) \sum_{k=1}^{3} \max \left\{1,\left\|\vec{\kappa}_{k}\right\|_{4 m+8,2}\right\}^{\frac{8 m+13}{2(4 m+8)}} \max \left\{1,\left\|\vec{\kappa}_{i}\right\|_{4 m+8,2}\right\}^{\frac{3}{2(4 m+8)}} .
\end{aligned}
$$

Putting the estimates together using Lemma A.1 and Corollary 3.9 we obtain

$$
\left|\sum_{i=1}^{3}\left[\left\langle\nabla_{s} \nabla_{t} S_{m+1, i}, \nabla_{s}^{2} \nabla_{t} S_{m+1, i}\right\rangle\right]_{0}^{1}\right| \leq \varepsilon \sum_{i=1}^{3} \int_{0}^{1}\left|\nabla_{s}^{4 m+8} \vec{\kappa}_{i}\right|^{2} d s+C_{\epsilon},
$$

with $C_{\epsilon}=C_{\epsilon}\left(\delta, \lambda_{i}, f_{i, 0}, \mathcal{L}\left(f_{i}\right), \varepsilon_{\lambda_{i}}\left(f_{i, 0}\right)\right.$. Putting the estimates together, obtained for the boundary terms and the integral terms in (6.21), we can finally state

$$
\begin{aligned}
& \frac{d}{d t} \sum_{i=1}^{3} \frac{1}{2} \int_{I}\left|\nabla_{t} S_{m+1, i}\right|^{2} d s+\sum_{i=1}^{3} \frac{1}{2} \int_{I}\left|\nabla_{t} S_{m+1, i}\right|^{2} d s+\sum_{i=1}^{3} \frac{1}{2} \int_{I}\left|\nabla_{s}^{4 m+8} \vec{\kappa}_{i}\right|^{2} d s \\
& \leq \varepsilon \sum_{i=1}^{3} \int_{0}^{1}\left|\nabla_{s}^{4 m+8} \vec{\kappa}_{i}\right|^{2} d s+C_{\epsilon}\left(\delta, \lambda_{i}, f_{i, 0}, \mathcal{L}\left(f_{i}\right), \mathcal{E}_{\lambda_{i}}\left(f_{i, 0}\right)\right) .
\end{aligned}
$$

Choosing $\varepsilon$ appropriately and applying Gronwall Lemma give that

$$
\sup _{(0, T)} \sum_{i=1}^{3} \int_{I}\left|\nabla_{t} S_{m+1, i}\right|^{2} d s \leq C=C\left(\delta, \lambda_{j}, f_{j, 0}, \mathcal{L}\left(f_{j}\right), \mathcal{E}_{\lambda_{j}}\left(f_{j, 0}\right)\right) \text { for } j=1,2,3 .
$$

Together with (6.20), Lemma 3.11 and (5.15) with $\ell=4 m+4$, Corollary 3.9, the uniform bound on the lengths and Young inequality, standard arguments yield

$$
\sup _{(0, T)} \sum_{i=1}^{3} \int_{I}\left|\nabla_{s}^{4 m+6} \vec{\kappa}_{i}\right|^{2} d s \leq C\left(\delta, \lambda_{j}, f_{j, 0}, \mathcal{L}\left(f_{j}\right), \mathcal{E}_{\lambda_{j}}\left(f_{j, 0}\right)\right) \text { for } j=1,2,3,
$$


and also

$$
\sup _{(0, T)} \sum_{i=1}^{3}\left\|\partial_{t}^{m} \varphi_{i}(t, \cdot)\right\|_{L^{\infty}(I)} \leq C\left(\delta, \lambda_{j}, f_{j, 0}, \mathcal{L}\left(f_{j}\right), \varepsilon_{\lambda_{j}}\left(f_{j, 0}\right)\right) \text { for } j=1,2,3,
$$

by (5.15) with $\ell=4 m+4$, and Lemma A.3.

Fourth Step: Smooth extension up to $T$

First of all we show that for all $t \in(0, T)$

$$
\sum_{i=1}^{3}\left\|\partial_{x}^{m} \vec{\kappa}_{i}\right\|_{C^{0}(\bar{I})} \leq C=C\left(m, \delta, n, T, \mathcal{L}\left(f_{j}\right), \mathcal{E}_{\lambda}\left(f_{j, 0}\right), f_{j, 0}, \lambda_{j}\right) \text { with } j=1,2,3 .
$$

From the previous step, Lemma 3.4, embedding inequalities (see for instance the first inequality in (3.23)) and the fact that the length of the curves remains uniformly bounded along the flow we can state that

$$
\sum_{i=1}^{3}\left\|\partial_{s}^{m} \vec{\kappa}_{i}\right\|_{C^{0}(\bar{I})} \leq C\left(m, \delta, n, \mathcal{L}\left(f_{j}\right), \mathcal{E}_{\lambda}\left(f_{j, 0}\right), f_{j, 0}, \lambda_{j}\right) \text { with } j=1,2,3,
$$

for any $m \in \mathbb{N}$ and $t \in(0, T)$. From now on the proof follows most of the arguments depicted in [8, $\S 5$ (Step seventh onwards)] with just minimal changes due to the presence of the tangential component that we point out. For the sake of completeness we sketch here again the main ideas. In the following let $\gamma_{i}:=\left|\partial_{x} f_{i}\right|$. By induction it can be proven that for any function $h: \bar{I} \rightarrow \mathbb{R}$ or vector field $h: \bar{I} \rightarrow \mathbb{R}^{n}$, and for any $m \in \mathbb{N}$

$$
\partial_{x}^{m} h=\gamma^{m} \partial_{s}^{m} h+\sum_{j=1}^{m-1} P_{m-1}\left(\gamma, . ., \partial_{x}^{m-j} \gamma\right) \partial_{s}^{j} h,
$$

with $P_{m-1}$ a polynomial of degree at most $m-1$. A bound on $\left\|\partial_{x}^{\ell} \vec{\kappa}_{i}\right\|_{C^{0}(\bar{I})}$ follows from (6.23) taking $h=\vec{\kappa}_{i}$ and from bounds on $\left\|\partial_{S}^{\ell} \vec{\kappa}_{i}\right\|_{C^{0}(\bar{I})}$ (see (6.22)) and on $\left\|\partial_{x}^{\ell} \gamma_{i}\right\|_{C^{0}(\bar{I})}$. Thus it remains to estimate $\left\|\partial_{x}^{\ell} \gamma_{i}\right\|_{C^{0}(\bar{I})}$ for $\ell \in \mathbb{N}_{0}$. We start by showing that $\gamma_{i}=\left|\partial_{x} f_{i}\right|$ is uniformly bounded from above and below. Upon recalling (3.4) we see that each function $\gamma_{i}, i=1,2,3$, satisfies the following parabolic equation

$$
\partial_{t} \gamma_{i}=\left(\partial_{s} \varphi_{i}-\left\langle\vec{\kappa}_{i}, \vec{V}_{i}\right\rangle\right) \gamma_{i} .
$$

By regularity of the initial datum we have that $1 / c_{0} \leq \gamma_{i}(0) \leq c_{0}$ for some positive $c_{0}$. From the estimates given in (6.22), (6.7), and the uniform estimates for the tangential components and for the lengths of the curves, it follows that the coefficients $\left\|\partial_{S} \varphi_{i}\right\|_{C^{0}(\bar{I})}+\left\|\left\langle\vec{\kappa}_{i}, \vec{V}_{i}\right\rangle\right\|_{C^{0}(\bar{I})}$ in (6.24) are uniformly bounded and hence we infer that $1 / C \leq \gamma_{i} \leq C$, with $C$ having the same dependencies as the constant in (6.22) as well as $T$. In order to prove bounds on $\partial_{x}^{m} \gamma_{i}$ we proceed by induction. Let us assume that we have shown

$$
\left\|\partial_{x}^{j} \gamma_{i}\right\|_{C^{0}(\bar{I})} \leq C\left(m, \delta, n, T, \mathcal{L}\left(f_{k}\right), \mathcal{E}_{\lambda}\left(f_{k, 0}\right), f_{k, 0}, \lambda_{k}\right) \text { with } k=1,2,3 \text {, for } 0 \leq j \leq m
$$

$i=1,2,3$, and $m \in \mathbb{N}_{0}$. Choosing $h=\left\langle\vec{\kappa}_{i}, \vec{V}_{i}\right\rangle$ in (6.23), the induction assumption and (6.22) yield that

$$
\left\|\partial_{x}^{i}\left\langle\vec{\kappa}_{q}, \vec{V}_{q}\right\rangle\right\|_{C^{0}(\bar{I})} \leq C\left(m, \delta, n, T, \mathcal{L}\left(f_{j}\right), \varepsilon_{\lambda}\left(f_{j, 0}\right), f_{j, 0}, \lambda_{j}\right) \text { with } j=1,2,3,
$$

for all $0 \leq i \leq m+1, q=1,2$, 3. Differentiating (6.24) $(m+1)$-times with respect to $x$, and recalling that $\partial_{x}\left(\partial_{s} \varphi_{i}\right)=0$ by (6.7), we find

$$
\partial_{t} \partial_{x}^{m+1} \gamma_{q}=-\left\langle\vec{\kappa}_{q}, \vec{V}_{q}\right\rangle \partial_{x}^{m+1} \gamma_{q}-\sum_{\substack{i+j=m+1 \\ j \leq m}} c(i, j, m, q) \partial_{x}^{i}\left(\left\langle\vec{\kappa}_{q}, \vec{V}_{q}\right\rangle\right) \partial_{x}^{j} \gamma_{q},
$$

for some coefficients $c(i, j, m, q)$ and $q=1,2$, 3. Together with (6.25), (6.26) we derive

$$
\left|\sum_{\substack{i+j=m+1 \\ j \leq m}} c(i, j, m, q) \partial_{x}^{i}\left(\left\langle\vec{\kappa}_{q}, \vec{V}_{q}\right\rangle\right) \partial_{x}^{j} \gamma_{q}\right| \leq C,
$$


with $C=C\left(m, \delta, n, T, \mathcal{L}\left(f_{j}\right), \varepsilon_{\lambda}\left(f_{j, 0}\right), f_{j, 0}, \lambda_{j}\right.$, with $j=1,2,3$, which implies

$$
\left\|\partial_{x}^{m+1} \gamma_{i}\right\|_{C^{0}(\bar{I})} \leq C\left(m, \delta, n, T, \mathcal{L}\left(f_{j}\right), \mathcal{E}_{\lambda}\left(f_{j, 0}\right), f_{j, 0}, \lambda_{j}\right) \text { with } j=1,2,3,
$$

for $i=1,2,3$. Next note that (6.22) implies

$$
\sum_{i=1}^{3}\left\|\partial_{s}^{m} \vec{V}_{i}\right\|_{C^{0}(\bar{I})} \leq C\left(m, \delta, n, \mathcal{L}\left(f_{j}\right), \varepsilon_{\lambda}\left(f_{j, 0}\right), f_{j, 0}, \lambda_{j}\right) \text { with } j=1,2,3,
$$

which in turns gives uniform estimates for $\left\|\partial_{x}^{m} \vec{V}_{i}\right\|_{C^{0}(\bar{I})}, i=1,2,3$, in view of (6.23) and the bounds for the length elements and its derivatives.

Finally, since the points $P_{i}, i=1,2,3$ are fixed by the uniform bounds on the length, we derive that $\left\|f_{i}\right\|_{C^{0}}$ is uniformly bounded. This together with the uniform $C^{0}$-bounds on the curvature $\vec{\kappa}_{i}$, the velocity $\vec{V}_{i}, \gamma_{i}, \varphi_{i}$ (recall Lemma A.3, (6.7)) and all their derivatives, allow for a smooth extension of $f_{i}$ up to $t=T$.

Fifth Step: Long-time existence We want now to restart the flow at $t=T$ and by the short-time existence result get a contradiction to the maximality of $T$. To do that we have to verify that the network $f(T)$ satisfies the compatibility conditions required by Theorem C.1 and that the glueing of the curves across $t=T$ occurs in a smooth way. This requires some care. For clarity let us write down the strategy recalling also the major steps performed so far. Also, since several reparametrizations are necessary, let us distinguish again carefully between tangential components.

1. In Section 2.3 we have shown that given an initial three-network $\Gamma_{0}=\left\{f_{1,0}, f_{2,0}, f_{3,0}\right\}$ parametrized by constant speed and satisfying the assumptions of Theorem C.1, the geometric problem (1.10), (1.9), with $\varphi_{i}:=\varphi_{i}^{G}$ satisfying (6.6) (see also (2.6)), admits a short-time solution. Next we look at the maximal interval of existence $[0, T)$ for this problem.

2. If $T<\infty$ and Assumption 6.1 holds then Step 4 above shows that we can extend $f$ smoothly up to $T$. Hence we have regular smooth curves $f_{i} \in C^{\infty}([0, T] \times[0,1]), i=1,2,3$.

3. Next, we reparametrize each curve by constant speed via the diffeomorphisms

$$
\phi_{i}(t, x):=\frac{1}{\mathcal{L}\left(f_{i}(t)\right)} \int_{0}^{x}\left|\partial_{x} f_{i}(t, y)\right| d y, \quad(t, x) \in[0, T] \times[0,1] .
$$

Due to our assumptions on the initial datum, $\phi_{i}(0, x)=x$. Note that $\phi_{i}$ is smooth and a straightforward computation that uses integration by parts yields

$$
\begin{aligned}
\partial_{t} \phi_{i}(t, x)=- & \frac{1}{\mathcal{L}\left(f_{i}(t)\right)^{2}}\left(\varphi_{i}^{G}(t, 1)-\varphi_{i}^{G}(t, 0)-\int_{0}^{1}\left\langle\vec{V}_{i}, \vec{\kappa}_{i}\right\rangle d s\right) \int_{0}^{x}\left|\partial_{x} f_{i}(t, y)\right| d y \\
+ & \frac{1}{\mathcal{L}\left(f_{i}(t)\right)}\left(\varphi_{i}^{G}(t, x)-\varphi_{i}^{G}(t, 0)-\int_{0}^{x}\left\langle\vec{V}_{i}, \vec{\kappa}_{i}\right\rangle d s\right) \\
& \quad \text { for }(t, x) \in(0, T] \times[0,1] .
\end{aligned}
$$

Due to the regularity of $f_{i}$ we see that $\phi_{i} \in C^{\infty}([0, T] \times[0,1])$. Let $\psi_{i}(t, \cdot)$ be the inverse diffeomorphism for each $t$. Then, $\tilde{f}_{i}(t, x):=f_{i}\left(t, \psi_{i}(t, x)\right)$ is in $C^{\infty}([0, T] \times[0,1])$. Moreover, $\tilde{f}=\left(\tilde{f}_{1}, \tilde{f}_{2}, \tilde{f}_{3}\right)$ is solution in $C^{\infty}([0, T] \times[0,1])$ of

$$
\begin{aligned}
\partial_{t} \tilde{f}_{i}(t, x)= & \partial_{t} f_{i}\left(t, \psi_{i}(t, x)\right)+\partial_{x} f_{i}\left(t, \psi_{i}(t, x)\right) \partial_{t} \psi_{i}(t, x) \\
= & {\left[-\nabla_{S}^{2} \vec{\kappa}_{i}-\frac{1}{2}\left|\vec{\kappa}_{i}\right|^{2} \vec{\kappa}_{i}+\lambda_{i} \vec{\kappa}_{i}+\varphi_{i}^{G} \partial_{s} f_{i}\right]\left(t, \psi_{i}(t, x)\right) } \\
& \quad+\partial_{x} f_{i}\left(t, \psi_{i}(t, x)\right) \partial_{t} \psi_{i}(t, x) \\
= & {\left[-\nabla_{S}^{2} \vec{\kappa}_{i}-\frac{1}{2}\left|\vec{\kappa}_{i}\right|^{2} \vec{\kappa}_{i}+\lambda_{i} \vec{\kappa}_{i}+\left(\varphi_{i}^{G}+\left|\partial_{x} f_{i}\right| \partial_{t} \psi_{i}(t, x)\right) \partial_{s} f_{i}\right]\left(t, \psi_{i}(t, x)\right), }
\end{aligned}
$$




$$
=\left[-\nabla_{s}^{2} \overrightarrow{\tilde{\kappa}}_{i}-\frac{1}{2}\left|\overrightarrow{\tilde{\kappa}}_{i}\right|^{2} \overrightarrow{\tilde{\kappa}}_{i}+\lambda_{i} \overrightarrow{\tilde{\kappa}}_{i}+\varphi_{i}^{\star \star} \partial_{s} \tilde{f}_{i}\right](t, x)
$$

(where for the new tangential component $\varphi_{i}^{\star \star}$ we have that

$$
\varphi_{i}^{\star \star}=\varphi_{i}^{G}=\varphi_{i}^{\star} \quad \text { at } x=0,1
$$

and where $\overrightarrow{\tilde{\kappa}}_{i}$ denotes the curvature vector of the curve $\tilde{f}_{i}$ ) with boundary conditions

$$
\begin{array}{ll}
\tilde{f}_{i}(t, 1)=P_{i}, & \text { for all } t \in[0, T], i=1,2,3, \\
\tilde{f}_{1}(t, 0)=\tilde{f}_{2}(t, 0)=\tilde{f}_{3}(t, 0) & \text { for all } t \in[0, T], \\
\sum_{i=1}^{3}\left(\nabla_{s} \overrightarrow{\tilde{\kappa}}_{i}(t, 0)-\lambda_{i} \partial_{s} \tilde{f}_{i}(t, 0)\right)=0 & \text { for all } t \in[0, T],
\end{array}
$$

and

$$
\overrightarrow{\tilde{\kappa}}_{i}(t, 1)=\frac{\partial_{X}^{2} \tilde{f}_{i}}{\left|\partial_{x} \tilde{f}_{i}\right|^{2}}(t, 1)=0=\frac{\partial_{X}^{2} \tilde{f}_{i}}{\left|\partial_{x} \tilde{f}_{i}\right|^{2}}(t, 0)=\overrightarrow{\tilde{\kappa}}_{i}(t, 0)
$$

for all $t \in[0, T], i=1,2$, 3. Notice that here we use smoothness of the solution and the fact that $\tilde{f}_{i}, i=1,2,3$, are parametrized by constant speed to derive the boundary conditions on $[0, T]$.

4. Since we have a smooth solution at time $t=T$ the solution $f(T)$ satisfies the compatibility conditions for (6.28) and (6.29), (6.30). We want $f(T)$ to satisfy the compatibility conditions of the analytical problem treated in Appendix C. On a first sight the two problems differ by the choice of the tangential component and by the boundary condition.

5. Fundamental: the tangential components of all considered problems are equal at the boundary for any time. This holds also for their time-derivatives. Moreover, at the boundary the tangential components $\varphi_{i}^{\star \star}=\varphi_{i}^{G}=$ $\varphi_{i}^{\star}$ can be expressed in geometric terms.

6. Observation: On the equivalence of the compatibility conditions for $\frac{\partial_{x}^{2} f}{\left|\partial_{x} f\right|^{2}}=0$ and $\partial_{x}^{2} f=0$ for curves parametrized by constant speed.

At the boundary we observe:

Step 1: $\frac{\partial_{x}^{2} f}{\left|\partial_{x} f\right|^{2}}=0$ if and only if $\partial_{x}^{2} f=0$.

Step 2: Knowing that $\frac{\partial_{x}^{2} f}{\left|\partial_{x} f\right|^{2}}=0$ (and hence $\partial_{x}^{2} f=0$ ) and $\partial_{t} \frac{\partial_{x}^{2} f}{\left|\partial_{x} f\right|^{2}}=0$ we compute

$$
\partial_{t} \frac{\partial_{x}^{2} f}{\left|\partial_{x} f\right|^{2}}=0 \Rightarrow 0=\frac{\partial_{t} \partial_{x}^{2} f}{\left|\partial_{x} f\right|^{2}}+\partial_{x}^{2} f \partial_{t}\left(\frac{1}{\left|\partial_{x} f\right|^{2}}\right)=\frac{\partial_{t} \partial_{x}^{2} f}{\left|\partial_{x} f\right|^{2}} \Rightarrow \partial_{t} \partial_{x}^{2} f=0
$$

Viceversa, knowing that $\partial_{t} \partial_{x}^{2} f=0$ and $\partial_{x}^{2} f=0$ yields

$$
\partial_{t} \partial_{x}^{2} f=0 \Rightarrow \partial_{t} \frac{\partial_{x}^{2} f}{\left|\partial_{x} f\right|^{2}}=0 .
$$

One proceeds analogously for higher order derivatives.

7. Hence, at $t=T$ the compatibility conditions for the analytical problem (see Remark C.3) are satisfied.

8. We restart the flow at $f(T)$ via Theorem C.1 (hence solving the analytical problem) and get a smooth solution in some time interval $[T, T+\epsilon]$.

9. Reparametrize the solution in such a way that the tangential component linearly interpolates (using Lemma C.4 and choosing the initial diffeomorphism $\psi_{i}(x)=\phi_{i}(T, x)$ (see (6.27)), so that the original parametrization $f(T)$ is attained again)

10. We obtain a smooth solution of the geometric probelm (1.10), (1.9), with $\varphi_{i}:=\varphi_{i}^{G}$ satisfying (6.6), together with $f(T)$ as initial data on some interval $[T, T+\tilde{\epsilon}]$, for $0<\tilde{\epsilon}<\epsilon$. The latter is by construction a smooth extension of the geometric problem. This yields a contradiction, that is $T$ can not be finite.

Sixth Step: Sub-convergence The statement follows from an adaptation of the arguments depicted in [8, § 5 (Step nine)] to the present case. 
Remark 6.3. The long-time existence result can be extended to the case $\lambda_{i} \geq 0$ with just few modifications. Indeed, in order to derive the bounds on the curvatures and on the tangential components one needs only bounds on the lengths from below. The fact that $\lambda_{i}>0$ has been used in the proof above to derive a bound from above on the length. In the case $\lambda_{i} \geq 0$ since by (3.4) (see also Remark 3.3) the lengths grow at most linearly one has also $a$ bound from above on the length in finite time. This is sufficient to conclude the argument by contradiction. See [8, (5.14)] for a similar argument. The presence of the tangential component does not create any difficulty.

Remark 6.4. Our main result Theorem 1.1 remains valid (with smoothness guaranteed only away from $t=0$ ) also when the curves in the initial network $\Gamma_{0}$ are just $C^{4, \alpha}([0,1]), \alpha \in(0,1)$, and satisfy (when parametrized by constant speed) the compatibility condition of order one (see Remark C.3). Indeed, these assumptions are sufficient (see Remark C.2) to get a short time solution $f=\left(f_{1}, f_{2}, f_{3}\right)$ for $t \in[0, \varepsilon]$ of (C2), (C4) (and also of (1.8), (1.9)) that is instantaneously smooth. Moreover, at any $t \in(0, \varepsilon)$ compatibility conditions of any order are satisfied at the boundary. Hence this solution at some time $t \in(0, \varepsilon)$ is an admissible initial value to apply the same construction presented in Section 2.3 and for our main result.

Remark 6.5. We think that Assumption 6.1 is not just a technical assumption. In fact it is possible to picture situations where at least one of the requirements might stop holding true. For instance let us look at the condition imposed on the lengths of the curves. If we set $\lambda_{1}=\lambda_{2}=\lambda_{3}=1$ a network made up of three straight segments that meet at angles of $\frac{2}{3} \pi$ is a critical point for the energy functional. One thinks immediately at the "MercedezBenz symbol" but depending on the relative position of the points $P_{1}, P_{2}$ and $P_{3}$ it might be energetically better to have the junction point at one of the prescribed points. In this case, we will expect that along the elastic flow starting with an initial datum near to this configuration one of the curves collapses to a point.

Remark 6.6. For the sake of simplicity, we have considered so far only the case of a single triod. This gave us the opportunity to explore how to treat equations and boundary conditions, when either one endpoint of a curve is fixed or when it is connected to a triple junction. It is now possible to consider more complicated networks, composed of $m$ curves $(m \geq 3)$ meeting only in triple junctions and having at least one fixed point $P_{i}$ in space (which guarantees that the network doe not translate in space). For this type of configurations one can easily generalize our main result.

\section{A Supporting lemmas}

Lemma A.1. Let $a_{1}, \ldots, a_{n}$ be positive numbers and assume $0<i_{1}+\ldots+i_{n}<2$, where $i_{j}>0$ for all $j=1, \ldots, n$. Then for any $\epsilon>0$ we have

$$
a_{1}^{i_{1}} \cdot a_{2}^{i_{2}} \cdot \ldots \cdot a_{n}^{i_{n}} \leq \epsilon\left(a_{1}^{2}+\ldots a_{n}^{2}\right)+C_{\epsilon} .
$$

Proof. The proof goes by induction. The case $n=1$ is simply the standard Young inequality. Now suppose the claim holds for $n-1$. Then since by hypothesis we have $2\left(i_{2}+\ldots+i_{n}\right) /\left(2-i_{1}\right)<2$ we infer applying Young and the induction hypothesis that

$$
a_{1}^{i_{1}} \cdot a_{2}^{i_{2}} \cdot \ldots \cdot a_{n}^{i_{n}} \leq \epsilon a_{1}^{2}+C_{\epsilon}\left(a_{2}^{i_{2}} \cdot \ldots \cdot a_{n}^{i_{n}}\right)^{2 /\left(2-i_{1}\right)} \leq \epsilon a_{1}^{2}+C_{\epsilon} \delta\left(a_{2}^{2}+\ldots+a_{n}^{n}\right)+C_{\epsilon} C_{\delta}
$$

for any $\delta>0$. Choosing $\delta<\epsilon / C_{\epsilon}$ the claim follows. 
Lemma A.2. Let $a_{1}, \ldots, a_{n}$ be positive numbers such that $a_{i} \geq 1$ for any $i=1, \ldots, n$ and assume $0<i_{1}+$ $\ldots+i_{n}<\gamma$, where $i_{j}>0$ for all $j=1, \ldots, n$ and some $\gamma>0$. Then we have

$$
a_{1}^{i_{1}} \cdot a_{2}^{i_{2}} \cdot \ldots \cdot a_{n}^{i_{n}} \leq \sum_{i=1}^{n} a_{i}^{\gamma} .
$$

Proof. The proof follows by an induction argument over $n$ and uses the $(p, q)$-Young inequality. Induction start: for $n=1$ the claim follows since $a_{1} \geq 1$. Suppose the claim holds for some $n-1$. Then Young inequality gives (note that $\frac{\gamma}{i_{1}}>1$ )

$$
a_{1}^{i_{1}} \cdot a_{2}^{i_{2}} \cdot \ldots \cdot a_{n}^{i_{n}} \leq \frac{i_{1}}{\gamma} a_{1}^{\gamma}+\frac{\gamma-i_{1}}{\gamma}\left(a_{2}^{i_{2}} \cdot \ldots \cdot a_{n}^{i_{n}}\right)^{\frac{\gamma}{\gamma-i_{1}}} \leq a_{1}^{\gamma}+\left(a_{2}^{i_{2}} \cdot \ldots \cdot a_{n}^{i_{n}}\right)^{\frac{\gamma}{\gamma-i_{1}}}
$$

On the second term the induction hypothesis can be applied since $\sum_{j=2}^{n} \frac{i_{j} \gamma}{\gamma-i_{1}}<\gamma$.

Lemma A.3. Let $m \in \mathbb{N}$ and suppose that (6.13) and (6.14) hold. Let the tangential components be defined as in (6.6). Then we have that for any $r \in \mathbb{N}, r \leq m$

$$
\begin{aligned}
\left|\partial_{t}^{r} \varphi_{i}(t, x)\right| & \leq\left|\partial_{t}^{r} \varphi_{i}(t, 0)\right|+C, \quad\left|\frac{d^{r}}{d t^{r}} \mathcal{L}\left(f_{i}\right)\right| \leq C, \\
\left|\partial_{t}^{m+1} \varphi_{i}(t, x)\right| & \leq\left|\partial_{t}^{m+1} \varphi_{i}(t, 0)\right|+C\left(\left|\partial_{t}^{m} \varphi_{i}(t, 0)\right|+1\right), \\
\left|\partial_{s} \partial_{t}^{r} \varphi_{i}(t, x)\right| & \leq C\left(\left|\partial_{t}^{r} \varphi_{i}(t, 0)\right|+1\right) \text { and } \\
\left|\partial_{s}^{j} \partial_{t}^{r} \varphi_{i}(t, x)\right| & \leq C \text { for } j=2,3,4 .
\end{aligned}
$$

for any $(t, x) \in(0, T) \times[0,1]$. Here the constant $C$ has the same dependencies as the constants appearing in (6.13) and (6.14).

Proof. Recalling the definition (6.6) of $\varphi_{i}$, (6.7), (3.12), and (6.8) we can write

$$
\begin{aligned}
\partial_{s} \partial_{t} \varphi_{i}(t, x)= & -\frac{\partial_{t} \varphi_{i}(t, 0)}{\mathcal{L}\left(f_{i}\right)}+\frac{\varphi_{i}(t, 0)}{\mathcal{L}\left(f_{i}\right)^{2}}\left(-\varphi_{i}(t, 0)-\int_{I}\left\langle\vec{\kappa}_{i}, \vec{V}_{i}\right\rangle d s\right) \\
& -\frac{\varphi_{i}(t, 0)}{\mathcal{L}\left(f_{i}\right)}\left(\partial_{s} \varphi_{i}-\left\langle\vec{\kappa}_{i}, \vec{V}_{i}\right\rangle\right), \\
\partial_{s}^{m} \partial_{t} \varphi_{i}(t, x)= & \frac{\varphi_{i}(t, 0)}{\mathcal{L}\left(f_{i}\right)} \partial_{s}^{m-1}\left(\left\langle\vec{\kappa}_{i}, \vec{V}_{i}\right\rangle\right) \\
= & \frac{\varphi_{i}(t, 0)}{\mathcal{L}\left(f_{i}\right)} \sum_{\substack{[[a, b]] \leq[[m+1,2]] \\
c \leq m+1 \\
b \text { even }}} P_{b}^{a, c}\left(\vec{\kappa}_{i}\right) \text { for any } m \geq 2 .
\end{aligned}
$$

To get some induction argument going let us write

$$
\varphi_{i}(x, t)=\varphi_{i}(t, 0) B(t, x)
$$

with

$$
B(t, x):=1-\frac{1}{\mathcal{L}\left(f_{i}\right)} \int_{0}^{x}\left|\left(f_{i}\right)_{x}\right| d x=\frac{1}{\mathcal{L}\left(f_{i}\right)} \int_{x}^{1}\left|\left(f_{i}\right)_{x}\right| d x
$$

Obviously $0 \leq B(t, x) \leq 1$. For $m, k \in \mathbb{N}$ we have

$$
\begin{aligned}
\partial_{t}^{m} \varphi_{i}(x, t) & =\sum_{r=0}^{m}\left(\begin{array}{c}
m \\
r
\end{array}\right) \partial_{t}^{m-r} \varphi_{i}(t, 0) \partial_{t}^{r} B(t, x) \\
& =\partial_{t}^{m} \varphi_{i}(t, 0) B(t, x)+\sum_{r=1}^{m}\left(\begin{array}{c}
m \\
r
\end{array}\right) \partial_{t}^{m-r} \varphi_{i}(t, 0) \partial_{t}^{r} B(t, x),
\end{aligned}
$$




$$
\begin{aligned}
\partial_{s}^{k} \partial_{t}^{m} \varphi_{i}(x, t) & =\sum_{r=0}^{m}\left(\begin{array}{c}
m \\
r
\end{array}\right) \partial_{t}^{m-r} \varphi_{i}(t, 0) \partial_{s}^{k} \partial_{t}^{r} B(t, x) \\
& =\partial_{t}^{m} \varphi_{i}(t, 0) \partial_{s}^{k} B(t, x)+\sum_{r=1}^{m}\left(\begin{array}{c}
m \\
r
\end{array}\right) \partial_{t}^{m-r} \varphi_{i}(t, 0) \partial_{s}^{k} \partial_{t}^{r} B(t, x) .
\end{aligned}
$$

The term $B$ satisfies

$$
\begin{aligned}
& \mathcal{L}\left(f_{i}\right) B(t, x)=\int_{x}^{1}\left|\left(f_{i}\right)_{x}\right| d x, \\
& \mathcal{L}\left(f_{i}\right) \partial_{s} B(t, x)=-1, \quad \partial_{s}^{k} B(t, x)=0 \text { for any } k \geq 2, \\
& \mathcal{L}\left(f_{i}\right) \partial_{t} B(t, x)+B(t, x) \frac{d}{d t} \mathcal{L}\left(f_{i}\right)=\int_{x}^{1}\left(\partial_{s} \varphi_{i}-\left\langle\vec{\kappa}_{i}, \vec{V}_{i}\right\rangle\right) d s=-\varphi_{i}(t, x)-\int_{x}^{1}\left\langle\vec{\kappa}_{i}, \vec{V}_{i}\right\rangle d s, \\
& =-\varphi_{i}(t, x)+\int_{x}^{1} \sum_{\substack{[[a, b]] \leq[[2,2]] \\
c \leq 2 \\
b \text { even }}} P_{b}^{a, c}\left(\vec{\kappa}_{i}\right) d s, \\
& \mathcal{L}\left(f_{i}\right) \partial_{s} \partial_{t} B(t, x)+\partial_{s} B(t, x) \frac{d}{d t} \mathcal{L}\left(f_{i}\right)=-\partial_{s} \varphi_{i}(t, x)+\left\langle\vec{\kappa}_{i}, \vec{V}_{i}\right\rangle,
\end{aligned}
$$

so that

$$
\begin{aligned}
\mathcal{L}\left(f_{i}\right) \partial_{s}^{m} \partial_{t} B(t, x)= & \partial_{s}^{m-1}\left\langle\vec{\kappa}_{i}, \vec{V}_{i}\right\rangle \text { for } m \geq 2, \\
\mathcal{L}\left(f_{i}\right) \partial_{t}^{m} B(t, x)= & -\sum_{r=0}^{m-1}\left(\begin{array}{c}
m \\
r
\end{array}\right)\left({\frac{d^{m}}{d t}}^{m-r} \mathcal{L}\left(f_{i}\right)\right) \partial_{t}^{r} B(t, x)-\partial_{t}^{m-1} \varphi_{i}(t, x) \\
& +\partial_{t}^{m-1}\left(\int_{x}^{1} \sum_{\substack{[[a, b]] \leq[[2,2]] \\
c \leq 2 \\
b \text { even }}} P_{b}^{a, c}\left(\vec{\kappa}_{i}\right) d s\right), \\
\mathcal{L}\left(f_{i}\right) \partial_{s}^{k} \partial_{t}^{m} B(t, x)= & -\sum_{r=0}^{m-1}\left(\begin{array}{c}
m \\
r
\end{array}\right)\left(\frac{d}{d t}^{m-r} \mathcal{L}\left(f_{i}\right)\right) \partial_{s}^{k} \partial_{t}^{r} B(t, x)-\partial_{s}^{k} \partial_{t}^{m-1} \varphi_{i}(t, x) \\
& +\partial_{s}^{k} \partial_{t}^{m-1}\left(\int_{x}^{1}\left\langle\vec{\kappa}_{i}, \vec{V}_{i}\right\rangle d s\right) .
\end{aligned}
$$

Now from (3.12) we know that

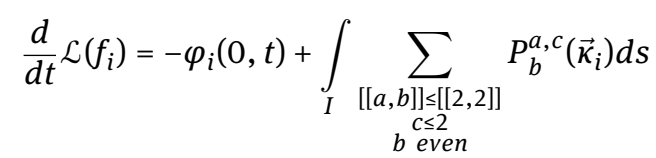

so that by (3.18), (3.4), and an induction argument that uses repeatedly (for $\psi$ a scalar quantity)

$$
\frac{d}{d t}\left(\int_{I} \psi d s\right)=\int_{I}\left(\psi_{t}-\psi\langle\vec{\kappa}, \vec{V}\rangle\right) d s+\varphi_{s} \int_{I} \psi d s
$$

we infer for $m \in \mathbb{N}$,

$$
\begin{aligned}
\frac{d^{m+1}}{d t^{m+1}} \mathcal{L}\left(f_{i}\right)= & -\partial_{t}^{m} \varphi_{i}(0, t) \\
& +\tilde{p}\left(\varphi_{i}(0), \ldots, \partial_{t}^{m-1} \varphi_{i}(0), \frac{1}{\mathcal{L}\left(f_{i}\right)}, \frac{d}{d t} \mathcal{L}\left(f_{i}\right), . ., \frac{d^{m-1}}{d t^{m-1}} \mathcal{L}\left(f_{i}\right), r_{m}(t)\right),
\end{aligned}
$$


with

$$
r_{m}(t):=\int_{I} \sum_{\substack{[[a, b]] \leq[2+4 m, 2]] \\
c \leq 2+4 m \\
b \text { even }}} P_{b}^{a, c}\left(\vec{\kappa}_{i}\right) d s+\int_{I} \sum_{\beta \in S_{4 m}^{m-1}} \prod_{l=0}^{m-1}\left(\varphi_{i}^{(l)}\right)^{\beta_{l}} \sum_{\begin{array}{c}
{[[a, b]] \leq[[2+4 m-|\beta|, 2]]} \\
c \leq 2+4 m-|\beta| \\
b \text { even }
\end{array}} P_{b}^{a, c}\left(\vec{\kappa}_{i}\right) d s
$$

and where $\tilde{p}$ is a polynomial in the listed variables. Similarly

$$
\partial_{t}^{m} \int_{\substack{x[a, b]] \leq[[2,2]] \\ c \leq 2 \\ b \text { even }}}^{1} P_{b}^{a, c}\left(\vec{\kappa}_{i}\right) d s=\tilde{p}\left(\varphi_{i}(0), \ldots, \partial_{t}^{m-1} \varphi_{i}(0), \frac{1}{\mathcal{L}\left(f_{i}\right)}, \frac{d}{d t} \mathcal{L}\left(f_{i}\right), \ldots, \frac{d^{m-1}}{d t^{m-1}} \mathcal{L}\left(f_{i}\right), r_{m}^{x}(t)\right)
$$

where

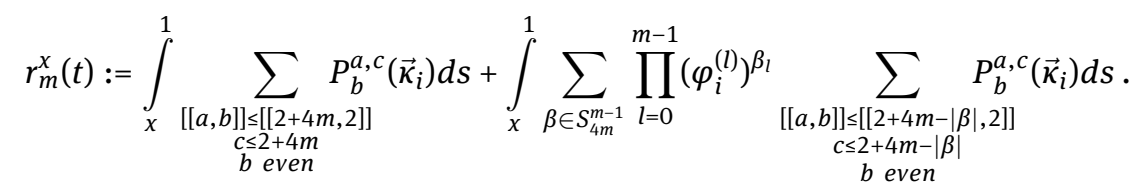

Finally observe that for $m \in \mathbb{N} \cup\{0\}$

$$
\begin{aligned}
& \partial_{s} \partial_{t}^{m}\left(\int_{x}^{1} \sum_{\substack{[[a, b]] \leq[[2,2]] \\
c \leq 2 \\
b \text { even }}} P_{b}^{a, c}\left(\vec{\kappa}_{i}\right) d s\right) \\
& =\tilde{p}\left(\varphi_{i}(0), . ., \partial_{t}^{m-1} \varphi_{i}(0), \frac{1}{\mathcal{L}\left(f_{i}\right)}, \frac{d}{d t} \mathcal{L}\left(f_{i}\right), . ., \frac{d^{m-1}}{d t^{m-1}} \mathcal{L}\left(f_{i}\right), R_{m}(t, x)\right)
\end{aligned}
$$

where

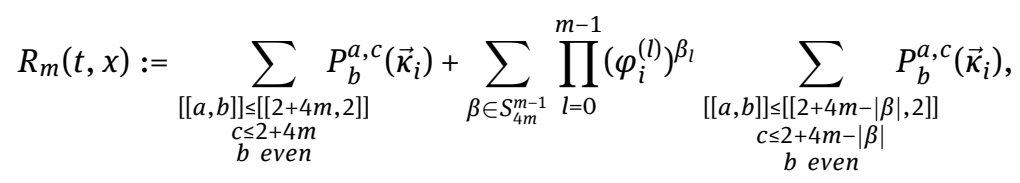

and more generaly for $k \in \mathbb{N}$

$$
\begin{aligned}
\partial_{s}^{k} \partial_{t}^{m}\left(\int_{x} \sum_{\substack{[[a, b]] \leq[[2,2]] \\
b \leq 2}} P_{b}^{a, c}\left(\vec{\kappa}_{i}\right) d s\right) \\
\quad=\tilde{p}\left(\varphi_{i}(0), . ., \partial_{t}^{m-1} \varphi_{i}(0), \frac{1}{\mathcal{L}\left(f_{i}\right)}, \frac{d}{d t} \mathcal{L}\left(f_{i}\right), . ., \frac{d^{m-1}}{d t^{m-1}} \mathcal{L}\left(f_{i}\right), \partial_{s}^{k-1} R_{m}(t, x)\right)
\end{aligned}
$$

with

$$
\begin{aligned}
\partial_{s}^{k-1} R_{m}(t, x):= & \tilde{p}\left(\sum_{\substack{[[a, b]] \leq[[2+4 m+k-1,2]] \\
c \leq 2+4 m+k-1 \\
b \text { even }}} P_{b}^{a, c}\left(\vec{\kappa}_{i}\right),\right. \\
& \left.\varphi_{i}(t, x), \partial_{s} \varphi_{i}(t, x), \ldots, \partial_{s}^{k-1} \varphi_{i}(t, x), . ., \varphi_{i}^{(m-1)}(t, x), . ., \partial_{s}^{k-1} \varphi_{i}^{(m-1)}(t, x)\right) .
\end{aligned}
$$

The first three claims follows now by an induction argument. More precisely: for $m=1$ starting from

$$
|B(t, x)| \leq C, \quad\left|\varphi_{i}(t, x)\right| \leq\left|\varphi_{i}(t, 0)\right| \leq C
$$

and using the expression for the derivatives of $\mathcal{L}\left(f_{i}\right), B$, and $\varphi_{i}(x, t)$ we first derive

$$
\left|\frac{d}{d t} \mathcal{L}\left(f_{i}\right)\right| \leq C, \quad\left|\partial_{t} B(t, x)\right| \leq C, \quad\left|\partial_{t} \varphi_{i}(x, t)\right| \leq\left|\partial_{t} \varphi_{i}(t, 0)\right|+C
$$


by (6.14), (6.13), Lemma 3.11 (with $\ell=4 \mathrm{~m}$ ), and the uniform control of the length from above and below. Then assuming that

$$
\left|\frac{d^{r}}{d t^{r}} \mathcal{L}\left(f_{i}\right)\right| \leq C, \quad\left|\partial_{t}^{r} B(t, x)\right| \leq C, \quad\left|\partial_{t}^{r} \varphi_{i}(x, t)\right| \leq\left|\partial_{t}^{r} \varphi_{i}(t, 0)\right|+C
$$

holds for some $1 \leq r<m$, we derive again by (6.14), (6.13) and using Lemma 3.11 (with $\ell=4 m$ ) that

$$
\left|\frac{d^{r+1}}{d t^{r+1}} \mathcal{L}\left(f_{i}\right)\right| \leq C, \quad\left|\partial_{t}^{r+1} B(t, x)\right| \leq C, \quad\left|\partial_{t}^{r+1} \varphi_{i}(t, x)\right| \leq\left|\partial_{t}^{r+1} \varphi_{i}(t, 0)\right|+C,
$$

respectively

$$
\begin{aligned}
&\left|\frac{d^{m+1}}{d t^{m+1}} \mathcal{L}\left(f_{i}\right)\right| \leq\left|\partial_{t}^{m} \varphi_{i}(t, 0)\right|+C, \quad\left|\partial_{t}^{m+1} B(t, x)\right| \leq C\left(\left|\partial_{t}^{m} \varphi_{i}(t, 0)\right|+1\right), \\
&\left|\partial_{t}^{m+1} \varphi_{i}(t, x)\right| \leq\left|\partial_{t}^{m+1} \varphi_{i}(t, 0)\right|+C\left(\left|\partial_{t}^{m} \varphi_{i}(t, 0)\right|+1\right) .
\end{aligned}
$$

Next, observe that from (A1), we infer (using also the bounds derived above for the derivative of the length functional)

$$
\begin{aligned}
& \left|\partial_{s} \partial_{t} \varphi_{i}(t, x)\right| \leq C\left(\left|\partial_{t} \varphi_{i}(t, 0)\right|+\left|\sum_{\substack{[[a, b]] \leq[[2,2]] \\
c \leq 2 \\
b \text { even }}} P_{b}^{a, c}\left(\vec{\kappa}_{i}\right)\right|\right) \leq C\left(\left|\partial_{t} \varphi_{i}(t, 0)\right|+C\right), \\
& \left|\partial_{s} \partial_{t} B(t, x)\right| \leq C,
\end{aligned}
$$

where we have used Lemma 3.7, Lemma 3.11, and (6.13). Next we infer using (A4) and (6.14) that

$$
\begin{aligned}
& \left|\partial_{s} \partial_{t}^{2} B(t, x)\right| \leq C\left(\left|\partial_{t} \varphi_{i}(t, 0)\right|+1\right) \leq C, \\
& \left|\partial_{s} \partial_{t}^{2} \varphi_{i}(t, x)\right| \leq C\left(\left|\partial_{t}^{2} \varphi_{i}(t, 0)\right|+\left|\partial_{t} \varphi_{i}(t, 0)\right|+1\right) \leq C\left(\left|\partial_{t}^{2} \varphi_{i}(t, 0)\right|+1\right) .
\end{aligned}
$$

Repeating the same arguments inductively we obtain the fourth claim in the lemma. Next, we observe from (A2), (A3), Lemma 3.7, Lemma 3.11, and (6.13) that

$$
\left|\partial_{s}^{k} \partial_{t} \varphi_{i}(t, x)\right|=\left|\frac{\varphi_{i}(t, 0)}{\mathcal{L}\left(f_{i}\right)} \sum_{\substack{[[a, b]] \leq[[k+1,2]] \\ c k+1 \\ b \text { even }}} P_{b}^{a, c}\left(\vec{\kappa}_{i}\right)\right| \leq C, \quad\left|\partial_{s}^{k} \partial_{t} B(t, x)\right| \leq C \text { for } k=2,3,4 .
$$

The final three claims of the lemma are again proved using an induction arguments and employing all estimates achieved so far: it is important that one proves the claim first for $k=2$, then $k=3$ and finally $k=4$.

Lemma A.4. Let $\varphi_{i}$ be the tangential component in (1.10), and $j, p \in \mathbb{N}_{0}$. Let $S_{p}^{j}$ be defined as in (4.4). Then

$$
\begin{aligned}
& \text { (i) } \quad \varphi_{i} \sum_{\beta \in S_{p}^{j}} \prod_{l=0}^{j}\left(\varphi_{i}^{(l)}\right)^{\beta_{l}} \sum_{\begin{array}{c}
{[[a, b]] \leq[[k-|\beta|, B]]} \\
c \leq k-|\beta| \\
b \text { odd }
\end{array}} P_{b}^{a, c}\left(\vec{\kappa}_{i}\right)=\sum_{\beta \in S_{p+3}^{j}} \prod_{l=0}^{j}\left(\varphi_{i}^{(l)}\right)^{\beta_{l}} \sum_{\begin{array}{c}
{[[a, b]] \leq[[3+k-|\beta|, B]]} \\
c \leq 3+k-|\beta| \\
b \text { odd }
\end{array}} P_{b}^{a, c}\left(\vec{\kappa}_{i}\right) \text {, } \\
& \text { (ii) } \partial_{t} \sum_{\beta \in S_{p}^{j}} \prod_{l=0}^{j}\left(\varphi_{i}^{(l)}\right)^{\beta_{l}}=\sum_{\beta \in S_{p+4}^{j+1}} \prod_{l=0}^{j+1} c_{\beta_{l}}\left(\varphi_{i}^{(l)}\right)^{\beta_{l}} \text { for some constants } c_{\beta_{l}} \text {, }
\end{aligned}
$$

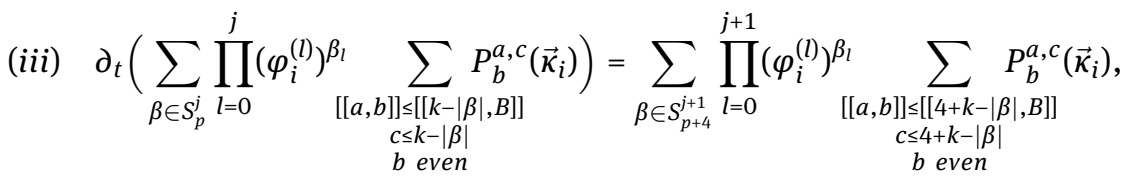

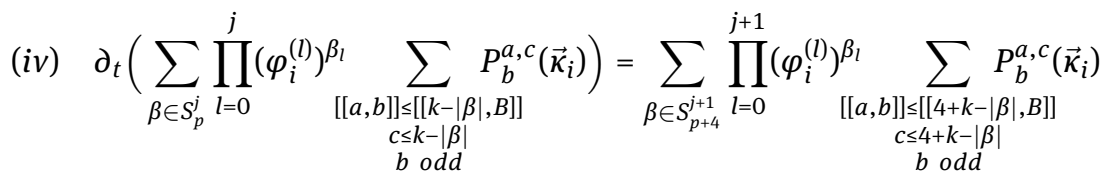




$$
+\partial_{s} f \sum_{\beta \in S_{p}^{j}} \prod_{l=0}^{j}\left(\varphi_{i}^{(l)}\right)^{\beta_{l}} \sum_{\substack{[[a, b]] \leq[[3+k-|\beta|, B+1]] \\ c \leq 3+k-|\beta| \\ b \text { even }}} P_{b}^{a, c}\left(\vec{\kappa}_{i}\right)
$$

at the points where $\vec{\kappa}_{i}=0$, in particular at the boundary.

Proof. Observe that

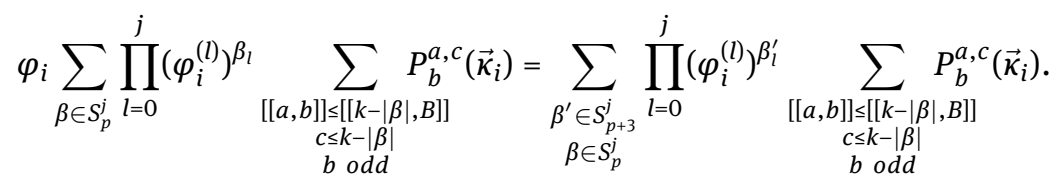

Since $\left|\beta^{\prime}\right|=|\beta|+3$, the proof of (i) is obtained from replacing $\beta$ by $\beta^{\prime}$ in the term $\sum_{[[a, b]] \leq[[k-|\beta|, B]]} P_{b}^{a, c}\left(\vec{\kappa}_{i}\right)$. The $c \leq k-|\beta|$
$b$ odd

proofs of (ii) is straightforward by using the defition of the notation $S_{p}^{j}$ in (4.4). The proofs of (iii) and (iv) are also straightforward, by applying (i), (ii) above, and (3.20) and (3.19).

\section{B Proofs of technical lemmas}

\section{B.1 Proof of Lemma 3.6}

Proof of (3.15) in Lemma 3.6. Since $P_{v}^{\mu, d}$ is linear combination of terms of the type

$$
\left\langle\nabla_{S}^{i_{1}} \vec{\kappa}, \nabla_{S}^{i_{2}} \vec{\kappa}\right\rangle \ldots\left\langle\nabla_{S}^{i_{v-2}} \vec{\kappa}, \nabla_{S}^{i_{v-1}} \vec{\kappa}\right\rangle \nabla_{S}^{i_{v}} \vec{\kappa}
$$

with $i_{1}+\cdots+i_{v}=\mu$ and $\max \left\{i_{j}\right\} \leq d$, by Leibnitz's rule we need to understand terms of the kind

$$
\left\langle\nabla_{s}^{i_{1}} \vec{\kappa}, \nabla_{s}^{i_{2}} \vec{\kappa}\right\rangle \ldots\left\langle\nabla_{t} \nabla_{s}^{i_{j}} \vec{\kappa}, \ldots\right\rangle \ldots\left\langle\nabla_{s}^{i_{v-2}} \vec{\kappa}, \nabla_{s}^{i_{v-1}} \vec{\kappa}\right\rangle \nabla_{s}^{i_{v}} \vec{\kappa}
$$

for $j \in\{1, \ldots, v-1\}$ or

$$
\left\langle\nabla_{S}^{i_{1}} \vec{\kappa}, \nabla_{S}^{i_{2}} \vec{\kappa}\right\rangle \ldots\left\langle\nabla_{S}^{i_{v-2}} \vec{\kappa}, \nabla_{S}^{i_{v-1}} \vec{\kappa}\right\rangle \nabla_{t} \nabla_{s}^{i_{v}} \vec{\kappa}
$$

with as before $i_{1}+\cdots+i_{v}=\mu$ and $\max \left\{i_{k}\right\} \leq d$. By Lemma 3.5 we have

$$
\nabla_{t} \nabla_{s}^{i_{j}} \vec{\kappa}=\varphi \nabla_{s}^{i_{j}+1} \vec{\kappa}+\sum_{\substack{[[a, b]] \leq\left[\left[4+i_{j}, 1\right]\right] \\ c \leq 4+i_{j} \\ b \in[1,5] \text { odd }}} P_{b}^{a, c}(\vec{\kappa})+\lambda \sum_{\substack{[[a, b]] \leq\left[\left[2+i_{j}, 1\right]\right] \\ c \leq 2+i_{j} \\ b \in[1,3] \text { odd }}} P_{b}^{a, c}(\vec{\kappa}) .
$$

It follows that

$$
\begin{aligned}
& \left\langle\nabla_{s}^{i_{1}} \vec{\kappa}, \nabla_{s}^{i_{2}} \vec{\kappa}\right\rangle \ldots\left\langle\nabla t \nabla_{s}^{i_{j}} \vec{\kappa}, . .\right\rangle \ldots\left\langle\nabla_{s}^{i_{v-2}} \vec{\kappa}, \nabla_{s}^{i_{v-1}} \vec{\kappa}\right\rangle \nabla_{s}^{i_{v}} \vec{\kappa} \\
& =\sum_{\substack{[[a, b]] \leq[[\mu+4, v]] \\
c \leq 4+d \\
b \in[v, v+4], o d d}} P_{b}^{a, c}(\vec{\kappa})+\lambda \sum_{\substack{[[a, b]] \leq[[\mu+2, v]] \\
c \leq d+2 \\
b \in[v, 2+v], o d d}} P_{b}^{a, c}(\vec{\kappa})+\varphi P_{v}^{\mu+1, \max \left\{d, i_{j}+1\right\}}(\vec{\kappa})
\end{aligned}
$$

for any $j \in\{1, \ldots, v-1\}$ and the same formula holds for the term in (B1). We get

$$
\nabla_{t} P_{v}^{\mu, d}(\vec{\kappa})=\sum_{\substack{[[a, b]] \leq[[\mu+4, v]] \\ c \leq 4+d \\ b \in[v, v+4], \text { odd }}} P_{b}^{a, c}(\vec{\kappa})+\lambda \sum_{\substack{[[a, b]] \leq[[\mu+2, v]] \\ c \leq 2+d \\ b \in[v, 2+v], \text { odd }}} P_{b}^{a, c}(\vec{\kappa})+\varphi P_{v}^{\mu+1, d+1,(\vec{\kappa}),}
$$

and the claim follows. 
Proof of (3.16) in Lemma 3.6. Next we observe that formula (B2) implies that

$$
\begin{aligned}
& \nabla_{t} \sum_{\substack{[[a, b]] \leq[[A, B]] \\
c \leq C \\
b \in[N, M], \text { odd }}} P_{b}^{a, c}(\vec{\kappa}) \\
& =\sum_{\substack{[[a, b]] \leq[[A, B]] \\
c \leq C \\
b \in[N, M], \text { odd }}}\left(\sum_{\substack{[[\alpha, \beta]] \leq[[a+4, b]] \\
\gamma \leq 4+c \\
\beta \in[b, b+4], \text { odd }}} P_{\beta}^{\alpha, \gamma}(\vec{\kappa})+\lambda \sum_{\substack{[[\alpha, \beta]] \leq[[a+2, b]] \\
\gamma \leq 2+c \\
\beta \in[b, 2+b], \text { odd }}} P_{\beta}^{\alpha, \gamma}(\vec{\kappa})\right. \\
& \left.+\varphi P_{b}^{a+1, c+1}(\vec{\kappa})\right) \\
& =\sum_{\substack{[[a, b]] \leq[[A+4, B]] \\
c \leq C+4 \\
b \in[N, M+4], \text { odd }}} P_{b}^{a, c}(\vec{\kappa})+\lambda \sum_{\substack{[[a, b]] \leq[[A+2, B]] \\
c \leq C+2 \\
b \in[N, M+2], \text { odd }}} P_{b}^{a, c}(\vec{\kappa})+\varphi \sum_{\substack{[[a, b]] \leq[[A+1, B]] \\
c \leq C+1 \\
b \in[N, M], \text { odd }}} P_{b}^{a, c}(\vec{\kappa}) .
\end{aligned}
$$

Proof of (3.17) and (3.18) in Lemma 3.6. The proof of (3.17) follows immediately from observing that $\partial_{t} P_{v}^{\mu, d}(\vec{\kappa})=$ $\nabla_{t} P_{v}^{\mu, d}(\vec{\kappa})+\left(\partial_{s} f\right)\left\langle P_{v}^{\mu, d}(\vec{\kappa}), \partial_{t} \tau\right\rangle$ and using (3.6) and (3.15). Equation (3.18) is derived with similar arguments employed for the proof of (3.15).

Proof of (3.19) and (3.20) in Lemma 3.6. The statements follow from (3.17) and (3.18).

\section{B.2 Proof of parts of Lemma 4.2}

Proof of (4.3) in Lemma 4.2. Based on (4.2), the proof follows from an induction argument. Suppose that (4.3) holds for some $m \in \mathbb{N}$ bigger than or equal to 2 . Then we take the covariant derivatives, $\nabla_{t}$, of (4.3). The lefthand side is simply obtained from using Lemma 3.5,

$$
\nabla_{t} \nabla_{s}^{4 m} \vec{\kappa}_{i}=-\nabla_{s}^{4+4 m} \vec{\kappa}_{i}+\varphi_{i} \nabla_{s}^{1+4 m} \vec{\kappa}_{i}+\sum_{\substack{[[a, b]] \leq[[2+4 m, 3]] \\ c \leq 2+4 m \\ b \text { odd }}} P_{b}^{a, c}\left(\vec{\kappa}_{i}\right) .
$$

By applying (3.16) and Lemma A.4, the right-hand side is

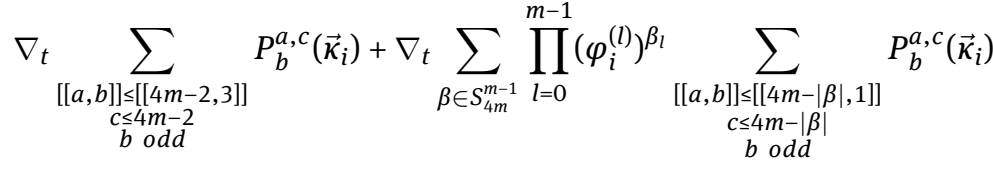

$$
\begin{aligned}
& =\sum_{\substack{[[a, b]] \leq[[2+4 m, 3]] \\
c \leq 2+4 m \\
b \text { odd }}} P_{b}^{a, c}\left(\vec{\kappa}_{i}\right)+\varphi_{i} \sum_{\substack{[[a, b]] \leq[[4 m-1,3]] \\
c \leq 4 m-1 \\
b \text { odd }}} P_{b}^{a, c}\left(\vec{\kappa}_{i}\right) \\
& +\sum_{\beta \in S_{4+4 m}^{m}} \prod_{\substack{l=0 \\
l}}^{m}\left(\varphi_{i}^{(l)}\right)^{\beta_{l}} \sum_{\substack{[[a, b]] \leq[[4 m+4-|\beta|, 1]] \\
c \leq 4 m+4-|\beta| \\
b \text { odd }}} P_{b}^{a, c}\left(\vec{\kappa}_{i}\right)
\end{aligned}
$$

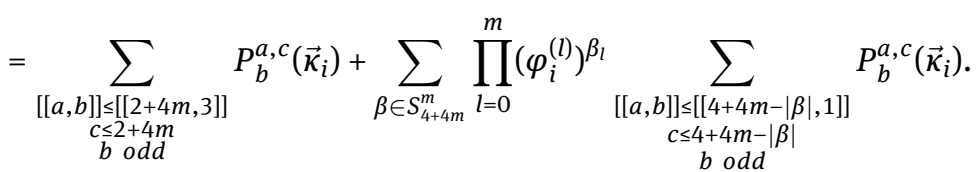

From (B3) and (B4), we finish the induction argument since (4.3) holds as $m$ therein is replaced by $m+1$.

Proof of (4.7) in Lemma 4.2. Besides (4.5) and (4.6), the following also holds by similar arguments,

$$
\sum_{i=1}^{3} \nabla_{s}^{13} \vec{\kappa}_{i}=\sum_{i=1}^{3}\left(\sum_{\substack{[[a, b]] \leq[[11,3]] \\ c \leq 11 \\ b \text { odd }}} P_{b}^{a, c}\left(\vec{\kappa}_{i}\right)+\varphi_{i} \sum_{\substack{[[a, b]] \leq[110,1]] \\ c \leq 10 \\ b \text { odd }}} P_{b}^{a, c}\left(\vec{\kappa}_{i}\right)+\varphi_{i}^{2} \sum_{\substack{[[a, b]] \leq[[7,1]] \\ c \leq 7 \\ b \text { odd }}} P_{b}^{a, c}\left(\vec{\kappa}_{i}\right)\right.
$$




$$
\begin{aligned}
& +\varphi_{i}^{3} \sum_{\substack{[[a, b]] \leq[[4,1]] \\
c \leq 4 \\
b \text { odd }}} P_{b}^{a, c}\left(\vec{\kappa}_{i}\right)+\partial_{t} \varphi_{i} \sum_{\substack{[[a, b]] \leq[[6,1]] \\
c \leq 6 \\
b \text { odd }}} P_{b}^{a, c}\left(\vec{\kappa}_{i}\right) \\
& +\varphi_{i} \partial_{t} \varphi_{i} \sum_{\substack{[[a, b]] \leq[[3,1]] \\
c \leq 3 \\
b \text { odd }}} P_{b}^{a, c}\left(\vec{\kappa}_{i}\right)+\partial_{t}^{2} \varphi_{i} \sum_{\substack{[[a, b]] \leq[[2,1]] \\
c \leq 2 \\
b \text { odd }}} P_{b}^{a, c}\left(\vec{\kappa}_{i}\right) \\
& +\partial_{s} f_{i}\left[\sum_{\substack{[[a, b]] \leq[[12,2]] \\
c \leq 11 \\
b \text { even }}} P_{b}^{a, c}\left(\vec{\kappa}_{i}\right)+\varphi_{i} \sum_{\substack{[[a, b]] \leq[[9,2]] \\
c \leq 8 \\
b \text { even }}} P_{b}^{a, c}\left(\vec{\kappa}_{i}\right)\right. \\
& \left.\left.+\varphi_{i}^{2} \sum_{\substack{[[a, b]] \leq[[6,2]] \\
c \leq 5 \\
b \text { even }}} P_{b}^{a, c}\left(\vec{\kappa}_{i}\right)+\partial_{t} \varphi_{i} \sum_{\substack{[[a, b]] \leq[[5,2]] \\
c \leq 4 \\
b \text { even }}} P_{b}^{a, c}\left(\vec{\kappa}_{i}\right)\right]\right),
\end{aligned}
$$

that is (4.7) for $m=2$. In these computations we use that the term multiplying $\varphi_{i}$ in the tangential component of (3.19) vanishes at the boundary since there $\vec{\kappa}_{i}=0$.

The proof of (4.7) follows then from an induction argument. Since (4.5), (4.6), and (B5) are the cases of $m=0,1,2$ in (4.7), we suppose that (4.7) holds for some $m \in \mathbb{N}_{0}$. Then we take the partial differentiation, $\partial_{t}$, of (4.7). By using Lemma 3.5, (3.7), and the fact that $\vec{\kappa}_{i}=0$ we obtain for the left-hand side,

$$
\begin{gathered}
\sum_{i=1}^{3} \partial_{t} \nabla_{s}^{5+4 m} \vec{\kappa}_{i}=\sum_{i=1}^{3}\left(-\nabla_{s}^{9+4 m} \vec{\kappa}_{i}+\varphi_{i} \nabla_{s}^{6+4 m} \vec{\kappa}_{i}+\sum_{\substack{[[a, b]] \leq[[7+4 m, 3]] \\
c \leq 7+4 m \\
b \text { odd }}} P_{b}^{a, c}\left(\vec{\kappa}_{i}\right)\right. \\
\left.+\partial_{s} f_{i}\left[\sum_{\substack{[[a, b]] \leq[[8+4 m, 2]] \\
c \leq 5+4 m \\
b \text { even }}} P_{b}^{a, c}\left(\vec{\kappa}_{i}\right)\right]\right) .
\end{gathered}
$$

By applying (3.19), (3.20), (3.6), Lemma A.4 and using the fact that $\vec{\kappa}_{i}=0$ the right-hand-side is equal to

$$
\begin{aligned}
& \sum_{i=1}^{3}\left\{\left[\sum_{\substack{[[a, b]] \leq[[7+4 m, 3]] \\
c \leq 7+4 m \\
b \text { odd }}} P_{b}^{a, c}\left(\vec{\kappa}_{i}\right)+\varphi_{i} \sum_{\substack{[[a, b]] \leq[[4+4 m, 3]] \\
c \leq 4+4 m \\
b \text { odd }}} P_{b}^{a, c}\left(\vec{\kappa}_{i}\right)\right]\right. \\
& +\partial_{s} f_{i}\left[\sum_{\substack{[[a, b]] \leq[[6+4 m, 4]] \\
c \leq 3+4 m \\
b \text { even }}} P_{b}^{a, c}\left(\vec{\kappa}_{i}\right)\right] \\
& +\sum_{\beta \in S_{8+4 m}^{m+1}} \prod_{\substack{l=0 \\
c \leq 5+4(m+1)-|\beta| \\
b \text { odd }}}^{m+1}\left(\varphi_{i}^{(l)}\right)^{\beta_{l}} \sum_{\substack{[[a, b]] \leq[[5+4(m+1)-|\beta|, 1] \\
c \leq 4}}^{a, c}\left(\vec{\kappa}_{i}\right) \\
& +\partial_{s} f_{i}\left[\sum_{\beta \in S_{4+4 m}^{m}} \prod_{\substack{l=0 \\
m}}^{m}\left(\varphi_{i}^{(l)}\right)^{\beta_{l}} \sum_{\substack{[a, b]] \leq[[4+4(m+1)-|\beta|, 2]] \\
c \leq 4+4(m+1)-|\beta| \\
b \text { even }}} P_{b}^{a, c}\left(\vec{\kappa}_{i}\right)\right] \\
& +\partial_{s} f_{i}\left[\sum_{\substack{[[a, b]] \leq[[8+4 m, 2]] \\
c \leq 7+4 m \\
b \text { even }}} P_{b}^{a, c}\left(\vec{\kappa}_{i}\right)+\varphi_{i} \sum_{\substack{[[a, b]] \leq[15+4 m, 2]] \\
c \leq 4+4 m \\
b \text { even }}} P_{b}^{a, c}\left(\vec{\kappa}_{i}\right)\right] \\
& +\partial_{s} f_{i}\left[\sum_{\beta \in S_{4+4 m}^{m}} \prod_{\substack{l=0 \\
c \leq 3+4(m+1)-|\beta| \\
b \text { even }}}\left(\varphi_{i}^{(l)}\right)^{\beta_{l}} P_{b}^{a, c} \sum_{\substack{[[a, b]] \leq[[4+4(m+1)-|\beta|, 2]] \\
c \leq 4}} \vec{\kappa}_{i}\right)
\end{aligned}
$$

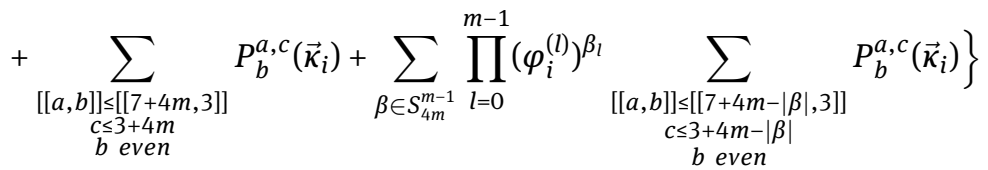




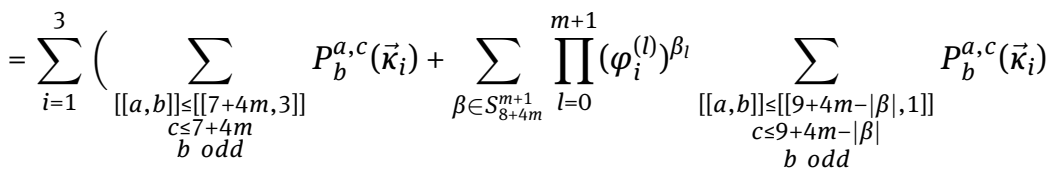

$$
\begin{aligned}
& \left.+\partial_{s} f_{i}\left[\sum_{\substack{[[a, b]] \leq[[8+4 m, 2]] \\
c \leq 7+4 m \\
b \text { even }}} P_{b}^{a, c}\left(\vec{\kappa}_{i}\right)+\sum_{\beta \in S_{4+4 m}^{m}} \prod_{\substack{l=0 \\
l[a, b]] \leq[[8+4 m-|\beta|, 2]] \\
c \leq 7+4 m-|\beta| \\
b \text { even }}}^{m}\left(\varphi_{i}^{(l)}\right)^{\beta_{l}} P_{b}^{a, c}\left(\vec{\kappa}_{i}\right)\right]\right) .
\end{aligned}
$$

The proof follows from using (B6) and (B7).

\section{Short-time existence result}

For the sake of the reader we collect here the necessary statements on short-time existence, that we need for our proof of long-time existence. All results and considerations listed below can be found in details in [6], where the problem of short-time existence is analyzed with great care.

To obtain a short-time existence result, it is crucial to choose a tangential component $\varphi_{i}$ in (1.10), that factors out the degeneracy of the geometric problem. By taking $\varphi_{i}:=\varphi_{i}^{\star}$ with

$$
\begin{aligned}
\varphi_{i}^{\star}= & -\left\langle\frac{\partial_{x}^{4} f_{i}}{\left|\partial_{x} f_{i}\right|^{5}}, \partial_{x} f_{i}\right\rangle+10 \frac{\left\langle\partial_{x}^{2} f_{i}, \partial_{x} f_{i}\right\rangle}{\left|\partial_{x} f_{i}\right|^{7}}\left\langle\partial_{x}^{3} f_{i}, \partial_{x} f_{i}\right\rangle+\frac{5}{2}\left\langle\partial_{x}^{2} f_{i}, \partial_{x} f_{i}\right\rangle \frac{\left|\partial_{x}^{2} f_{i}\right|^{2}}{\left|\partial_{x} f_{i}\right|^{7}} \\
& -\frac{35}{2} \frac{\left(\left\langle\partial_{x}^{2} f_{i}, \partial_{x} f_{i}\right\rangle\right)^{3}}{\left|\partial_{x} f_{i}\right|^{9}}+\lambda_{i} \frac{\left\langle\partial_{x}^{2} f_{i}, \partial_{x} f_{i}\right\rangle}{\left|\partial_{x} f_{i}\right|^{3}}
\end{aligned}
$$

we get the parabolic equations

$$
\partial_{t} f_{i}=-\frac{1}{\left|\partial_{x} f_{i}\right|^{4}} \partial_{x}^{4} f_{i}+h\left(f_{i}\right)
$$

for $i=1,2,3$, with

$$
\begin{aligned}
h\left(f_{i}\right)= & 6\left\langle\partial_{x}^{2} f_{i}, \partial_{x} f_{i}\right\rangle \frac{\partial_{x}^{3} f_{i}}{\left|\partial_{x} f_{i}\right|^{6}} \\
& +\frac{\partial_{x}^{2} f_{i}}{\left|\partial_{x} f_{i}\right|^{2}}\left(\frac{5}{2} \frac{\left|\partial_{x}^{2} f_{i}\right|^{2}}{\left|\partial_{x} f_{i}\right|^{4}}+4 \frac{\left\langle\partial_{x}^{3} f_{i}, \partial_{x} f_{i}\right\rangle}{\left|\partial_{x} f_{i}\right|^{4}}-\frac{35}{2} \frac{\left(\left\langle\partial_{x}^{2} f_{i}, \partial_{x} f_{i}\right\rangle\right)^{2}}{\left|\partial_{x} f_{i}\right|^{6}}+\lambda_{i}\right) .
\end{aligned}
$$

The geometric boundary condition $\vec{\kappa}_{i}=0$ is also a source of trouble. To circumvent also this problem we construct a solution to (C2) with boundary conditions

$$
\left\{\begin{array}{rrr}
f_{i}(t, 1) & =P_{i}, & \text { for all } t \in[0, T), i=1,2,3, \\
\partial_{x}^{2} f_{i}(t, 1)=0=\partial_{x}^{2} f_{i}(t, 0) & \text { for all } t \in[0, T), i=1,2,3, \\
f_{1}(t, 0)=f_{2}(t, 0)=f_{3}(t, 0) & \text { for all } t \in[0, T), \\
\text { and } \sum_{i=1}^{3}\left(\nabla_{s} \vec{\kappa}_{i}(t, 0)-\lambda_{i} \partial_{s} f_{i}(t, 0)\right)=0 & \text { for all } t \in[0, T),
\end{array}\right.
$$

Precisely we show in [6] that the following theorem holds.

Theorem C.1. Let $n \geq 2$ and $P_{1}, P_{2}, P_{3}$ be distinct points in $\mathbb{R}^{n}$. Given $f_{i, 0}:[0,1] \rightarrow \mathbb{R}^{n}, f_{i, 0} \in C^{\infty}([0,1])$, $i=1,2,3$, regular maps satisfying the compatibility conditions of any order (as stated in Remark C.3 below) and such that the non-collinearity condition for the unit tangent vectors holds at the triple junction (i.e. (5.1) holds for the initial network at the triple junction), then there exist $T>0$ and regular curves $f_{i} \in C^{\infty}\left([0, T] \times[0,1] ; \mathbb{R}^{n}\right)$, $i=1,2,3$, such that $f=\left(f_{1}, f_{2}, f_{3}\right)$ is the unique solution of (C2) together with the boundary conditions (C4) and the initial condition $f_{i}(t=0)=f_{i, 0}$. Moreover, the non-collinearity condition (5.1) holds at the triple junction for any time $t \in[0, T]$. 
Remark C.2. If the initial datum $\left(f_{1,0}, f_{2,0}, f_{3,0}\right)$ satisfies just $f_{i, 0} \in C^{4, \alpha}([0,1]), i=1,2,3$ and $\alpha \in(0,1)$, and compatibility condition of order zero (i.e. $\mu=0$ in Remark C.3 below) then we prove in [6] existence and uniqueness of a solution of (C2) and (C4) in the parabolic Hölder spaces $C^{\frac{4+\alpha}{4}, 4+\alpha}\left([0, T] \times[0,1] ; \mathbb{R}^{n}\right)$. This solution is also smooth for postive times (i.e. it belongs to $\left.C^{\infty}\left((0, T] \times[0,1] ; \mathbb{R}^{n}\right)\right)$ thanks to the parabolicity of the equation.

In [6] we refer to the solution given in Theorem C.1 as solution of the analytical problem (C2), (C4). Obviously such a solution solves (1.8) together with (1.9).

Remark C.3. (Compatibility conditions analytical problem) Following [23, page 98] and [11, page 217, Example 6.12], for the problem (C2), (C4), with initial datum $f_{0}$, we say that compatibility conditions of order $\mu \in \mathbb{N} \cup\{0\}$ are satisfied if the following hold:

- we have

$$
f_{i, 0}(1)=P_{i}, i=1,2,3 \text { and } f_{i, 0}(0)-f_{j, 0}(0)=0 j \neq i,
$$

- for any $i_{q} \in \mathbb{N}$ such that $4 i_{q}-4 \leq \mu$ we have

$$
\left.\partial_{t}^{i_{q}} f_{i}\right|_{(t, x)=(0,1)}=0, i=1,2,3 \text { and }\left.\left(\partial_{t}^{i_{q}} f_{i}-\partial_{t}^{i_{q}} f_{j}\right)\right|_{(t, x)=(0,0)}=0 j \neq i,
$$

- for any $i_{q} \in \mathbb{N} \cup\{0\}$ such that $4 i_{q}-2 \leq \mu$ we have

$$
\left.\partial_{t}^{i_{q}}\left(\partial_{x}^{2} f_{i}\right)\right|_{(t, x)=(0,1)}=0, \text { and }\left.\partial_{t}^{i_{q}}\left(\partial_{x}^{2} f_{i}\right)\right|_{(t, x)=(0,0)}=0, i=1,2,3
$$

- for any $i_{q} \in \mathbb{N} \cup\{0\}$ such that $4 i_{q}-1 \leq \mu$ we have

$$
\left.\partial_{t}^{i_{q}}\left(\sum_{i=1}^{3}\left(\nabla_{s} \vec{\kappa}_{i}(t, 0)-\lambda_{i} \partial_{s} f_{i}(t, 0)\right)\right)\right|_{t=0}=0 .
$$

The above conditions should to be understood as follows: upon recalling (1.8), (C3), and (C1), let $L_{i}^{*}, i=1,2,3$, be the differential operator such that

$$
\begin{aligned}
L_{i}^{\star} f_{i} & =\partial_{t} f_{i}=-\frac{1}{\left|\partial_{x} f_{i}\right|^{4}} \partial_{x}^{4} f_{i}+h\left(f_{i}\right) \\
& =-\nabla_{s}^{2} \overrightarrow{\kappa_{i}}-\frac{1}{2}\left|\vec{\kappa}_{i}\right|^{2} \vec{\kappa}_{i}+\lambda_{i} \vec{\kappa}_{i}+\varphi_{i}^{\star} \partial_{s} f_{i}
\end{aligned}
$$

and let

$$
L_{i}^{\star\left(i_{q}\right)} f_{i}=\partial_{t}^{i_{q}} f_{i},
$$

where one can use Lemmas 3.1, 3.5, and Lemma 3.6 to derive an explicit expression for $\partial_{t}^{i_{q}} f_{i}$ free of time derivatives. Then the first condition can be rephrased as

$$
\begin{aligned}
& L_{i}^{\star\left(i_{q}\right)} f_{i, 0}=0 \text { at } x=1 \text { for } i=1,2,3, \text { and } \\
& L_{i}^{\star\left(i_{q}\right)} f_{i, 0}=L_{j}^{\star\left(i_{q}\right)} f_{j, 0} \text { at } x=0 \text { for } i \neq j .
\end{aligned}
$$

The other conditions are understood in a similar way. For instance the second set of conditions can be rephrased as

$$
\partial_{x}^{2} L_{i}^{*\left(i_{q}\right)} f_{i, 0}=0 \text { at } x \in\{0,1\} \text { for } i=1,2,3 .
$$

Lemma C.4. Let $f_{i}:[0, T] \times[0,1] \rightarrow \mathbb{R}^{n}, f_{i}=f_{i}(t, x), i=1,2,3$, be a $C^{\infty}([0, T] \times[0,1])$ solution of the system

$$
\partial_{t} f_{i}=-\nabla_{s}^{2} \vec{\kappa}_{i}-\frac{1}{2}\left|\vec{\kappa}_{i}\right|^{2} \vec{\kappa}_{i}+\lambda_{i} \vec{\kappa}_{i}+\varphi_{i}^{I} \partial_{s} f_{i} \quad i=1,2,3
$$


with given initial datum $f_{i}(t=0)=f_{i, 0} \in C^{\infty}([0,1])$ and boundary conditions

$$
\left\{\begin{array}{rlrl}
f_{i}(t, 1) & =P_{i}, & \text { for all } t \in[0, T], i=1,2,3, \\
\partial_{x}^{2} f_{i}(t, 1)=0=\partial_{x}^{2} f_{i}(t, 0) & \text { for all } t \in[0, T], i=1,2,3, \\
f_{1}(t, 0)=f_{2}(t, 0)=f_{3}(t, 0) & \text { for all } t \in[0, T], \\
\text { and } \sum_{i=1}^{3}\left(\nabla_{S} \vec{\kappa}_{i}(t, 0)-\lambda_{i} \partial_{s} f_{i}(t, 0)\right)=0 & \text { for all } t \in[0, T] .
\end{array}\right.
$$

Here $\varphi_{i}^{I} \in C^{\infty}([0, T] \times[0,1]) \rightarrow \mathbb{R}$ are given maps in the sense that

$$
\varphi_{i}^{I}=\varphi_{i}^{I}\left(f_{i}, \partial_{x} f_{i}, \partial_{x}^{2} f_{i}, \partial_{x}^{3} f_{i}, \partial_{x}^{4} f_{i}\right) .
$$

Moreover we assume that $\varphi_{i}^{I}(t, x), x \in\{0,1\}$, is invariant under reparametrization of $f$ (i.e. the tangential component is a geometric term at the boundary).

Let $\psi_{i}:[0,1] \rightarrow[0,1]$ be a smooth diffeomorphism such that $\partial_{x} \psi_{i}>0, i=1,2,3$.

Then there exists a unique family of diffeomorphisms $\phi_{i}(t, \cdot)$ with $\phi_{i} \in C^{\infty}\left(\left[0, T^{\prime}\right] \times[0,1]\right) \rightarrow \mathbb{R}, 0<T^{\prime} \leq T$, such that $\phi_{i}(0, \cdot)=\psi_{i}(\cdot)$ and $\tilde{f}_{i}(t, x):=f\left(t, \phi_{i}(t, x)\right)$ satisfies

$$
\partial_{t} \tilde{f}_{i}=-\nabla_{s}^{2} \overrightarrow{\tilde{\kappa}}_{i}-\frac{1}{2}\left|\overrightarrow{\tilde{\kappa}}_{i}\right|^{2} \overrightarrow{\tilde{\kappa}}_{i}+\lambda_{i} \overrightarrow{\tilde{\kappa}}_{i}+\varphi_{i}^{I I} \partial_{s} \tilde{f}_{i}
$$

with initial datum $\tilde{f}_{i}(t=0)=\tilde{f}_{0, i} \in C^{\infty}([0,1]), i=1,2,3$, and boundary conditions

$$
\left\{\begin{array}{lr}
\tilde{f}_{i}(t, 1)=P_{i}, & \text { for all } t \in\left[0, T^{\prime}\right], i=1,2,3, \\
\vec{\kappa}_{i}(t, 1)=0=\vec{\kappa}_{i}(t, 0) & \text { for all } t \in\left[0, T^{\prime}\right], i=1,2,3, \\
\tilde{f}_{1}(t, 0)=\tilde{f}_{2}(t, 0)=\tilde{f}_{3}(t, 0) & \text { for all } t \in\left[0, T^{\prime}\right], \\
\text { and } \sum_{i=1}^{3}\left(\nabla_{s} \overrightarrow{\tilde{\kappa}}_{i}(t, 0)-\lambda_{i} \partial_{s} \tilde{f}_{i}(t, 0)\right)=0 & \text { for all } t \in\left[0, T^{\prime}\right] .
\end{array}\right.
$$

Here $\overrightarrow{\tilde{\kappa}}$ is the curvature vector of the curve $\tilde{f}$ and $\varphi_{i}^{I I}$ is given by

$$
\begin{aligned}
\varphi_{i}^{I I}(t, x) & =\varphi_{i}^{I}(t, 0)\left(1-\frac{1}{\mathcal{L}\left(\tilde{f}_{i}(t)\right)} \int_{0}^{x}\left|\partial_{x} \tilde{f}_{i}(t, \xi)\right| d \xi\right) \\
& =\varphi_{i}^{I}(t, 0)\left(1-\frac{1}{\mathcal{L}\left(f_{i}(t)\right)} \int_{0}^{x}\left|\partial_{x} f_{i}\left(t, \phi_{i}(t, \xi)\right) \partial_{x} \phi_{x}(t, \xi)\right| d \xi\right) .
\end{aligned}
$$

Note that, like $\varphi_{i}^{I}$, also $\varphi_{i}^{I I}$ is a geometric term at the boundary (by the assumptions on $\varphi_{i}^{I}$ ). In our applications where $\varphi_{i}^{I}=\varphi_{i}^{\star}$ as given in (C1), we see upon recalling Remark 5.1 that $\varphi_{i}^{I I}$ at the boundary can be also expressed via (C1) where $f_{i}$ is there replaced by $\tilde{f}_{i}$.

Proof. First of all let us derive the system that $\phi_{i}, i=1,2,3$, must satisfy. Since for each time $\phi_{i}(t, \cdot)$ must be a diffeomorphism of the interval $[0,1]$ into itself, we ask that

$$
\phi_{i}(t, 0)=0, \quad \phi_{i}(t, 1)=1 \quad \text { for all } t .
$$

Since $\tilde{f}_{i}(t, x)=f_{i}\left(t, \phi_{i}(t, x)\right)$ we obviously have

$$
\partial_{x} \tilde{f}_{i}(t, x)=\partial_{x} f\left(t, \phi_{i}(t, x)\right) \partial_{x} \phi_{i}(t, x),
$$

hence provided

$$
\partial_{x} \phi_{i}(t, x)>0 \text { for all }(t, x)
$$


then the tangent vectors of $f_{i}$ and $\tilde{f}_{i}$ coincide, that is $\partial_{s} \tilde{f}_{i}(t, x)=\partial_{s} f_{i}\left(t, \phi_{i}(t, x)\right)$. The same holds for all geometric quantities since they are invariant under reparametrization, that is $\overrightarrow{\tilde{\kappa}}_{i}(t, x)=\vec{\kappa}_{i}(t, \phi(t, x))$ and so on. Next observe that

$$
\partial_{x}^{2} \tilde{f}_{i}(t, x)=\partial_{x}^{2} f_{i}\left(t, \phi_{i}(t, x)\right)\left(\partial_{x} \phi_{i}(t, x)\right)^{2}+\partial_{x} f_{i}\left(t, \phi_{i}(t, x)\right) \partial_{x}^{2} \phi_{i}(t, x),
$$

In particular by (C9) and (C6) it follows that at the boundary $x \in\{0,1\}$ we have

$$
\partial_{x}^{2} \tilde{f}_{i}(t, x)=\partial_{x}^{2} f_{i}(t, x)\left(\partial_{x} \phi_{i}(t, x)\right)^{2}+\partial_{x} f_{i}(t, x) \partial_{x}^{2} \phi_{i}(t, x)=\partial_{x} f_{i}(t, x) \partial_{x}^{2} \phi_{i}(t, x),
$$

and hence $\overrightarrow{\tilde{\kappa}}=0$ at the boundary. In fact if $\psi_{i}(x)=x$ the above computation even yields that $\partial_{x}^{2} \tilde{f}_{i}(t=0, x)=0$ for $x \in\{0,1\}$. The other boundary conditions are satisfied since they involve pure geometric quantities. Finally notice that using the flow equation

$$
\begin{aligned}
\partial_{t} \tilde{f}_{i}(t, x)= & \partial_{t} f_{i}\left(t, \phi_{i}(t, x)\right)+\partial_{x} f_{i}\left(t, \phi_{i}(t, x)\right) \partial_{t} \phi_{i}(t, x) \\
= & {\left[-\nabla_{s}^{2} \vec{\kappa}_{i}-\frac{1}{2}\left|\vec{\kappa}_{i}\right|^{2} \vec{\kappa}_{i}+\lambda_{i} \vec{\kappa}_{i}+\varphi_{i}^{I} \partial_{s} f_{i}\right]\left(t, \phi_{i}(t, x)\right)+\partial_{x} f_{i}\left(t, \phi_{i}(t, x)\right) \partial_{t} \phi_{i}(t, x) } \\
= & {\left[-\nabla_{s}^{2} \overrightarrow{\tilde{\kappa}}_{i}-\frac{1}{2}\left|\overrightarrow{\tilde{\kappa}}_{i}\right|^{2} \overrightarrow{\tilde{\kappa}}_{i}+\lambda_{i} \overrightarrow{\tilde{\kappa}}_{i}\right](t, x) } \\
& +\left[\varphi_{i}^{I}\left(t, \phi_{i}(t, x)\right)+\left|\partial_{x} f_{i}\left(t, \phi_{i}(t, x)\right)\right| \partial_{t} \phi_{i}(t, x)\right] \partial_{s} f_{i}\left(t, \phi_{i}(t, x)\right)
\end{aligned}
$$

and hence

$$
\begin{aligned}
\partial_{t} \tilde{f}_{i}(t, x)= & {\left[-\nabla_{s}^{2} \overrightarrow{\tilde{\kappa}}_{i}-\frac{1}{2}\left|\overrightarrow{\tilde{\kappa}}_{i}\right|^{2} \overrightarrow{\tilde{\kappa}}_{i}+\lambda_{i} \overrightarrow{\tilde{\kappa}}_{i}+\varphi_{i}^{I I} \partial_{s} \tilde{f}_{i}\right](t, x) } \\
& +\left[\varphi_{i}^{I}\left(t, \phi_{i}(t, x)\right)-\varphi_{i}^{I I}(t, x)+\left|\partial_{x} f_{i}\left(t, \phi_{i}(t, x)\right)\right| \partial_{t} \phi_{i}(t, x)\right] \partial_{s} f_{i}\left(t, \phi_{i}(t, x)\right) .
\end{aligned}
$$

It follows that the diffeomorphism $\phi_{i}$ has to solve the first order ODE

$$
\partial_{t} \phi_{i}(t, x)=\frac{1}{\left|\partial_{x} f_{i}\left(t, \phi_{i}(t, x)\right)\right|}\left(-\varphi_{i}^{I}\left(t, \phi_{i}(t, x)\right)+\varphi_{i}^{I I}(t, x)\right),
$$

with initial datum $\phi_{i}(0, x)=\psi(x)$ for each $x \in[0,1]$. The right hand side in (C12) can be written as $G\left(t, x, \phi_{i}(t, x)\right)$, with $G(t, x, y)$ a smooth functions in its variables. Here $x$ plays the role of a parameter, $x \in[0,1]$. Since $\varphi_{i}^{I}(t, x)=\varphi_{i}^{I I}(t, x)$ for $x \in\{0,1\}$ and all $t$, we see that the solution at $x=0,1$ is $\phi_{i}(t, x)=x$ for all $t$, i.e. (C9) is satisfied. The existence, uniqueness and regularity of the solution follow from [19, Sec.1.3] and [16, Chap.9 and App.D]. The smoothness of the solution together with the assumption on the initial datum $\partial_{x} \psi_{i}>0$ imply that also (C10) is satisfied.

\section{References}

[1] Barrett, J. W., Garcke, H., and Nürnberg, R. Elastic flow with junctions: variational approximation and applications to nonlinear splines. Math. Models Methods Appl. Sci. 22, 11 (2012).

[2] Bronsard, L., and Reitich, F. On three-phase boundary motion and the singular limit of a vector-valued Ginzburg-Landau equation. Arch. Rational Mech. Anal. 124, 4 (1993), 355-379.

[3] Buxton, G. A., and Clarke, N. "Bending to stretching" transition in disordered networks. Phys. Rev. Lett. 98 (2007), 238103.

[4] Dall'Acqua, A., Lin, C.-C., and Pozzi, P. Evolution of open elastic curves in $\mathbb{R}^{n}$ subject to fixed length and natural boundary conditions. Analysis (Berlin) 34, 2 (2014), 209-222.

[5] Dall'Acqua, A., Lin, C.-C., and Pozzi, P. A gradient flow for open elastic curves with fixed length and clamped ends. Ann. Sc. Norm. Super. Pisa Cl. Sci. (5) 17, 3 (2017), 1031-1066.

[6] Dall'Acqua, A., Lin, C.-C., and Pozzi, P. Elastic flow of networks: short-time existence result. Preprint 2019 (2019).

[7] Dall'Acqua, A., Novaga, M., and Pluda, A. Minimal elastic networks. accepted in Indiana University Mathematics Journal (2017).

[8] Dall'Acqua, A., and Pozzi, P. A Willmore-Helfrich $L^{2}$-flow of curves with natural boundary conditions. Comm. Anal. Geom. 22, 4 (2014), 617-669. 
[9] Dall'Acqua, A., and Pozzi, P. On a Willmore-Helfrich $L^{2}$-flow of open curves in $\mathbb{R}^{2}$ : a different approach. RIMS Kokyuroku 1974 (2015), 68-82.

[10] Dziuk, G., Kuwert, E., and Schätzle, R. Evolution of elastic curves in $\mathbb{R}^{n}$ : existence and computation. SIAM J. Math. Anal. 33, 5 (2002), 1228-1245 (electronic).

[11] Eidelman, S. D., and Zhitarashu, N. V. Parabolic boundary value problems, vol. 101 of Operator Theory: Advances and Applications. Birkhäuser Verlag, Basel, 1998. Translated from the Russian original by Gennady Pasechnik and Andrei lacob.

[12] Garcke, H., Menzel, J., and Pluda, A. Long time existence of solutions to an elastic flow of networks. Preprint 2019 (https://arxiv.org/abs/1901.03246).

[13] Garcke, H., Menzel, J., and Pluda, A. Willmore flow of planar networks. J. Differential Equations 266, 4 (2019), $2019-2051$.

[14] Gurtin, M. Thermomechanics of evolving phase boundaries in the plane. Oxford Science Publication, 1993.

[15] Gurtner, G., and Durand, M. Stiffest elastic networks. Proc. R. Soc. A 470 (2014), 20130611.

[16] Lee, J. M. Introduction to smooth manifolds, second ed., vol. 218 of Graduate Texts in Mathematics. Springer, New York, 2013.

[17] Lin, C.-C. $L^{2}$-flow of elastic curves with clamped boundary conditions. J. Differential Equations 252, 12 (2012), $6414-6428$.

[18] Lowengrub, J. S., Rätz, A., and Voigt, A. Phase-field modeling of the dynamics of multicomponent vesicles: spinodal decomposition, coarsening, budding, and fission. Phys. Rev. E 79, 3 (2009), 0311926, 13 pp.

[19] Mantegazza, C. Lecture Notes on Mean Curvature Flow, vol. 290 of Progress in Mathematics. Birkhäuser Basel, 2011.

[20] Mantegazza, C., Novaga, M., Pluda, A., and Schulze, F. Evolution of networks with multiple junctions. preprint (2016).

[21] Novaga, M., and Pozzi, P. A second order gradient flow of p-elastic planar networks. Preprint 2019 (https://arxiv.org/abs/1905.06742) (2019).

[22] Polden, A. Curves and surfaces of least total curvature and fourth-order flows. PhD Thesis, Universität Tübingen (1996).

[23] Solonnikov, V. A. Boundary Value Problems of Mathematical Physics. III. No. 83 in Proceedings of the Steklov institute of Mathematics (1965). Amer. Math. Soc., Providence, R. I., 1967. 